\begin{tabular}{|c|l|}
\hline Title & A mathematical mechanism for instabilities in stripe formation on growing domains \\
\hline Author(s) & Ueda, Kei-Ichi; Nishiura, Y asumasa \\
\hline Citation & $\begin{array}{l}\text { Physica D : Nonlinear Phenomena, 241(1), 37-59 } \\
\text { https://doi.org/10.1016/.physd.2011.09.016 }\end{array}$ \\
\hline Issue Date & 2012-01-01 \\
\hline Doc URL & http://hdl.handle.net/2115/48153 \\
\hline Type & article (author version) \\
\hline File Information & PhyA 241-1_37-59.pdf \\
\hline
\end{tabular}

Instructions for use 


\title{
A mathematical mechanism for instabilities in stripe formation on growing domains
}

\author{
Kei-Ichi Ueda ${ }^{\mathrm{a}}$, Yasumasa Nishiura ${ }^{\mathrm{b}}$ \\ ${ }^{a}$ Research Institute for Mathematical Sciences, Kyoto University, \\ Sakyo-ku, Kyoto 606-8502, Japan. \\ ${ }^{b}$ Research Institute for Electronic Science, Hokkaido University, \\ Kita-ku, Sapporo 001-0020, Japan.
}

\begin{abstract}
A cascade process involving stripe splitting in reaction-diffusion systems with isotropically growing one-dimensional domains is studied. Such cascades, propagating from a smaller domain to a larger domain, have been proposed as an answer to the criticism that the Turing mechanism lacks robustness because many stable patterns can coexist on a large domain and, therefore, the final state is very sensitive to the initial conditions. In contrast, if the system starts with a small domain, very few stable patterns are possible, which limits the sensitivity to the initial conditions. In order to show the validity and limitations of this scenario, we clarify the underlying mathematical mechanism that drives various types of stripe-splitting via a reduction from partial differential equations to ordinary differential equations, as well as investigating global arrangements of the set of $n$-mode stripe branches with $D_{n}$-symmetry of the stripe locations. The mathematical simplification above allows us to reveal how each $n$-mode stripe branch is destabilized as the domain grows and to characterize the associated eigenprofiles that actually determine the manner of splitting at the infinitesimal level. We find that all the $D_{n}$-symmetry-breaking instabilities typically occur simultaneously up to leading order before the saddle-node point of the $n$-mode stripe branch is reached. The instability with the largest real part is of the alternate type: every other peak splits at the infinitesimal level. A symmetry-preserving instability appears at the saddle-node point, which drives the simultaneous type of splitting, i.e., mode-doubling. Due to competition between these two types of instabilities, the problem depends subtly on the growth speed. Alternate splitting typically arises for slow growth and simultaneous splitting for fast growth. For intermediate growth rates, the manner of splitting becomes mixed and sensitive to fluctuations.
\end{abstract}

Keywords: Stripe splitting, Growing domain, Reaction-diffusion system 2000 MSC: 35B 32, 35K57, 34K 17

\section{Introduction}

The formation of patterns on growing domains is one of the key issues in developmental biology, and self-organized dynamics combined with genetic control is believed to form the core part of the underlying mechanism. For instance, the pigment patterns that are present on growing fish skin [1] represent a remarkable example of the Turing mechanism [2] that can be observed at a macroscopic level; the manner in which stripe splitting and rearrangement occurs 
has attracted much attention $[3,4,5,6]$. Stripe splitting on a growing domain corresponds to a take-off and landing from one stripe branch to another in solution space. The driving force for this process may originate from instabilities of the solution branches and their global bifurcation structures. In addition to these factors, the most subtle issue to be considered involves the fact that the manner of splitting strongly depends on the speed of domain growth. This is because the strength and type of instability for take-off and landing varies with the speed of growth, hence it is not a priori clear which route the orbit will select among the many possible cascades for a given targeted size of domain. Hereafter, we refer to a $n$-mode stripe pattern (solution) as simply a $n$-stripe pattern (solution).

In this paper we study the cascade processes involved in stripe (pulse) splitting for the reaction-diffusion system described by (1) in isotropically growing 1D domains. Such cascade dynamics have a potential to produce robust dynamics against the selection of initial data and parameters, however the dependency on the growing speed of its dynamics remains open so far. One of our goals is to clarify the relation between the instability mechanism of each stripe and the growing speed. As a domain grows, the solution hops several times from one branch to another, and eventually arrives at a more or less uniquely determined final pattern for the targeted domain. There is a considerable body of literature on this topic. For instance, Crampin et al. [7] showed that a mode-doubling sequence occurs robustly on an exponentially growing domain, i.e., all the stripes split simultaneously and hence their number increases proportionally to $2^{n}$. Growth-function-dependent pattern formation has been studied $[8,9,10]$ and the interaction between domain growth and pattern formation has also been discussed [11]. In addition, biological patterns have been reproduced on growing domains [12, 13, 14]. However, when the speed of growth is sufficiently low, this mode-doubling splitting sequence breaks down and more complex sequences are observed [7]. Figure 1 shows typical splitting sequences on linearly and isotropically growing domains with different growth speeds, based on the Gray-Scott model [15]:

$$
\left\{\begin{array}{l}
\frac{\partial u}{\partial t}=D_{u} u_{x x}-u v^{2}+A(1-u), \\
\frac{\partial v}{\partial t}=D_{v} v_{x x}+u v^{2}-(A+k) v, \quad x \in\left[0, L_{0} \gamma(t)\right], \quad t>0 . \\
\frac{d \gamma}{d t}=c
\end{array}\right.
$$

Here, $L_{0}$ is the initial size of the domain, $c$ is the growth rate, and $L_{0} \gamma(t)$ is the size of the domain at time $t$. It is known that the Gray-Scott model (1) yields a variety of dynamic patterns including self-replication, as well as the usual pulses and spots on fixed domains [16]. The derivation of the model system (1) and its normalized version on unit interval $[0,1]$ is given in Section 2. Three different types of splitting are depicted in Fig. 1. Such a delicate dependence on the growth rate is observed not only for our model system, but also for a large class of other reaction-diffusion systems, as discussed in Section 6. We note that asymmetric spot splitting sequences have been observed on a growing domain in two-dimensional space [17]. Barrass et al. [18] have clarified how the breakdown of the mode-doubling sequence occurs by computing a global bifurcation diagram for stripe solutions. However, in the work of [18], as well as in the other literature cited above, no analysis of the alternate splitting that is typically observed on slowly growing domains has been carried out, and the general mechanism of symmetry-breaking splitting dynamics, including the alternate splitting process, remains an open problem. 


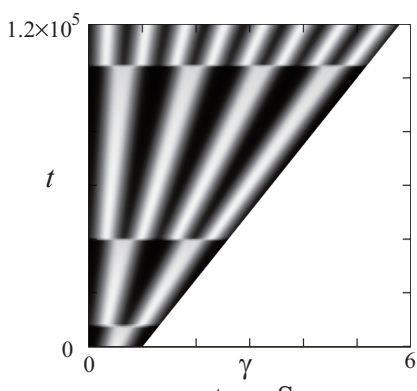

type-S

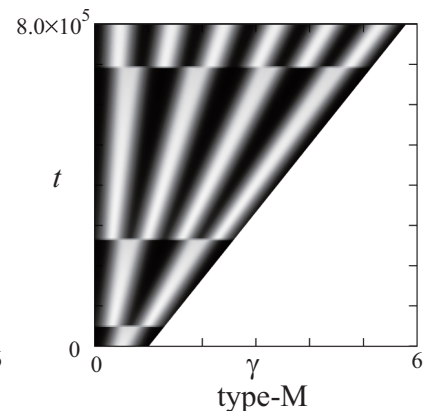

(a)

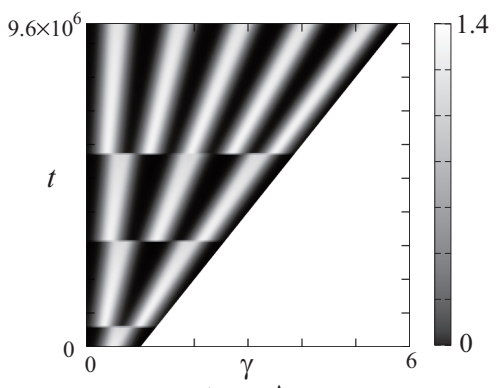

type-A
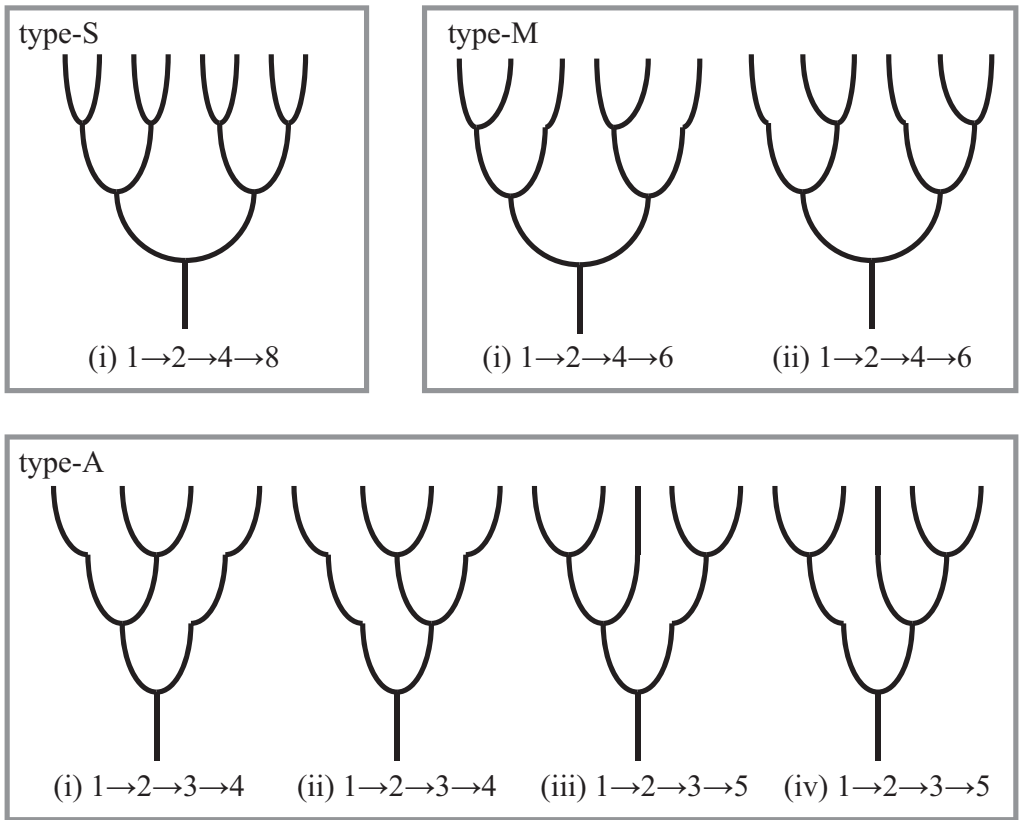

(b)

Figure 1: Three different types of splitting sequences are observed in (1). Type-S: All of the stripes split simultaneously at the second and the third splitting. Type-A: Every other peak splits at the second and the third splitting. Type-M; Mixed splitting, i.e., all of the stripes split at the second splitting and every other peak splits at the third splitting. Type-S and type-A are typically observed for fast and slow growth, respectively, and Type-M for intermediate cases. (a) Typical splitting sequences on linearly growing domains: type-S (growth rate $\left.c=4.0 \times 10^{-5}\right)$, type-M $\left(c=6.0 \times 10^{-6}\right)$ and typeA $\left(c=5.0 \times 10^{-7}\right)$. In this plot and hereafter, the profile of the $v$-component is displayed unless otherwise stated. (b) Schematic depiction of splitting sequences. Here, the parameters of (1) were set to $A=0.22, k=0.041, D_{u}=2.0 \times 10^{3}$, $D_{v}=1.0 \times 10^{2}$, and $L_{0}=400$. 
It should be emphasized that it is quite difficult to predict the behavior of solutions based only on information concerning each fixed domain size, as illustrated by Fig.1. This is because the splitting process also depends heavily on the growth speed. Nevertheless, it is of fundamental importance to understand the existence and stability properties of $n$-stripe solutions for a given fixed domain size. Indeed, the manner of the splitting (at least at the onset of the process) is closely linked to the eigenprofile of the eigenvalue with the largest real part, as discussed in Section 4. The main goal of our current study is to show analytically the type of instability that exists as the domain grows for $n$-stripe solutions under Neumann or periodic boundary conditions. More precisely, we derive finite-dimensional ordinary differential equations (ODEs) for well-separated stripes near the saddle-node point and we study their stabilities, taking the minimum distance as a bifurcation parameter. We find that $D_{n}$-symmetry-breaking instabilities occur simultaneously up to leading order on the $n$-stripe branch, followed by a saddle-node bifurcation as the domain grows. The eigenprofile associated with the eigenvalue of the largest real part alternates in sign. Therefore, every second hump starts to split at the infinitesimal level for each fixed domain size. The eigenfunction at the saddle-node point, which is $D_{n}$-symmetry preserving, triggers simultaneous splitting: all humps start to split when the domain size is larger than the position of the saddle-node point. It should be noted that these results are model-independent because all information arising from the nonlinearity is embedded in the coefficients of the resulting ODEs (see Section 6.3). We confirm these conclusions numerically for the first few stripe branches of both our model system (1) and an equivalent normalized system (6) (see Section 2). The above results explain a number of important observations. For instance, if the growth rate is very slow then the alternate splitting process can initiate instability and eventually dominate the system. In contrast, when the growth is rapid (for example with an exponential rate), the instability of the simultaneous splitting associated with the saddle-node point dominates the system. Noting that all the unstable symmetry-breaking eigenfunctions are orthogonal to the $n$-stripe solution with $D_{n}$-symmetry, it is easily seen that small fluctuations deviating from the $P_{n}$-branch do not grow sufficiently before reaching the saddle-node point when the growth is appropriately fast, such as for type-S growth in Fig. 1. Here, a delicate balance between the different types of instability, growth rate, and external fluctuations is clearly observed. If the above factors are of comparable importance, the resulting dynamics becomes much more complicated; in other words, the winner at the infinitesimal level does not necessarily dominate the dynamics as a whole, and a tiny fluctuation can change the fate of the pattern completely. That is, although pattern formation on growing domains is much more robust than usual Turing mechanism, there is a subtle regime in which it is difficult to control and predict its dynamics. We discuss this issue further in Section 6.

Although we focus on the case of weak interactions in this paper, there are many studies on the existence, stability and pulse-splitting behavior of spike patterns on fixed domains that depend on the interaction strength and the feeding rate. For instance, there is an important class of systems in the semi-strong regime, which has been extensively studied by Doelman, Kaper, and their collaborators $[19,20,21,22,23,24]$; another class is comprised of systems in the pulse-splitting regime, investigated primarily by Kolokolnikov, Ward, and Wei $[25,26]$ (see also $[27,28,29,30])$. While the works cited above do not study domain growth problems specifically, they will play a fundamental role in the extension of growth problems to these other regimes.

This paper is organized as follows. We introduce our model system, the Gray-Scott model, in the next section. In Section 3, we demonstrate the existence of a parameter regime in which splitting does not occur for any large domain. This is related to the process of pulse-splitting on a line [19, 31, 32, 33] (see also [34]); the saddle-node singularity of the single-pulse branch is the 
point of onset of the splitting. In Section 4, we numerically study the global bifurcation structure of stripe patterns, where domain size is the bifurcation parameter. In particular, we focus on how the symmetry-breaking instabilities occur on the $n$-stripe branch, as well as locating the saddle-node points. We also compute various types of stripe-splitting associated with these instabilities, including a transition from simultaneous to alternate splitting. In Section 5, we derive a finite-dimensional system for a general $n$-stripe pattern from our system of partial differential equations (PDEs). We find that the reduced system inherits most of the essential aspects of the PDE dynamics, and is consistent with the observations made in Section 4. We also show that the eigenmode with the largest real part is of the alternate type. We discuss several issues arising from our study in Section 6, including the response to external perturbations and the robustness of the splitting.

\section{Model system}

We will derive our model system (1) for a growing domain along the lines proposed by Crampin et al. [7]. The analysis and results that we obtain are not restricted to the Gray-Scott model, and are in fact applicable to a much wider class of equations (see Section 6.3 for details).

The derivation of our model system begins with the conservation law

$$
\frac{d}{d t} \int_{0}^{L(t)} \boldsymbol{u}(x, t) d x=\int_{0}^{L(t)}\left[-\frac{\partial}{\partial x} \boldsymbol{j}+F(\boldsymbol{u})\right] d x, \quad x \in[0, L(t)], \quad t>0,
$$

where $L(t): \mathbb{R} \rightarrow \mathbb{R}$ is the domain length at time $t, \boldsymbol{u}={ }^{t}(u(x, t), v(x, t))$ and $F(\boldsymbol{u})={ }^{t}\left(-u v^{2}+\right.$ $\left.A(1-u), u v^{2}-(A+k) v\right)$ are reaction terms, and $\boldsymbol{j}=-D \partial \boldsymbol{u} / \partial x$ is the flux, where $D=\operatorname{diag}\left(D_{u}, D_{v}\right)$ is a diagonal matrix. By using the Reynolds transport theorem, the left-hand side of (2) can be written as

$$
\frac{d}{d t} \int_{0}^{L(t)} \boldsymbol{u}(x, t) d x=\int_{0}^{L(t)}\left[\frac{\partial \boldsymbol{u}}{\partial t}+\frac{\partial}{\partial x}(\rho \boldsymbol{u})\right] d x,
$$

where $\rho(x, t)$ is the flow due to local growth. From (2) and (3), we have

$$
\frac{\partial \boldsymbol{u}}{\partial t}+\frac{\partial}{\partial x}(\rho \boldsymbol{u})=D \frac{\partial^{2} \boldsymbol{u}}{\partial x^{2}}+F(\boldsymbol{u})
$$

In this article, we consider isotropic growth. The flow is determined by

$$
\rho(x, t)=x \frac{\dot{\gamma}(t)}{\gamma(t)}
$$

where the dot denotes the derivative with respect to $t$, and the domain length $L(t)=L_{0} \gamma(t)$ with $\gamma(0)=1$. The derivation of (4) is given in [7].

On transforming to the unit interval while keeping the same notation $(x, t)$,

$$
(x, t) \rightarrow\left(\frac{x}{L(t)}, t\right)
$$


and we obtain

$$
\left\{\begin{array}{l}
\frac{\partial u}{\partial t}=\frac{D_{u}}{\left(L_{0} \gamma(t)\right)^{2}} u_{x x}-u v^{2}+A(1-u)-u \frac{\dot{\gamma}}{\gamma}, \\
\frac{\partial v}{\partial t}=\frac{D_{v}}{\left(L_{0} \gamma(t)\right)^{2}} v_{x x}+u v^{2}-(A+k) v-v \frac{\dot{\gamma}}{\gamma}, \quad x \in[0,1], \quad t>0, \\
\frac{d \gamma}{d t}=\Gamma(t)
\end{array}\right.
$$

where we used the following transformation

$$
\frac{\partial u}{\partial t} \rightarrow \frac{\partial u}{\partial t}+\rho \frac{\partial u}{\partial x}
$$

In the case of linear growth, i.e., $\Gamma(t)=c(c>0)$, we have

$$
\gamma(t)=1+c t
$$

and for exponential growth, i.e., $\Gamma(t)=c^{\prime} \gamma(t)\left(c^{\prime}>0\right)$,

$$
\gamma(t)=e^{c^{\prime} t}
$$

The exponential growth function has been used a lot in previous works [7, 10, 17]. In this paper we mainly focus on the linear growth case which is suitable to see the effect of growth speed on splitting dynamics.

Because $\dot{\gamma}=c^{\prime} \gamma$ holds for exponential growth, the dilution terms $u \dot{\gamma} / \gamma$ and $v \dot{\gamma} / \gamma$ become $c^{\prime} u$ and $c^{\prime} v$, respectively. Therefore, these terms can be incorporated into the kinetics part. In the case of linear growth, the dilution terms depend on $t$ and may affect the evolution of the system; however, they do not play an important role in our setting. This is because, firstly, we consider slow growth with $\dot{\gamma} / \gamma$ in the range from $O\left(10^{-6}\right)$ to $O\left(10^{-5}\right)$, and $0 \leq u, v<2$. Secondly, when $u$ is a solution on the $n$-mode branch, the dilution terms are orthogonal to the instability directions. That is, $\left\langle u \dot{\gamma} / \gamma, \chi^{*}\right\rangle_{L^{2}}=\dot{\gamma} / \gamma\left\langle u, \chi^{*}\right\rangle_{L^{2}}=0$ for any symmetry-breaking eigenfunction $\chi^{*}$ on the $n$ mode branch, and hence the dilution terms have little influence over the orbital selection among the cascades of routes (see Remark 1 in Section 4). Indeed, it is confirmed that numerical results are qualitatively the same whether or not these terms are included in the model systems, even for delicate case that $\dot{\gamma}$ is relatively large as shown in type-S in Fig.1.

Therefore, in this article we employ the following model system for analyzing the splitting phenomena on growing domains:

$$
\left\{\begin{array}{l}
\frac{\partial u}{\partial t}=\frac{D_{u}}{\left(L_{0} \gamma(t)\right)^{2}} u_{x x}-u v^{2}+A(1-u), \\
\frac{\partial v}{\partial t}=\frac{D_{v}}{\left(L_{0} \gamma(t)\right)^{2}} v_{x x}+u v^{2}-(A+k) v, \quad x \in[0,1], \quad t>0 \\
\frac{d \gamma}{d t}=\Gamma(t)
\end{array}\right.
$$


The system (6) can be written as a two-component reaction-diffusion system with time-dependent diffusion coefficients [7]. For example, the diffusion terms of $u$ and $v$-component for linear growth become $D_{u} / L_{0}^{2}(1+c t)^{2}$ and $D_{v} / L_{0}^{2}(1+c t)^{2}$, respectively. The parameters in (6) were set to $A=0.22, D_{u}=2.0 \times 10^{3}, D_{v}=1.0 \times 10^{2}$, and $L_{0}=400$. The constant $k$ is a control parameter. The time step was set to $\Delta t=0.1$ and the spatial mesh size was $\Delta x=2.0 \times 10^{-3}$. A semi-implicit scheme was used for the time integrations, and the Neumann boundary condition was imposed. We note that numerical results similar to those shown in Section 3 and 4 can be observed also for periodic boundary condition.

In the subsequent numerical simulations, except for those involving path-tracking in Section 4, noise is added to both $u$ and $v$ at each iteration in order to avoid artifacts arising from the numerical schemes. The amplitude of the noise was uniformly distributed between $-0.5 \times 10^{-4}$ and $0.5 \times 10^{-4}$, which is sufficiently smaller than the parameters $A$ and $k$ in the kinetics of (6) and larger than the dilution terms. The noise was generated by the minimal random number generator of Park and Miller with Bays-Durham shuffle and added safeguards [35].

\section{Absence of stripe splitting}

It is instructive to consider cases where a stripe does not split, even when the domain size becomes arbitrarily large. As is well known [19, 16, 25, 31, 36], pulse (or spot) splitting can be observed in the extended system in the absence of domain growth, a phenomenon that is often referred to as the self-replication of patterns (Fig.2). It has been shown numerically that a saddlenode bifurcation for a single stripe on the whole line is responsible for this type of splitting [31].

A bifurcation diagram of a 1-stripe solution with respect to $k$ that was obtained numerically under the Neumann boundary conditions is shown in Fig.3(a). Although this diagram was obtained for a finite but large system size, a qualitatively similar diagram can be obtained for the extended case. When the domain length is $L$, the value of the saddle-node point $k_{c}(L)$ for the 1-stripe solution under Neumann or periodic boundary conditions is a monotonically increasing function of $L$ when $L$ is sufficiently large [32]. Let us denote the location of the saddle-node point for an infinite domain by $k=k_{c}$. It is numerically confirmed that $k_{c} \approx 0.046176$. The criterion for the occurrence of splitting in an extended system is that it occurs (does not occur) when $k$ is smaller (larger) than $k_{c}([25,31,32])$. In other words, when $k$ is larger than $k_{c}$, a stable stripe exists for any large domain size and it converges to the homoclinic stripe solution for the same $k$ as the domain size tends to infinity (see the caption of Fig. 3 for the mechanism of the stripe-splitting). Therefore, a necessary condition for the occurrence of stripe-splitting on growing domains is that $k$ should be smaller than $k_{c}$. In fact, if we take $k=0.048>k_{c}$ for our model system, then no splitting occurs for the 1-stripe solution for any large domain as shown in Fig.4. On the other hand, when $k<k_{c}$ is appropriately chosen and the size of the domain is small, there exits a stable branch of 1-stripe, but it can be destabilized as domain grows. The issue in the following sections is to study when and how each stripe is destabilized on growing domains.

\section{Global bifurcation diagram and linearized criterion}

In this section we consider how stripe-splitting is driven by the global arrangement of branches of all relevant stripe patterns and by their stability properties for each fixed size of domain under Neumann boundary conditions. In the following treatment, $P_{n}$ denotes the stationary $n$-mode 


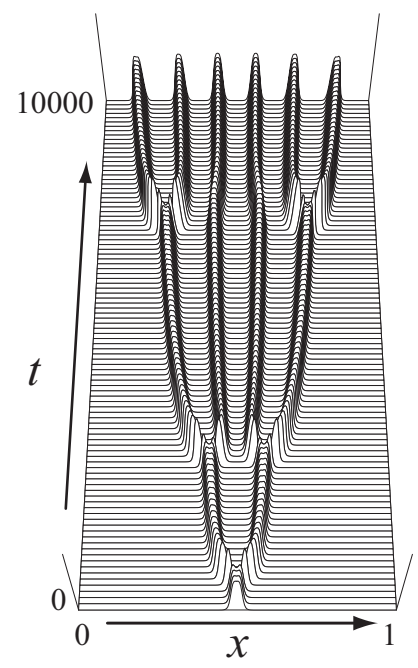

Figure 2: Self-replicating dynamics of (6) with $k=0.046$. The parameter $L$ is fixed to be $3.0 \times 10^{3}$.

branch in the product space $\{\gamma \in \mathbb{R}\} \times L^{2}(0,1)$. A global viewpoint is indispensable in order to predict the manner of splitting. For instance, suppose that the $n$-stripe pattern is destabilized and that its leading linearized spectrum indicates a deformation from the $n$-stripe pattern to a $m$-stripe pattern $(n<m)$. If there is no stable part of the $m$-stripe branch for the associated domain size, then this transition does not occur even though the orbit starts to deform infinitesimally in that direction. As will be shown shortly, the solution profile is almost orthogonal to the unstable eigenspace associated with the largest eigenvalue, so the component projected onto this eigenspace is very small. Therefore, it takes a rather long time for such a disturbance to grow, during which period the domain size changes by a finite amount; another instability, arising from a saddle-node bifurcation, may meanwhile appear in a non-orthogonal direction and eventually drive the orbit in a different direction. A more detailed discussion from the viewpoint of bifurcation with $D_{n}$-symmetry will be given in the next section. Information about the location of all the stripe patterns, their linearized stabilities, and the connection properties of their unstable manifolds for each fixed size of domain is essential for understanding the dynamics on growing domains. However, because of the growth effect, this information is not sufficient to predict the behavior of the orbit, even if the initial data are known precisely. There is subtle competition between the unstable modes and the growth speed before the targeted domain size is reached, and the local winner in space and time is not always the final winner.

\subsection{Geometric arrangement and stability of multi-stripe branches with respect to domain size}

Here, we will numerically investigate the geometric arrangement and stability of multi-stripe patterns using (6), taking the domain size $\gamma$ as the bifurcation parameter. As mentioned in Section 3 , in order to observe splitting on a finite domain under Neumann or periodic boundary conditions, it is necessary that $k<k_{c}$. Let $\tilde{k}_{c}(\gamma)$ be the value of the saddle-node point for the 1-stripe solution when $L=L_{0} \gamma$. In the case of finite domain size, $\tilde{k}_{c}(\gamma)$ is a monotonically increasing function of $\gamma$ when $\gamma$ is sufficiently large [32]. In this section, we assume that $\tilde{k}_{c}(\gamma(0))<k<k_{c}$ 


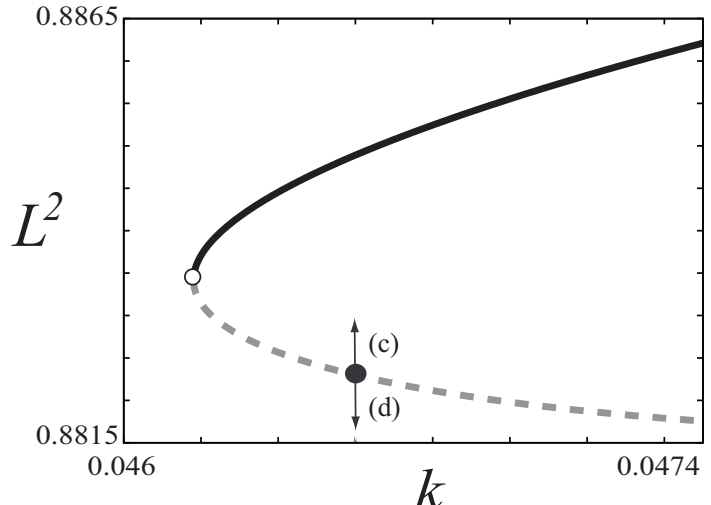

(a)

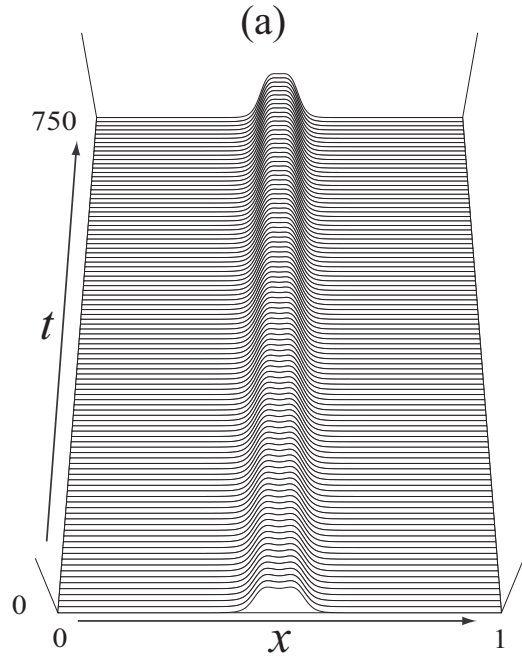

(c)
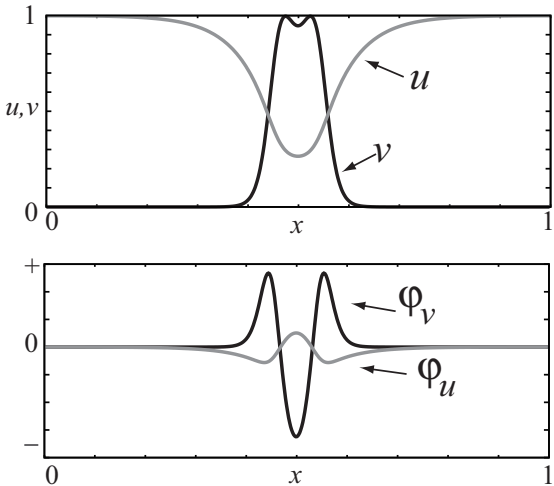

(b)

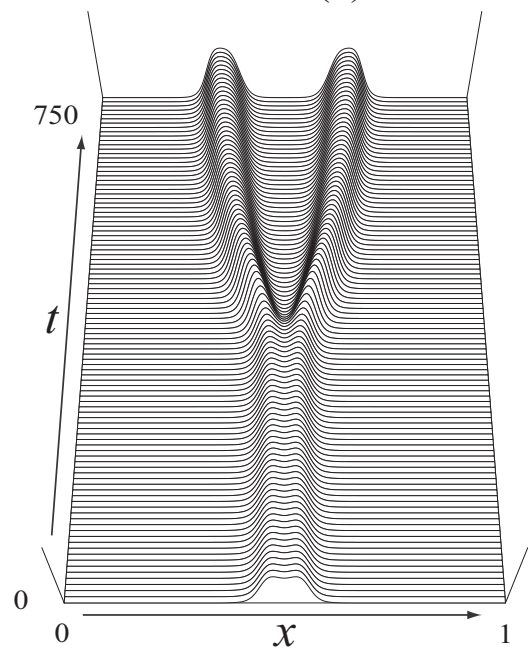

(d)

Figure 3: (a) Bifurcation diagram of a stationary single-stripe solution for $L=1.5 \times 10^{3}$. A saddle-node point exists at $k=$ $k_{c} \approx 0.04618$. (b) Profiles of the unstable single stripe on the lower branch at $k=0.0466$ and $\left(\varphi_{u}, \varphi_{v}\right)$ denotes the profiles of the leading unstable eigenfunction. The $v$-component $\varphi_{v}$ has a dent in the middle that drives the splitting. (c),(d) Response of the unstable single stripe towards negative and positive perturbations of the unstable direction, respectively. By adding a small, positive, constant multiple of the eigenvector to the unstable solution, the stripe begins to split. For a negative perturbation, the system settles on the upper, stable branch. Note that the reference profile of the eigenfuction is depicted in (b) for the sign convention of perturbations. When the model parameter $k$ is moved away from the saddlenode point, the stripe solution initially behaves in stable fashion and then begins to split, guided by the family of unstable manifolds of the unstable branch. 


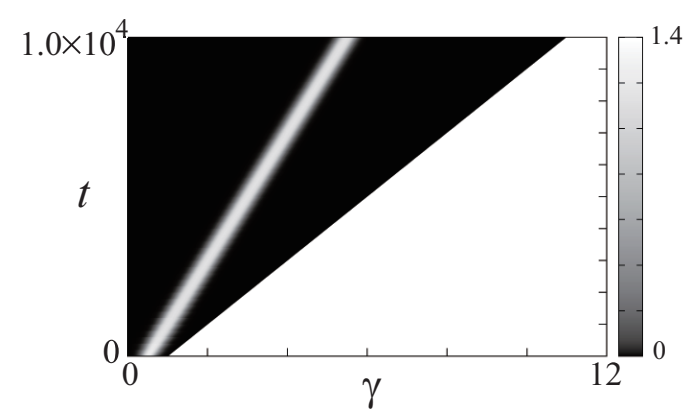

Figure 4: Evolution of (6) on linearly growing domain for $c=1.0 \times 10^{-3}$ and $k=0.048>k_{c}$, where $k_{c}$ is the saddle-node point for an infinite domain. The horizontal axis represents the domain size $\gamma$.

and we take the 1 -stripe solution as initial data; we fix $k=0.041$. Furthermore, in the following numerical simulations, the Neumann boundary conditions are imposed at both ends.

The continuation of stationary solution branches was performed using the Newton method and the resulting bifurcation diagram of $P_{n}$ for $n=1,2, \cdots, 5$ is shown in Fig. 5. In what follows we do not specify $n$, the value of which will be clear from the context and the relevant figures. Because the boundary conditions are of the Neumann type, once $P_{1}$ exists, so do $n$ stripe solutions (for arbitrary $n$ ) for sufficiently large domains. This can be seen by increasing $\gamma$ and using a reflection argument. The branch $P_{n}$ retains $D_{n}$-symmetry when regarded as a solution on the circle [37]. Many asymmetric stationary solutions arise from $n$-stripe solutions via symmetry-breaking bifurcations that are not shown here.

Looking at Fig. 5, each $n$-stripe branch has a saddle-node point $\mathrm{SN}_{n}$, and the $n$-stripe solutions $(n \geq 2)$ lose stability at the $D_{n}$-symmetry breaking bifurcation points $\mathrm{BP}_{n}$ that are found before $\mathrm{SN}_{n}$, whereas the 1-stripe solution loses stability only at $\mathrm{SN}_{1}$. Hereafter, the $i$-th largest eigenvalue corresponding to $P_{n}$ between $\mathrm{BP}_{n}$ and $\mathrm{SN}_{n}$ is referred to as $\lambda_{n}^{i}$, and the eigenfunction corresponding to $\lambda_{n}^{i}$ is referred to as $\varphi_{n}^{i}$. We show numerically in Section 4.4 how $\mathrm{BP}_{n}$ and $\mathrm{SN}_{n}$ affect the dynamics of the stripe-splitting and we study the dependence on growth rate.

\subsection{Spectral behavior along the n-stripe branch}

The behavior of the eigenvalues of the 2-stripe and 3-stripe solutions near a saddle-node point are shown in Figs. 5(d) and (e). There are two real eigenvalues near the origin for the 2-stripe solution. The eigenvalues that cross the imaginary axis at $\mathrm{BP}_{2}$ and $\mathrm{SN}_{2}$ correspond to asymmetric and symmetric instabilities, respectively. Because $\mathrm{BP}_{2}$ is a $D_{2}$-symmetry breaking bifurcation, the eigenfunction corresponding to $\mathrm{BP}_{2}$ is orthogonal to $P_{2}$ [37]. That is, $\left\langle\varphi_{2}^{1}, P_{2}\right\rangle_{L^{2}}=0$, where $\langle\cdot, \cdot\rangle_{L^{2}}$ denotes the $L^{2}$ inner product. The eigenfunction that corresponds to $\mathrm{SN}_{2}$ is not orthogonal to $P_{2}$.

For the 3-stripe solutions, three real eigenvalues exist near the origin. Two of these cross the imaginary axis at $\mathrm{BP}_{3}$ at almost the same time and the other crosses the axis at $\mathrm{SN}_{3}$, i.e., $\mathrm{BP}_{3}$ is a degenerate bifurcation point. In fact, we will show in Section 5 that the bifurcation point $\mathrm{BP}_{n}$ of the principal part for the reduced system is degenerate for $n \geq 3$. Because $\mathrm{BP}_{3}$ corresponds to a $D_{3}$-symmetry breaking bifurcation, the eigenfunctions corresponding to $\mathrm{BP}_{3}\left(\varphi_{3}^{1}\right.$ and $\left.\varphi_{3}^{2}\right)$ are orthogonal to $P_{3}$. The eigenfunction $\varphi_{3}^{3}$ corresponding to $\mathrm{SN}_{3}$ is not orthogonal to $P_{3}$. 
We performed similar numerical treatments for the 4-stripe and 5-stripe solutions, and found eigenvalues and eigenfunctions with the following properties.

(I) The symmetry breaking bifurcation $\mathrm{BP}_{n}$ occurs before $\mathrm{SN}_{n}$. The $n-1$ eigenvalues of $P_{n}$ cross the imaginary axis at almost the same time, and the order of the eigenvalues does not change between $\mathrm{BP}_{n}$ and $\mathrm{SN}_{n}$.

(II) The eigenfunctions corresponding to $\mathrm{BP}_{n}, \varphi_{n}^{i}(i=1,2, \ldots, n-1)$, are orthogonal to $P_{n}$; the eigenfunction corresponding to $\mathrm{SN}_{n}, \varphi_{n}^{n}$, is not orthogonal to $P_{n}$.

(III) The distance between $\mathrm{BP}_{j}$ and $\mathrm{SN}_{j}$ is an increasing function of $j$.

The third property above will become important when we discuss the competition between unstable modes and growth speed. As this distance increases, there is a greater probability that splitting of the alternate type will be observed.

\subsection{Global connection of stable and unstable manifolds}

In order to clarify the behavior of the solution when starting from the neighborhood of an unstable $n$-stripe branch, we now numerically investigate the global behavior of the unstable manifolds that emanate from an $n$-stripe branch. In particular, we focus on the cases where $\gamma$ is between $\mathrm{BP}_{n}$ and $\mathrm{SN}_{n}$, and near $\mathrm{SN}_{n}$.

The global behavior of the unstable manifolds when $\gamma$ is between $\mathrm{BP}_{2}$ and $\mathrm{SN}_{2}$ is calculated by adding a small perturbation of the eigenfunction $\varphi_{2}^{1}$ (Fig.6(b)), which corresponds to the positive eigenvalue. When a negative (positive) perturbation is added, it is observed that the lefthand (right-hand) stripe splits, and the solutions converge to the stable $P_{3}$ configuration (Figs. 6(c) and (d)). In contrast, a numerical treatment of $\varphi_{2}^{2}$ at $\mathrm{SN}_{2}$ confirms that the unstable manifold emanating from $\mathrm{SN}_{2}$ is connected to the stable $P_{4}$ solution (Fig. 6(f)). Although two unstable modes, $\varphi_{2}^{1}$ and $\varphi_{2}^{2}$, exist to the right of $\mathrm{SN}_{2}$ (see Fig. 5(d)), simultaneous splitting is always observed, irrespective of the order of the eigenvalues, because the instability corresponding to $\varphi_{2}^{2}$ grows much faster than that corresponding to $\varphi_{2}^{1}$ as a result of orthogonality (see property (II) in Section 4.2).

As mentioned in Section 4.1, there exist two unstable modes for the 3-stripe solution, $\varphi_{3}^{1}$ and $\varphi_{3}^{2}$ (Fig.7(a)). As a result, four different types of splitting behavior can be observed. By adding small positive and negative perturbations of $\varphi_{3}^{1}$ and $\varphi_{3}^{2}$ to $P_{3}$, we observe the splitting sequences shown in Figs. 7(b)-(e). For the cases Figs. 7(b), (d) and (e), the transitions from $P_{3}$ to $P_{5}$ are observed. On the other hand, for the case Fig. 7(c), the transition from $P_{3}$ to $P_{4}$ is observed. However, because the order of the eigenvalues does not change between $\mathrm{BP}_{3}$ and $\mathrm{SN}_{3}$ (see property (I) above) and both unstable eigenfunctions are orthogonal to $P_{3}$, we generically observe the two types of splitting associated with $\varphi_{3}^{1}$ (Figs. 7(b) and (c)). As for the case of $P_{3}$, simultaneous splitting can be observed to the right of $\mathrm{SN}_{3}$, giving rise to transient dynamics from a 3-stripe to a 6-stripe solution as shown in Fig.7(f).

From similar numerical treatment of $n=4$ and $n=5$ systems, we find that alternate splitting is typically observed when $\gamma$ is between $\mathrm{BP}_{n}$ and $\mathrm{SN}_{n}$, whereas simultaneous splitting is observed for $\gamma$ larger than $\mathrm{SN}_{n}$. That is, we obtain the following prediction for a general $n$-stripe configuration:

(IV) The most unstable mode $\varphi_{n}^{1}$ between $\mathrm{BP}_{n}$ and $\mathrm{SN}_{n}$ is responsible for alternate splitting and the instability $\varphi_{n}^{n}$ is responsible for simultaneous splitting. 


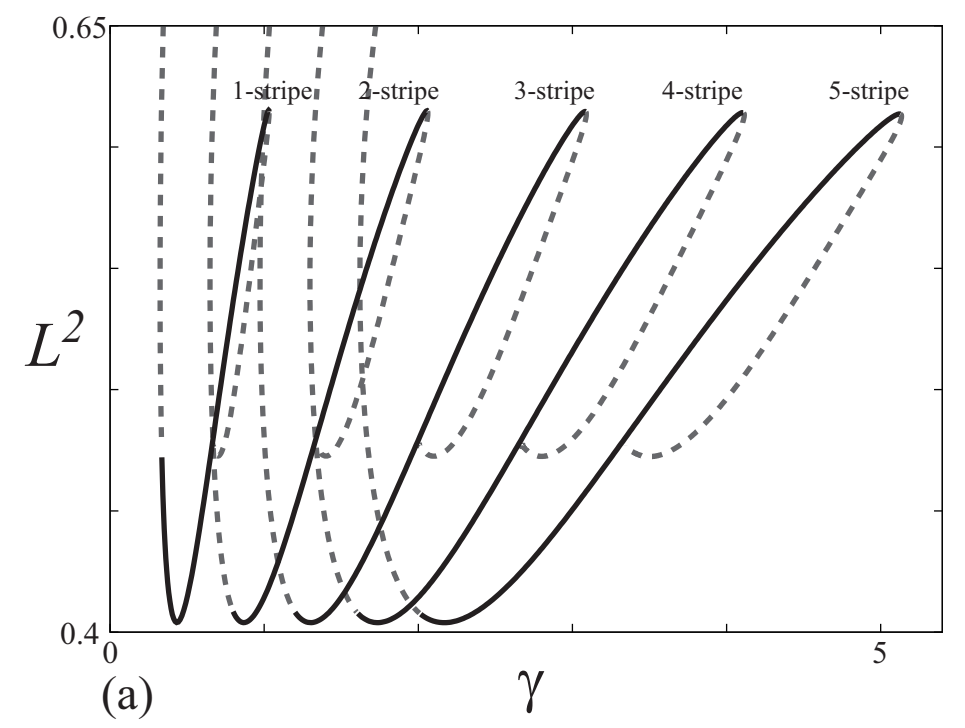

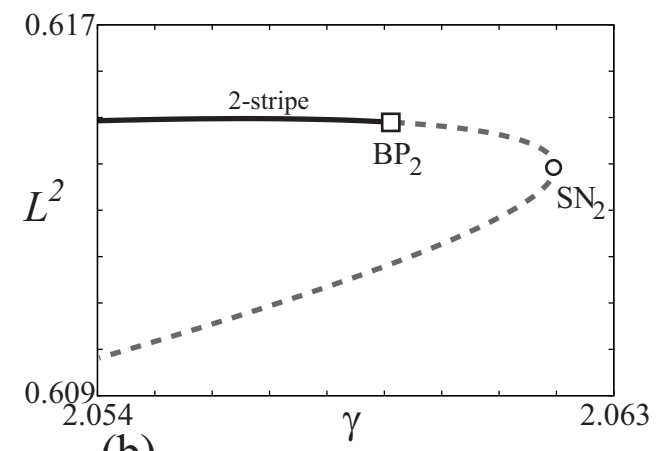

(b)

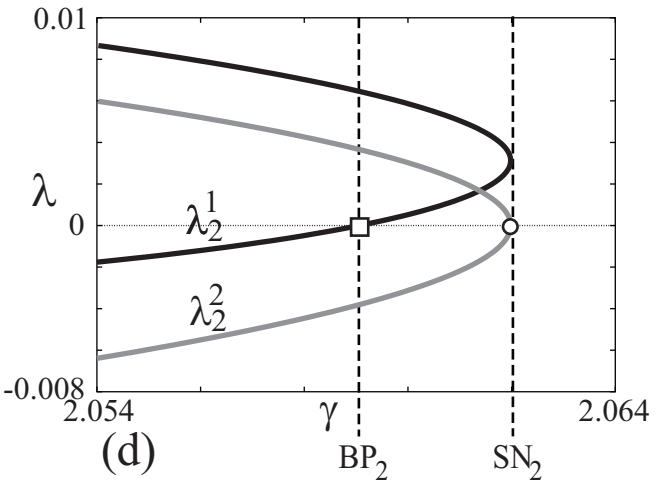

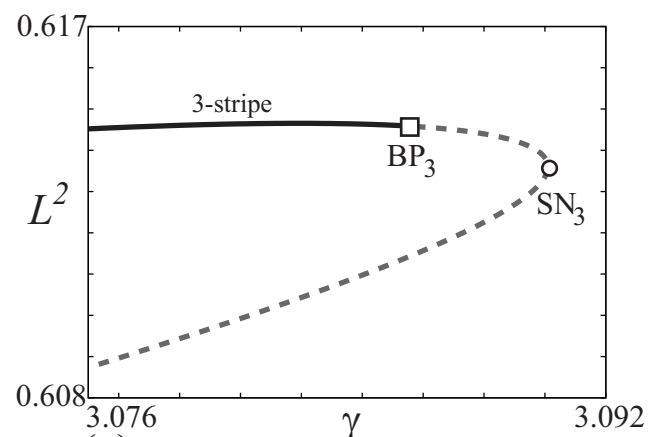

(c)

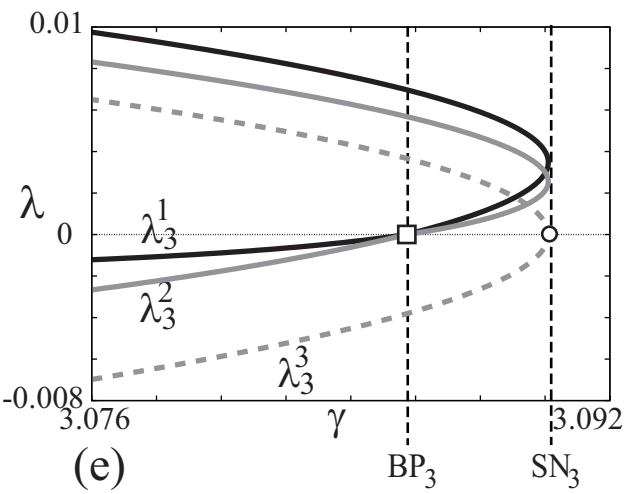

Figure 5: (a) Global bifurcation diagram of $P_{n}$. Solid (dotted) lines indicate stable (unstable) branches. (b) Magnified view of (a) near the saddle-node point of the 2-stripe solution. The symmetry-breaking bifurcation $\left(\mathrm{BP}_{2}\right)$ occurs at $\gamma \approx 2.06$ and the saddle-node (symmetry-preserving) bifurcation $\left(\mathrm{SN}_{2}\right)$ occurs at $\gamma \approx 2.062$. (c) Magnified view of (a) near the saddle-node point of the 3-stripe solution. The symmetry-breaking bifurcation $\left(\mathrm{BP}_{3}\right)$ occurs at $\gamma \approx 3.086$ and the saddle-node (symmetry-preserving) bifurcation $\left(\mathrm{SN}_{3}\right)$ occurs at $\gamma \approx 3.09$. (d), (e) Behavior of the eigenvalues of $P_{2}$ and $P_{3}$, respectively. 

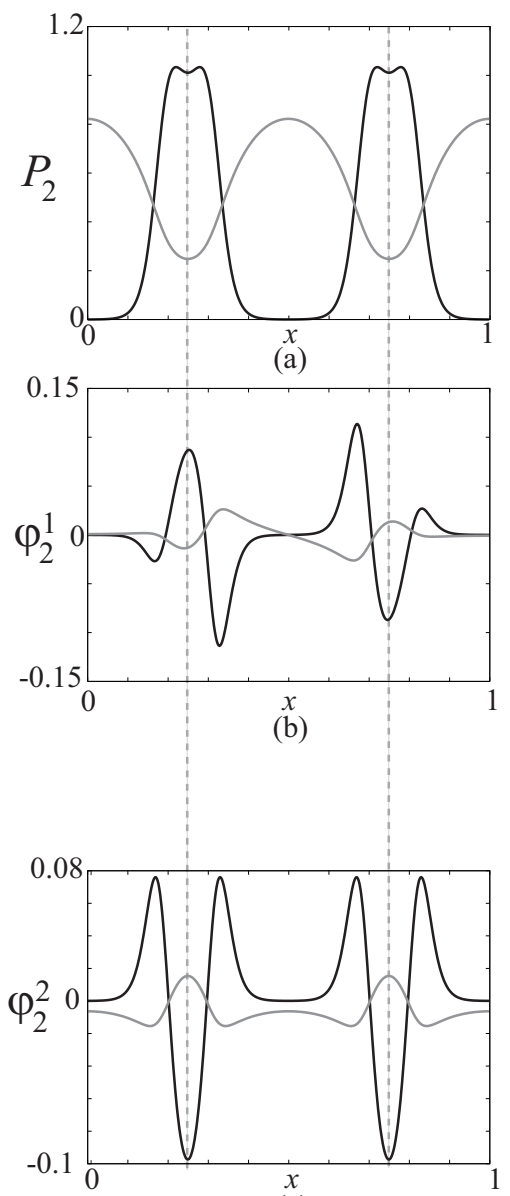

(e)

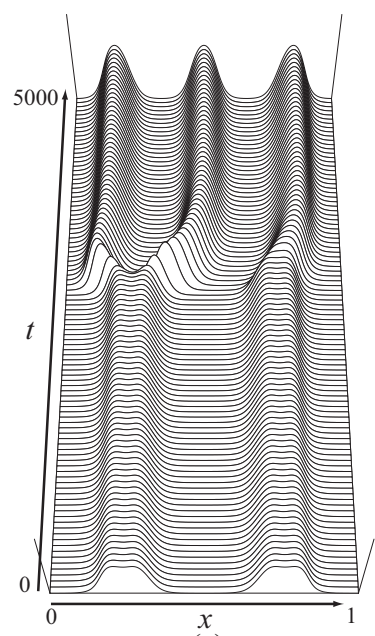

(c)

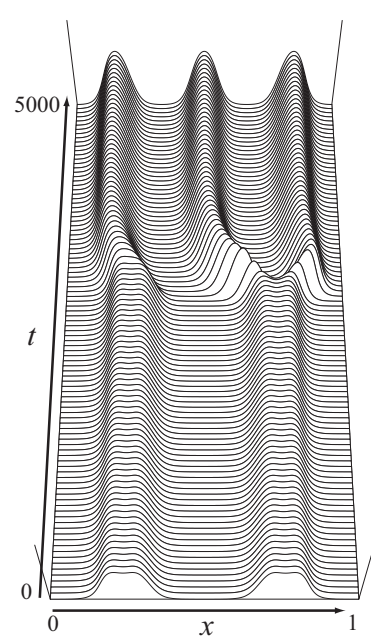

(d)

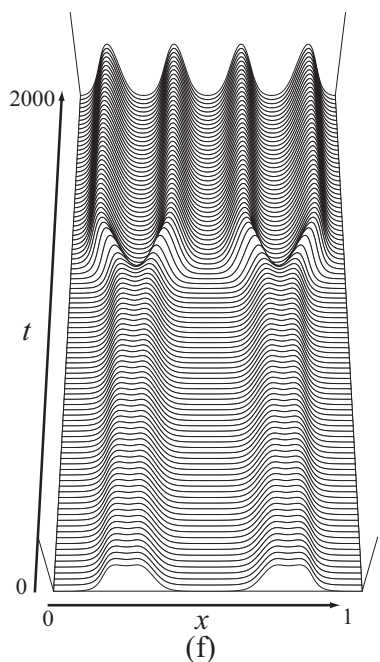

(f)

Figure 6: (a),(b) Profiles of $P_{2}$ and $\varphi_{2}^{1}$ at $\mathrm{BP}_{2}$. (c),(d) Response of unstable $P_{2}$ to negative and positive perturbations of $\varphi_{2}^{1}$, respectively. (e) Profile of $\varphi_{2}^{2}$ at $\mathrm{SN}_{2}$. (f) Response of unstable $P_{2}$ to a positive perturbation of $\varphi_{2}^{2}$. 

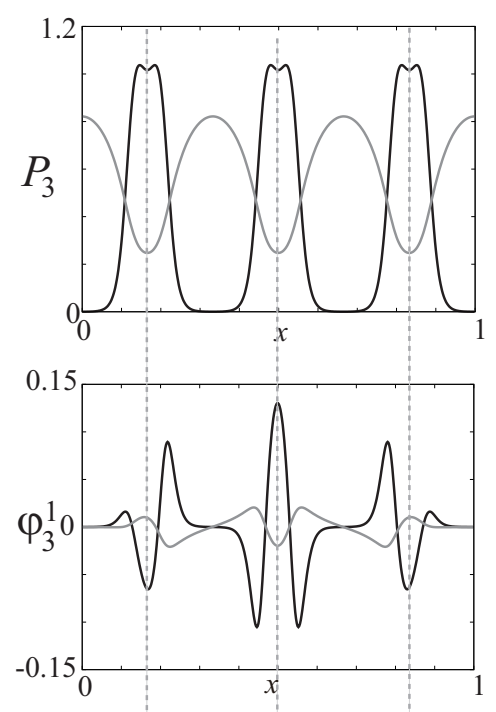

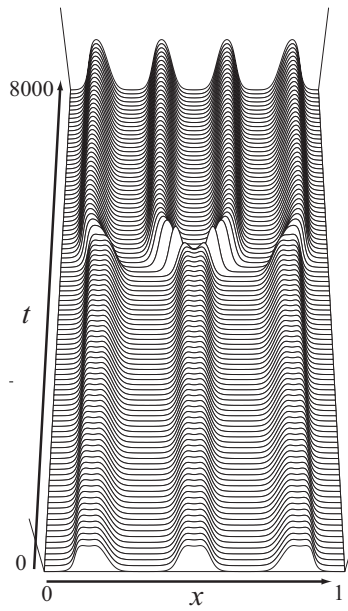

(b)

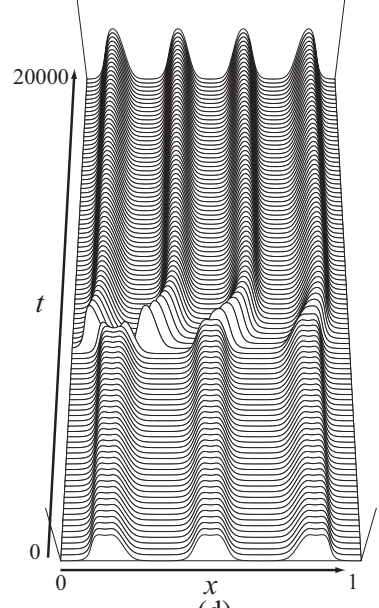

(d)

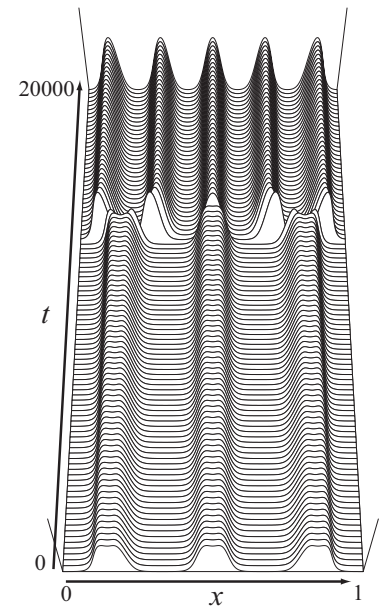

(c)

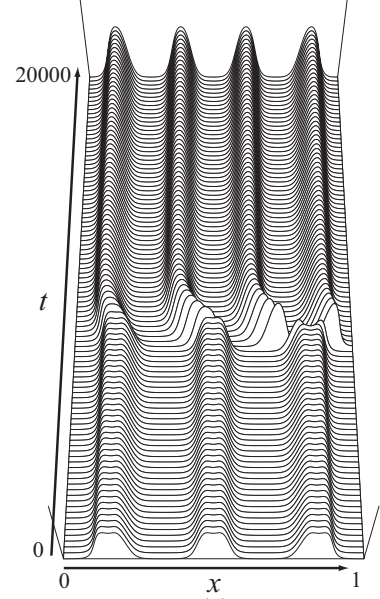

(e)

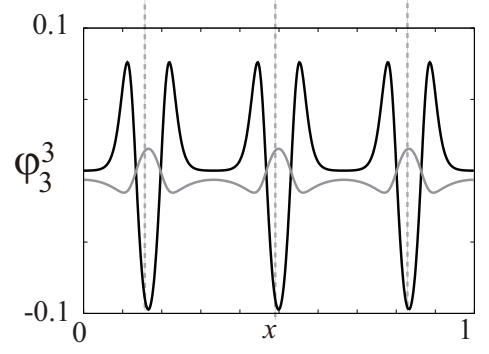

(a)

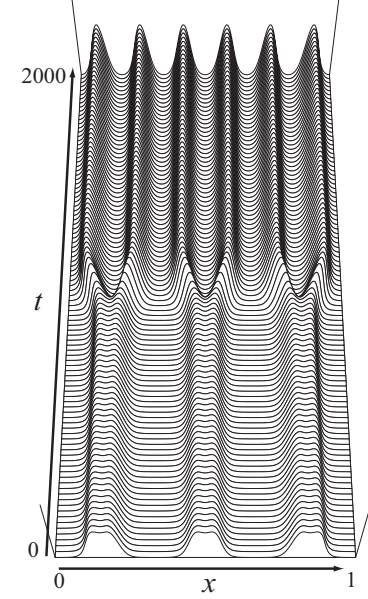

(f)

Figure 7: (a)Profiles of $P_{3}$ and $\varphi_{3}^{j}(j=1,2)$ for $\gamma$ between $\mathrm{BP}_{3}$ and $\mathrm{SN}_{3}(\gamma=3.0866)$, and $\varphi_{3}^{3}$ for $\gamma$ at $\mathrm{SN}_{3}(\gamma=3.0901)$. (b),(c) Response of $P_{3}$ to small negative and positive perturbations of $\varphi_{3}^{1}$, respectively. (d),(e) Response of $P_{3}$ to negative and positive perturbations of $\varphi_{3}^{2}$, respectively. (f) Response of $P_{3}$ to positive perturbations of $\varphi_{3}^{3}$. 


\subsection{Stripe splitting on growing domains}

When the domain growth rate is small enough and the solution is sufficiently close to a stable $n$-stripe branch, a linear stability analysis of the $n$-stripe solutions investigated in subsections 4.14.3 gives us fundamental information about when splitting instabilities begin and how unstable $n$-stripe solutions initially deform. That is, when $c$ is sufficiently small, splitting starts when $\gamma(t)$ reaches $\mathrm{BP}_{n}$ and the deformation of the solution can be characterized by the eigenform corresponding to the most unstable mode of $\mathrm{BP}_{n}$. Figure 8(a) shows a solution trajectory for $c=5.0 \times 10^{-7}$ when $\gamma$ increases sufficiently slowly. As predicted from the linear stability analysis, instabilities of the alternate type occur when $\gamma$ lies between $\mathrm{BP}_{2}$ and $\mathrm{SN}_{2}$, and between $\mathrm{BP}_{3}$ and $\mathrm{SN}_{3}$ (see property (IV))

When the domain growth rate is comparable to or faster than the time-scale of the splitting instability, a linear stability criterion is insufficient to predict the splitting sequence. When the domain growth rate increases to $c=4.0 \times 10^{-5}$, the solution orbit $\gamma(t)$ passes through the unstable region between $\mathrm{BP}_{n}$ and $\mathrm{SN}_{n}$ before asymmetric splitting occurs, and simultaneous splitting eventually takes place at the second and third splitting points near $\mathrm{SN}_{2}$ and $\mathrm{SN}_{4}$, as shown in Fig. 8(d).

It should be noted that, when $\gamma$ is larger than $\mathrm{SN}_{n}$, the instability associated with $\mathrm{SN}_{n}$ dominates the dynamics, irrespective of the order of unstable eigenvalues, because $\mathrm{BP}_{n}$ is an instability of the $D_{n}$-symmetry-breaking type and orthogonal to $P_{n}$ (see property (II)).

It is apparent that the frequency of alternate splitting increases as the time $\gamma(t)$ spent in the unstable regions between $\mathrm{BP}_{n}$ and $\mathrm{SN}_{n}$ increases. Therefore, because of property (III), the transition from simultaneous to alternate splitting in the case of linear growth functions tends to occur more often as $n$ increases, at least at the infinitesimal level. This indicates that a transition from alternate splitting to simultaneous splitting cannot generally be observed for linearly growing domains.

Remark 1. Because $P_{n}$ is orthogonal to $\varphi_{n}^{j}$ for $j=1,2, \ldots, n-1$, we have that $\left\langle\dot{\gamma} / \gamma P_{n}, \varphi_{n}^{j}\right\rangle=$ $\dot{\gamma} / \gamma\left\langle P_{n}, \varphi_{n}^{j}\right\rangle=0$ holds for $j=1,2, \ldots, n-1$. This indicates that the dilution terms in (5) have almost no influence on the symmetry-breaking instabilities when the orbit is sufficiently close to the $P_{n}$ branch. That is, the alternate splitting remains dominant at the infinitesimal level between $B P_{n}$ and $S N_{n}$, regardless of the existence of the advection terms.

In Section 5, we show that the properties (I) to (IV) are valid under appropriate conditions by applying a reduction method to well-separated 1-stripe solutions. The resulting ODE analysis can also be extended to cases with periodic boundary conditions.

\section{Stripe interaction approach to growing domain problem}

We first consider the dynamics of well-separated 1-stripe patterns on an infinite domain near a saddle-node point such that consideration of the domain size parameter $\gamma$ is unnecessary, and we then reduce the PDE dynamics to a problem involving ODEs. Next, we formulate ODEs for wellseparated 1-stripe patterns on a sufficiently large finite domain, using the Neumann or periodic boundary conditions. We then study their spectral behavior as the domain size is increased.

Let us consider the $M$-component system of the form.

$$
\boldsymbol{u}_{t}=\tilde{\mathcal{A}}(\boldsymbol{u} ; \kappa), t>0, x \in \mathbb{R}^{1},
$$




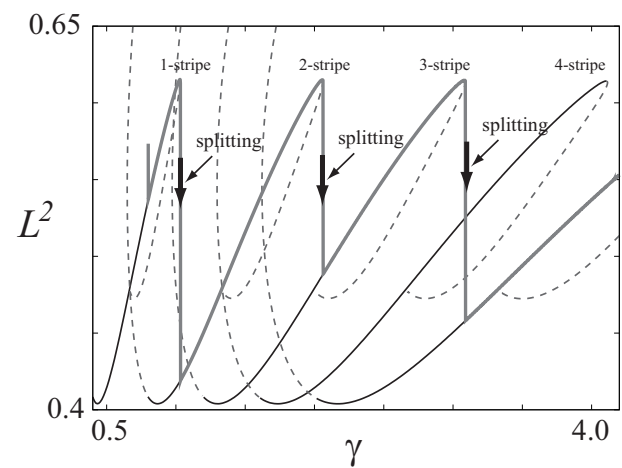

(a)

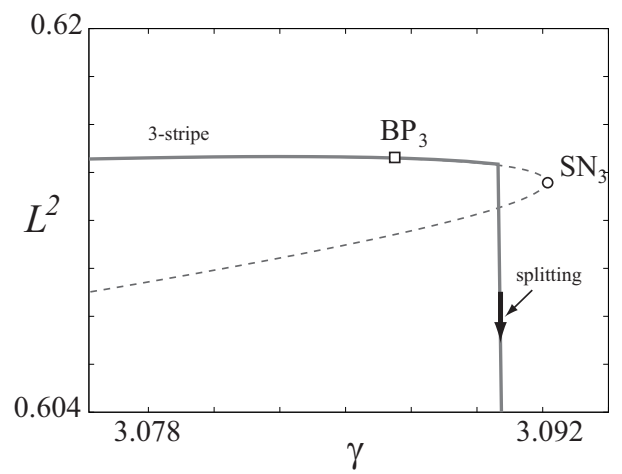

(c)

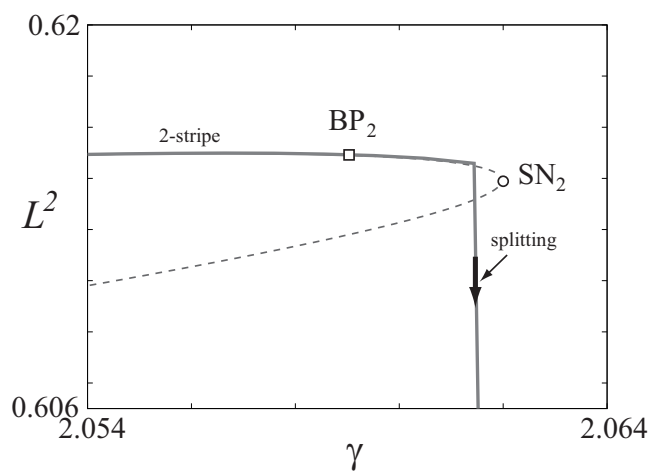

(b)

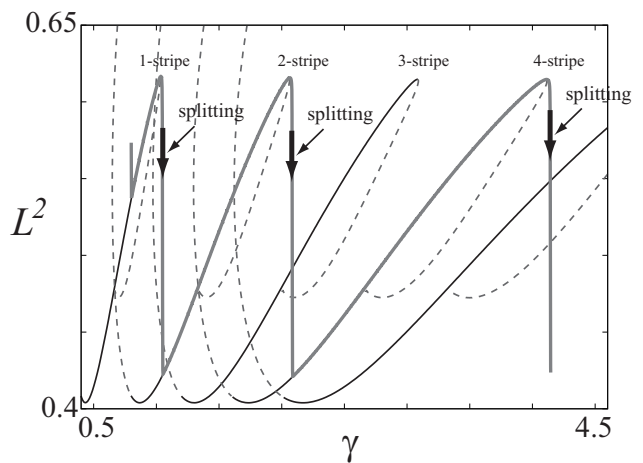

(d)

Figure 8: (a) Trajectory of solution for $c=5.0 \times 10^{-7}$. The trajectory moves along the stable parts of the branches as $\gamma(t)$ increases, then jumps when $\gamma(t)$ lies between $\mathrm{BP}_{n}$ and $\mathrm{SN}_{n}(n=2,3)$. (b),(c) Magnified views of (a) near $\mathrm{SN}_{2}$ and $\mathrm{SN}_{3}$. (d) Trajectory of splitting sequence for $c=4.0 \times 10^{-5}$. Splitting occurs when $\gamma$ is larger than $\operatorname{SN}_{n}(n=1,2,4)$. 
where $\boldsymbol{u}:=\left(u_{0}, u_{1}, \ldots, u_{M-1}\right) \in X:=\left\{L^{2}\left(\mathbb{R}^{1}\right)\right\}^{M}$ with the norm $\|\cdot\|, \widetilde{\mathcal{A}}(\boldsymbol{u} ; \kappa):=D \boldsymbol{u}_{x x}+\widetilde{F}(\boldsymbol{u} ; \kappa)$ and $\kappa \in \mathbb{R}^{1}$ is a bifurcation parameter. $D:=\operatorname{diag}\left(d_{0}, d_{1}, \ldots, d_{M-1}\right)\left(d_{j} \geq 0\right)$ is an $M \times M$ diagonal matrix. We assume (S1)-(S6) with respect to 1-stripe solution on infinite domain, and derive the finite dimensional dynamics as shown in (9) and its principal part (10). All the assumptions can be checked, at least numerically, for the Gray-Scott model (see Remark 2 and [31, 32, 38]).

(S1) $\boldsymbol{u}=\mathbf{0}=(0,0, \ldots, 0) \in X$ is a stable equilibrium of (7) in a neighbourhood of $\kappa=\kappa_{c}$ (see (S2) for the definition of $\left.\kappa_{c}\right)$. That is, $\widetilde{\mathcal{A}}(\mathbf{0} ; \kappa) \equiv \mathbf{0}$ and all the spectrum of the linearized operator $\mathcal{A}^{\prime}(\mathbf{0} ; \kappa)$ lie to the left of the imaginary axis.

For the Gray-Scott model, $(u, v)=(1,0)$ should be shifted to $(0,0)$. The assumption (S1) is used in the proof of Proposition 2 in the Appendix (see [38]).

(S2) The system (7) has a family of stationary solutions parametrized by $\kappa$ with a saddlenode bifurcation point at $\kappa=\kappa_{c}$. The solution $P(x)$ at $\kappa=\kappa_{c}$ has $Z_{2}$-symmetry with respect to inversion $x \rightarrow-x$, namely an even function, and there exists $\alpha>0$ and $\boldsymbol{a}=\left(\tilde{a}_{0}, \tilde{a}_{1}, \ldots, \tilde{a}_{M-1}\right) \in \mathbb{R}^{M}$ such that $P(x) \rightarrow \boldsymbol{a} e^{-\alpha|x|}(x \rightarrow \pm \infty)$.

Let $\widetilde{L}$ be the linearized operator of (7) at the saddle-node point, that is $\widetilde{L}:=\widetilde{\mathcal{A}}^{\prime}\left(P(x), \kappa_{c}\right)$. Let $\xi(x)$ be an eigenfuction at the saddle-node point associated with zero eigenvalue, i.e., $\widetilde{L} \xi=0$, then $\xi$ inherits the $Z_{2}$-symmetry of $P(x)$ and satisfies the following asymptotic behavior $\xi(x) \rightarrow \boldsymbol{b} e^{-\alpha|x|}$ $(x \rightarrow \pm \infty)$, where $\boldsymbol{b}=\left(\tilde{b}_{0}, \tilde{b}_{1}, \ldots, \tilde{b}_{M-1}\right) \in \mathbb{R}^{M}$. Note that there always exists an eigenfunction $P_{x}(x)$ associated with zero eigenvalue coming from the translation invariance, but it does not inherit the $Z_{2}$-symmetry. Next we assume that the above saddle-node bifurcation is primary.

(S3) Let $\Sigma_{c}$ be the spectrum of $\widetilde{L}$. $\Sigma_{c}$ consists of two sets $\Sigma_{0}=\{0\}$ and $\Sigma_{1} \subset\left\{z \in \mathbb{C} ; \operatorname{Re}(z)<-\rho_{0}\right\}$ for a $\rho_{0}>0$, where $\Sigma_{0}$ corresponding to eigenspace consisting of $P_{x}(x)$ and $\xi(x)$.

We consider (7) in the neighborhood of $\kappa=\kappa_{c}$. Suppose $\kappa=\kappa_{c}+\eta$ (where $\eta \in \mathbb{R}$ is a sufficiently small parameter) and rewrite (7) as

$$
\boldsymbol{u}_{t}=\mathcal{A}(\boldsymbol{u})+\eta g(\boldsymbol{u}),
$$

where $\mathcal{A}(\boldsymbol{u}):=\widetilde{\mathcal{A}}\left(\boldsymbol{u} ; \kappa_{c}\right)=D \boldsymbol{u}_{x x}+F(\boldsymbol{u}), F(\boldsymbol{u}):=\widetilde{F}\left(\boldsymbol{u} ; \kappa_{c}\right)$ and $g(\boldsymbol{u}):=\left(\widetilde{\mathcal{A}}\left(\boldsymbol{u} ; \kappa_{c}+\eta\right)-\mathcal{A}(\boldsymbol{u})\right) / \eta$. For $(6), \boldsymbol{u}=(u, v)$ and $g(\boldsymbol{u})=(0,-v)$.

Let $\widetilde{L}^{*}$ be the adjoint operator of $\widetilde{L}$, then there exist two adjoint eigenfunctions $\phi^{*}$ and $\xi^{*}$ associated with the zero eigenvalue of $\widetilde{L}^{*}$, which inherit the same symmetric properties of $P_{x}$ and $\xi$, i.e., $\phi^{*}\left(\xi^{*}\right)$ is an odd (even) function. They also satisfy

$$
\phi^{*}(x) \rightarrow \pm e^{-\alpha|x|} \boldsymbol{a}^{*}, \xi^{*}(x) \rightarrow e^{-\alpha|x|} \boldsymbol{b}^{*}
$$

as $x \rightarrow \pm \infty$, where $\boldsymbol{a}^{*}=\left(\tilde{a}_{0}^{*}, \tilde{a}_{1}^{*}, \ldots, \tilde{a}_{M-1}^{*}\right) \in \mathbb{R}^{M}$ and $\boldsymbol{b}^{*}=\left(\tilde{b}_{0}^{*}, \tilde{b}_{1}^{*}, \ldots, \tilde{b}_{M-1}^{*}\right) \in \mathbb{R}^{M}$.

Let $M_{0}=2 \alpha\left\langle D \boldsymbol{a}, \boldsymbol{a}^{*}\right\rangle, \widetilde{M}_{0}=-2 \alpha\left\langle D \boldsymbol{a}, \boldsymbol{b}^{*}\right\rangle, M_{1}=2 \alpha\left\langle D \boldsymbol{b}, \boldsymbol{a}^{*}\right\rangle, \widetilde{M}_{1}=-2 \alpha\left\langle D \boldsymbol{b}, \boldsymbol{b}^{*}\right\rangle$, and $M_{2}=-M_{0} / \widetilde{M}_{0}\left\langle g(P), \xi^{*}\right\rangle_{L^{2}}$, then

(S4) $M_{0}, \widetilde{M}_{0}, M_{1}$, and $\widetilde{M}_{1}$ are positive.

Note that $\left\langle P_{x}, \xi^{*}\right\rangle_{L^{2}}=\left\langle\xi, \phi^{*}\right\rangle_{L^{2}}=0$ hold because of the symmetry and $\phi^{*}, \xi$, and $\xi^{*}$ are uniquely determined by the following nomalizations:

$$
\left\langle P_{x}, \phi^{*}\right\rangle_{L^{2}}=1,\left\langle\xi, \xi^{*}\right\rangle_{L^{2}}=\frac{\widetilde{M}_{0} M_{1}}{\alpha M_{0}^{2}},\left\langle F^{\prime \prime}(P) \xi \cdot \xi, \xi^{*}\right\rangle_{L^{2}}=\frac{2 \widetilde{M}_{0}}{M_{0}} .
$$


It turns out that the sign of $M_{2}$ determines on which side of $\kappa_{c}$ the branches exist (see Section 5.2 for details). For definiteness we assume here that single 1-stripe branches on infinite domain exist for $\kappa>\kappa_{c}$, namely

(S5) $M_{2}>0$

For the case $M_{2}<0$, the same argument works after change of variables $\kappa \rightarrow-\kappa$.

Remark 2. It has been confirmed numerically that the 1-stripe solution of the Gray-Scott model satisfies the assumptions (S1)-(S6) in Section 5.2. This also holds for the Gierer-Meinhadt model as discussed in Section 6.3. In fact, the bifurcation diagram Fig.3(a) shows numerically that (S3) holds for the Gray-Scott model.

\subsection{Weak interaction of stripes}

Here we introduce a new parameter $\delta$ via $h_{j}$, which now represents the size of the domain instead of $\gamma$. We consider the interaction of $N$ stripes near the saddle-node point. Let

$$
\begin{aligned}
& P(x ; \boldsymbol{h}):=\sum_{j=0}^{N-1} P\left(x-x_{j}\right), \\
& \boldsymbol{\xi}(x ; \boldsymbol{h}, \boldsymbol{r}):=\sum_{j=0}^{N-1} r_{j} \xi\left(x-x_{j}\right), \\
& S(x ; \boldsymbol{h}, \boldsymbol{r}):=\sum_{j=0}^{N-1}\left[P\left(x-x_{j}\right)+r_{j} \xi\left(x-x_{j}\right)\right]=P(x ; \boldsymbol{h})+\boldsymbol{\xi}(x, \boldsymbol{h}, \boldsymbol{r}),
\end{aligned}
$$

where $h_{j}(t):=x_{j}(t)-x_{j-1}(t), x_{j}(t):=x_{0}(t)+\sum_{i=1}^{j} h_{i}(t)(j \geq 1), \boldsymbol{h}:=\left(h_{1}, h_{2}, \ldots, h_{N-1}\right) \in \mathbb{R}^{N-1}$ and $\boldsymbol{r}:=\left(r_{0}, r_{1}, \ldots, r_{N-1}\right) \in \mathbb{R}^{N}$ is the amplitude vector for $\xi$. We define the translation operator $\Xi$ as $\Xi(l) \boldsymbol{u}:=\boldsymbol{u}(x-l)$ and define a set a quantity

$$
\delta(\boldsymbol{h}):=\sup _{x \in \mathbb{R}^{1}}|\mathcal{A}(P(x ; \boldsymbol{h}))| .
$$

We note that $\delta(\boldsymbol{h})=O\left(e^{-\alpha \min \boldsymbol{h}}\right)$ by $(\mathrm{S} 2)$, where $\min \boldsymbol{h}:=\min \left\{h_{1}, h_{2}, \ldots, h_{N-1}\right\}$.

We define $\boldsymbol{q}:=\left(q_{0}, q_{1}, \ldots, q_{N-1}\right) \in \mathbb{R}^{N}$ with $q_{j}:=x_{j}-(\bar{x}+j \bar{h})(j=0, \ldots, N-1)$, which measures the deviation from the equally spaced configuration with distance $\bar{h}$. The values of $\bar{h}$ and $\bar{x}$ will be given later (see Fig. 9). For convenience, we define $\bar{\delta}:=\exp (-\alpha \bar{h})$. We then obtain explicit forms of the equations for $\boldsymbol{q}$ and $\boldsymbol{r}$.

Theorem 1. Assume that assumptions (S1)-(S4) hold. There exist $h^{*}>0$ and $r^{*}>0$ such that as long as $\min \boldsymbol{h}(t)>h^{*}$ and $\max |\boldsymbol{r}|<r^{*}$, then

$$
\left\{\begin{array}{c}
\dot{q}_{j}=-\mathcal{H}_{j}(\boldsymbol{h})-\mathcal{G}_{j}(\boldsymbol{h}, \boldsymbol{r})+O\left(\bar{\delta}^{2}+|\boldsymbol{r}|^{3}+|\eta|^{3 / 2}\right) \\
\dot{r}_{j}=\frac{\alpha M_{0}}{M_{1}}\left(r_{j}^{2}-\eta_{2}+M_{3} \bar{\delta} r_{j}+\frac{M_{0}}{\widetilde{M}_{0}} \widetilde{\mathcal{H}}_{j}(\boldsymbol{q} ; \bar{\delta})-\frac{M_{0}}{\widetilde{M}_{0}} \widetilde{\mathcal{G}}_{j}(\boldsymbol{r} ; \bar{\delta})\right)(j=0,1, \ldots, N-1) \\
+o(\bar{\delta}|\boldsymbol{r}|)+O\left(\bar{\delta}^{2}+|\boldsymbol{r}|^{3}+|\eta|^{3 / 2}\right)
\end{array}\right.
$$


hold for a constant $M_{3} . \mathcal{H}_{j}, \widetilde{\mathcal{H}}_{j}, \mathcal{G}_{j}$ and $\widetilde{\mathcal{G}}_{j}$ are represented by

$$
\begin{aligned}
\mathcal{H}_{j}(\boldsymbol{h}) & :=M_{0}\left(e^{-\alpha h_{j+1}}-e^{-\alpha h_{j}}\right)\left(1+O\left(e^{-\gamma^{\prime} \min } \boldsymbol{h}\right)\right), \\
\widetilde{\mathcal{H}}_{j^{\dagger}}(\boldsymbol{h}) & :=\widetilde{M}_{0}\left(e^{-\alpha h_{j+1}}+e^{-\alpha h_{j}}\right)\left(1+O\left(e^{-\gamma^{\prime} \min } \boldsymbol{h}\right)\right), \\
\mathcal{G}_{j}(\boldsymbol{h}, \boldsymbol{r}) & :=M_{1}\left(r_{j+1} e^{-\alpha h_{j+1}}-r_{j-1} e^{-\alpha h_{j}}\right)\left(1+O\left(e^{-\gamma^{\prime} \min \boldsymbol{h}}\right)\right), \\
\widetilde{\mathcal{G}}_{j^{\dagger}}(\boldsymbol{h}, \boldsymbol{r}) & :=\widetilde{M}_{1}\left(r_{j+1} e^{-\alpha h_{j+1}}+r_{j-1} e^{-\alpha h_{j}}\right)\left(1+O\left(e^{-\gamma^{\prime} \min \boldsymbol{h}}\right)\right) .
\end{aligned}
$$

where $h_{0}=h_{N}=\infty$ and $\gamma^{\prime}>0$.

In the above theorem, the domain is infinite, therefore we impose $h_{0}=h_{N}=\infty$. By taking $h_{0}$ and $h_{N}$ appropriately, we can describe the dynamics of $N$ stripes on the finite interval $[0, L]$ with Neumann or periodic boundary conditions. In fact, we take $h_{0}=2 x_{0}$ and $h_{N}=2\left(L-x_{N-1}\right)$ for the Neumann boundary condition and $h_{0}=h_{N}=x_{0}-\left(x_{N-1}-L\right)$ for periodic boundary condition. Hereafter we set to $\bar{h}=L / N$ and $\bar{x}=\bar{h} / 2$. As long as $\left|q_{j}\right|$ are sufficiently small, $e^{-\alpha h_{j}}=e^{-\alpha \bar{h}}\left(1-\alpha\left(q_{j}-q_{j+1}\right)\right)+O\left(\bar{\delta}|\boldsymbol{q}|^{2}\right)$ hold. Thus, the dynamics of the well-separated stripes are described by the following equations.

$$
\left\{\begin{aligned}
\dot{q}_{j} & =-H_{j}(\boldsymbol{q} ; \bar{\delta})-G_{j}(\boldsymbol{r} ; \bar{\delta})+O\left(\bar{\delta}^{2}+|\boldsymbol{r}|^{3}+|\eta|^{3 / 2}+(|\boldsymbol{q}|+|\boldsymbol{r}|) \delta^{1+\frac{\gamma^{\prime}}{\alpha}}\right), \\
\dot{r}_{j} & =\frac{\alpha M_{0}}{M_{1}}\left(r_{j}^{2}-\eta M_{2}+M_{3} \bar{\delta} r_{j}-\widetilde{H}_{j}(\boldsymbol{q} ; \bar{\delta})-\widetilde{G}_{j}(\boldsymbol{r} ; \bar{\delta})\right) \\
& +o(\bar{\delta}|\boldsymbol{r}|)+O\left(\bar{\delta}^{2}+|\boldsymbol{r}|^{3}+|\eta|^{3 / 2}+(|\boldsymbol{q}|+|\boldsymbol{r}|) \delta^{1+\frac{\gamma^{\prime}}{\alpha}}\right),
\end{aligned} \quad(j=0,1, \ldots, N-1)\right.
$$

hold. $H_{j}, \widetilde{H}_{j}, G_{j}$ and $\widetilde{G}_{j}$ are represented by

$$
\begin{aligned}
& H_{j}(\boldsymbol{q} ; \bar{\delta}):=-\alpha M_{0}\left(q_{j+1}-2 q_{j}+q_{j-1}\right) \bar{\delta} \quad(j=0,1, \ldots, N-1), \\
& \widetilde{H}_{j}(\boldsymbol{q} ; \bar{\delta}):=-2 M_{0}^{\prime} \bar{\delta}+\alpha M_{0}\left(q_{j-1}-q_{j+1}\right) \bar{\delta} \quad(j=0,1, \ldots, N-1), \\
& G_{j}(\boldsymbol{r} ; \bar{\delta}):=M_{1}\left(r_{j+1}-r_{j-1}\right) \bar{\delta} \quad(j=0,1, \ldots, N-1), \\
& \widetilde{G}_{j}(\boldsymbol{r} ; \bar{\delta}):=\frac{M_{0} \widetilde{M}_{1}}{\widetilde{M}_{0}}\left(r_{j-1}+r_{j+1}\right) \bar{\delta} \quad(j=0,1, \ldots, N-1),
\end{aligned}
$$

where $M_{0}^{\prime}=M_{0}+O\left(\delta^{1+\gamma^{\prime} / \alpha}\right)$. We have $q_{-1}=-q_{0}, q_{N}=-q_{N-1}, r_{-1}=r_{0}$, and $r_{N}=r_{N-1}$ for Neumann boundary conditions. For periodic boundary conditions, we have $q_{-1}=q_{N-1}, q_{N}=q_{0}$, $r_{-1}=r_{N-1}$, and $r_{N}=r_{0}$. Since $M_{0}$ is positive from (S4), $M_{0}^{\prime}>0$ for sufficiently small $\delta$. The proof of Theorem 1 is given in the Appendix.

\subsection{Principal part of ODEs}

When the diffusion ratio $D_{u} / D_{v}$ increases for a 2-component system such as (1), the tail of the $v$-component of $P$ decays much faster than that of the $u$-component. In such a case, the interaction between neighboring stripes through the tail of the $v$ component is negligible. In this subsection, we assume that only one component, say $u_{0}$, decays slower than the others. The principal part of (9) then has a simpler form and allows us to compute the stability properties in greater detail. Here, we make the following assumption. 


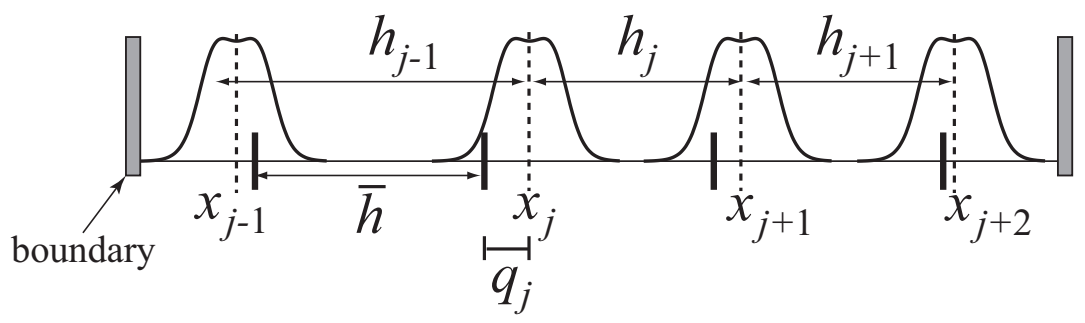

Figure 9: Definition of $h_{j}, \bar{h}, x_{j}$, and $q_{j}$. The variable $q_{j}$ measures the deviation from the equally spaced configuration.

(S6) $\tilde{a}_{0} \neq 0, \tilde{a}_{0}^{*} \neq 0, \tilde{b}_{0} \neq 0, \tilde{b}_{0}^{*} \neq 0$, and $\tilde{a}_{j}=\tilde{a}_{j}^{*}=\tilde{b}_{j}=\tilde{b}_{j}^{*}=0$ for $j=1,2, \ldots, M-1$. Thus, it holds that

$$
\begin{aligned}
& \left\langle D \boldsymbol{a}, \boldsymbol{a}^{*}\right\rangle=d_{0} \tilde{a}_{0} \tilde{a}_{0}^{*}, \\
& \left\langle D \boldsymbol{a}, \boldsymbol{b}^{*}\right\rangle=d_{0} \tilde{a}_{0} \tilde{b}_{0}^{*}, \\
& \left\langle D \boldsymbol{b}, \boldsymbol{a}^{*}\right\rangle=d_{0} \tilde{b}_{0} \tilde{a}_{0}^{*}, \\
& \left\langle D \boldsymbol{b}, \boldsymbol{b}^{*}\right\rangle=d_{0} \tilde{b}_{0} \tilde{b}_{0}^{*} .
\end{aligned}
$$

By (S6), we have

$$
M_{0} / \widetilde{M}_{0}=M_{1} / \widetilde{M}_{1}
$$

We now change variables such that $\tilde{r}_{j}=M_{1}\left(r_{j}+M_{3} \bar{\delta} / 2\right) /\left(\alpha M_{0}\right)$ and $\tilde{t}=\alpha M_{0} t$. Let $N_{1}=$ $\alpha M_{0} / M_{1}^{2}$, and $N_{2}=2 M_{0}^{\prime} /\left(\alpha M_{0}\right), N_{3}=M_{2} / \alpha M_{0}$. From (S4), (S5) and (S6), we note that $N_{1}, N_{2}$ and $N_{3}$ are positive constants. Under assumption (S6), the principal parts of (9), after removing tildes for notational convenience, are given by

$$
\left\{\begin{array}{l}
\dot{q}_{j}=\bar{\delta}\left(q_{j-1}-2 q_{j}+q_{j+1}\right)+\bar{\delta}\left(r_{j-1}-r_{j+1}\right) \\
\dot{r}_{j}=-\bar{\delta}\left(q_{j-1}-q_{j+1}\right)-\bar{\delta}\left(r_{j-1}+r_{j+1}\right)+N_{1} r_{j}^{2}-N_{2} \bar{\delta}-N_{3} \eta
\end{array}\right.
$$

The $n$-stripe solutions of (10) are then given by

$$
q_{j}=\bar{q}(\bar{\delta})=0, \quad r_{j}=\bar{r}_{+}(\bar{\delta}):=\frac{\bar{\delta}+\sqrt{\bar{\delta}^{2}+N_{1}\left(N_{2} \bar{\delta}+N_{3} \eta\right)}}{N_{1}}, \quad(j=0,1, \ldots, N-1),
$$

and

$$
q_{j}=\bar{q}(\bar{\delta})=0, \quad r_{j}=\bar{r}_{-}(\bar{\delta}):=\frac{\bar{\delta}-\sqrt{\bar{\delta}^{2}+N_{1}\left(N_{2} \bar{\delta}+N_{3} \eta\right)}}{N_{1}}, \quad(j=0,1, \ldots, N-1) .
$$

We refer to a branch corresponding to $(\bar{q}(\bar{\delta}), \bar{r}(\bar{\delta}))=\left(0, \bar{r}_{+}(\bar{\delta})\right)$ as an upper branch and $(\bar{q}(\bar{\delta}), \bar{r}(\bar{\delta}))=$ $\left(0, \bar{r}_{-}(\bar{\delta})\right)$ as a lower branch. If $\eta<0$, these two branches coalesce at

$$
\bar{\delta}=\bar{\delta}_{S N}:=\frac{-N_{1} N_{2}+\sqrt{\left(N_{1} N_{2}\right)^{2}-4 N_{1} N_{3} \eta}}{2}
$$


We analyze the linear stability of (10) around $\left(q_{j}, r_{j}\right)=(\bar{q}, \bar{r})$ by taking the domain size $\bar{\delta}$ as a bifurcation parameter with $\eta<0\left(\kappa<\kappa_{c}\right)$.

We make the following definition:

$$
\bar{\delta}_{B P}^{ \pm}:=\frac{N_{1} N_{2} \pm \sqrt{\left(N_{1} N_{2}\right)^{2}+12 N_{1} N_{3} \eta}}{6} .
$$

As will be shown in Theorem 2 and 3, $\bar{\delta}_{S N}$ and $\bar{\delta}_{B P}^{-}$correspond to $\mathrm{SN}_{N}$ and $\mathrm{BP}_{N}$, respectively (Fig. 10). In addition, the bifurcation point $\bar{\delta}_{B P}^{-}$of (10) is degenerated at $\bar{\delta}_{B P}^{-}$. The bifurcation point $\bar{\delta}_{B P}^{+}$is not treated here because $\bar{\delta}_{B P}^{+}$is $O(1)$, i.e., it does not conform to our assumption that $\bar{\delta}$ should be small. By the Taylor expansion, we have

$$
\begin{aligned}
& \bar{\delta}_{S N}=-\frac{N_{3} \eta}{N_{2}}+\frac{N_{3}^{2} \eta^{2}}{N_{1} N_{2}^{3}}+O\left(|\eta|^{3}\right), \\
& \bar{\delta}_{B P}^{-}=-\frac{N_{3} \eta}{N_{2}}+\frac{3 N_{3}^{2} \eta^{2}}{N_{1} N_{2}^{3}}+O\left(|\eta|^{3}\right) .
\end{aligned}
$$

Since $N_{1}>0$ and $N_{2}>0$, we see that $\bar{\delta}_{\mathrm{BP}}^{-}>\bar{\delta}_{\mathrm{SN}}$.

Theorem 2. Assume that (S1) to (S6) hold and $\eta<0$. Let $n_{+}, n_{0}$ and $n_{-}$be the number of positive, zero and negative real eigenvalues of the lower branch of (10) under Neumann boundary conditions. Then

$$
\left(n_{+}, n_{0}, n_{-}\right)= \begin{cases}(0,0,2 N), & \bar{\delta}_{B P}^{-}<\bar{\delta}<\bar{\delta}_{B P}^{+}, \\ (0, N-1, N+1), & \bar{\delta}=\bar{\delta}_{B P}^{-}, \\ (N-1,0, N+1), & \bar{\delta}_{S N}<\bar{\delta}<\bar{\delta}_{B P}^{-}, \\ (N-1,1, N), & \bar{\delta}=\bar{\delta}_{S N},\end{cases}
$$

holds. All eigenvalues are distinct except at $\bar{\delta}=\bar{\delta}_{B P}^{-}$. Let ${ }^{t}\left(q_{0}^{s}, q_{1}^{s}, \ldots, q_{N-1}^{s}, r_{0}^{s}, r_{1}^{s}, \ldots, r_{N-1}^{s}\right)$ be an eigenvector corresponding to the zero eigenvalue for $\bar{\delta}=\bar{\delta}_{S N}$. Then $q_{j}^{s}=0$ and $r_{j}^{s}=1(j=$ $0,1, \ldots, N-1)$. Let ${ }^{t}\left(q_{0}^{l}, q_{1}^{l}, \ldots, q_{N-1}^{l}, r_{0}^{l}, r_{1}^{l}, \ldots, r_{N-1}^{l}\right)(N \geq 2)$ be eigenvectors corresponding to the largest eigenvalue for $\bar{\delta} \in\left[\bar{\delta}_{S N}, \bar{\delta}_{B P}^{-}\right)$. Then $r_{j}^{l}$ and $r_{j+1}^{l}(j=0,1, \ldots, N-2)$ have different signs. All eigenvectors corresponding to positive eigenvalues for $\bar{\delta} \in\left[\bar{\delta}_{S N}, \bar{\delta}_{B P}^{-}\right.$) are orthogonal to (12). Solutions of the upper branch (11) are unstable for $\bar{\delta} \geq \bar{\delta}_{S N}$.

The proof of Theorem 2 is given in the Appendix. From Theorem 2, it is clear that properties (I), (II) and (IV) in Sections 4.2 and 4.3 hold for any $P_{n}$. Moreover, because $r_{j}^{l}$ and $r_{j+1}^{l}$ have different signs, the eigenfunction corresponding to the largest eigenvalue drives the alternate splitting between $\mathrm{BP}_{n}$ and $\mathrm{SN}_{n}$.

Remark 3. From Theorem 2, we see that $n-1$ real eigenvalues of (10) cross the origin simultaneously at $\delta=\delta_{B P}$. That is, the bifurcation point $\delta=\delta_{B P}$ is degenerate (see property (I) in Section 4.2) when (S6) is satisfied. When $M_{0} / \widetilde{M}_{0} \neq M_{1} / \widetilde{M}_{1}$, the ODE system (10) generally has $n-1$ distinct bifurcation points between $B P_{n}$ and $S N_{n}$.

Property (III) in Section 4.2 is directly given by the following Corollary. 
Corollary 1. Let $L_{S N}^{n}$ and $L_{B P}^{n}$ be the domain sizes corresponding to the n-stripe solutions at $\bar{\delta}=\bar{\delta}_{S N}$ and $\bar{\delta}=\bar{\delta}_{B P}^{-}$, respectively. Then,

$$
L_{S N}^{n}-L_{B P}^{n} \propto n
$$

This corollary can be easily proved because $\bar{\delta}_{B P}^{-}$and $\bar{\delta}_{S N}$ are independent of $n$.

Properties (I) to (IV) hold when periodic boundary conditions are imposed. By the following theorem, it is clear that the eigenfunction corresponding to the largest eigenvalue also drives the alternate splitting between $\mathrm{BP}_{n}$ and $\mathrm{SN}_{n}$ for periodic boundary conditions.

Theorem 3. Assume that (S1) to (S6) hold and $\eta<0$. Let $n_{+}, n_{0}$ and $n_{-}$be the number of positive, zero and negative real eigenvalues of the lower branch of (10) under periodic boundary conditions. Then

$$
\left(n_{+}, n_{0}, n_{-}\right)= \begin{cases}(0,1,2 N-1), & \bar{\delta}_{B P}^{-}<\bar{\delta}<\bar{\delta}_{B P}^{+}, \\ (0, N, N), & \bar{\delta}=\bar{\delta}_{B P}^{-}, \\ (N-1,1, N), & \bar{\delta}_{S N}<\bar{\delta}<\bar{\delta}_{B P}^{-}, \\ (N-1,2, N-1), & \bar{\delta}=\bar{\delta}_{S N},\end{cases}
$$

holds. If $N$ is even, there exist one distinct positive eigenvalue and $(N-2) / 2$ pairs of distinct positive eigenvalues for $\bar{\delta} \in\left[\bar{\delta}_{S N}, \bar{\delta}_{B P}^{-}\right)$. If $N$ is odd, there exist $(N-1) / 2$ pairs of distinct positive eigenvalues for $\bar{\delta} \in\left[\bar{\delta}_{S N}, \bar{\delta}_{B P}^{-}\right)$. Let ${ }^{t}\left(q_{0}^{s}, q_{1}^{s}, \ldots, q_{N-1}^{s}, r_{0}^{s}, r_{1}^{s}, \ldots, r_{N-1}^{s}\right)$ be an eigenvector corresponding to the zero eigenvalue for $\bar{\delta}=\bar{\delta}_{S N}$. Then $q_{j}^{s}=0$ and $r_{j}^{s}=1(j=0,1, \ldots, N-1)$. Let ${ }^{t}\left(q_{0}^{l}, q_{1}^{l}, \ldots, q_{N-1}^{l}, r_{0}^{l}, r_{1}^{l}, \ldots, r_{N-1}^{l}\right)(N \geq 2)$ be an eigenvector corresponding to the largest eigenvalue for $\bar{\delta} \in\left(\bar{\delta}_{B P}^{-}, \bar{\delta}_{B P}^{+}\right)$. Then, if $N$ is even, $r_{j}^{l}$ and $r_{j+1}^{l}(j=0,1, \ldots, N-2)$ have different signs. If $N$ is odd, we can take $r_{0}^{l}=0$, and $r_{j}^{l}$ and $r_{j+1}^{l}(j=1,2, \ldots, N-2)$ then have different signs. All eigenvectors corresponding to the positive eigenvalues for $\bar{\delta} \in\left[\bar{\delta}_{S N}, \bar{\delta}_{B P}^{-}\right.$) are orthogonal to (12). The solutions of the upper branch (11) are unstable for $\bar{\delta} \geq \bar{\delta}_{S N}$.

The proof of Theorem 3 is given in the Appendix.

Remark 4. If ${ }^{t}\left(q_{0}^{i}, q_{1}^{i}, \ldots, q_{N-1}^{i}, r_{0}^{i}, r_{1}^{i}, \ldots, r_{N-1}^{i}\right)$ are eigenvectors associated with the linearized problem of (10) under periodic boundary conditions, then an index-rotated eigenvector (e.g. $\left.{ }^{t}\left(q_{N-1}^{i}, q_{0}^{i}, q_{1}^{i} \ldots, q_{N-2}^{i}, r_{N-1}^{i}, r_{0}^{i}, r_{1}^{i}, \ldots, r_{N-2}^{i}\right)\right)$ is also an eigenvector due to rotational invariance.

\section{Discussion}

\subsection{Control of splitting by external perturbations}

It is clear that the dynamics of stripe splitting is rather subtle and that various types of patterns are exhibited, depending on the growth rate. However, it is possible to some extent to control the behavior of stripe splitting based on the results in the previous sections. We will now demonstrate this by using a typical example in which one particular stripe can be split by adding a specific perturbation at an appropriate timing. 
In the following setting, a single stripe located on the right-most side can be made to split in similar fashion to Fig. 7(e), where the secondary instability dominates the dynamics.

$$
\left\{\begin{array}{l}
u_{t}=\frac{D_{u}}{\left(L_{0} \gamma(t)\right)^{2}} u_{x x}-u v^{2}+A(1-u) \\
v_{t}=\frac{D_{v}}{\left(L_{0} \gamma(t)\right)^{2}} v_{x x}+u v^{2}-(A+k) v+g(x, t), \quad x \in[0,1], \quad t \in\left[0, t_{4}\right] . \\
\frac{d \gamma}{d t}=c
\end{array}\right.
$$

Here the perturbation function $g$ consists of three parts and changes its functional form when the domain size reaches $\mathrm{BP}_{i}(i=1,2)$. More precisely,

$$
g(x, t):=\left\{\begin{array}{lll}
0 & \text { for } & 0 \leq t<t_{2} \\
g_{2}(x, t) & \text { for } & t_{2} \leq t<t_{3} \\
g_{3}(x, t) & \text { for } & t_{3} \leq t \leq t_{4}
\end{array}\right.
$$

where (for $n=2,3$ )

$$
g_{n}(x, t):= \begin{cases}1.0 \times 10^{-2}, & \text { for } \frac{n-1}{n}<x \leq 1, \\ 0, & \text { otherwise. }\end{cases}
$$

Here, $t_{n}(n \geq 2)$ denotes the time when $\gamma(t)$ reaches $\mathrm{BP}_{n}$. We note that, because the amplitude of $g_{n}\left(\approx O\left(10^{-2}\right)\right)$ is taken to be much larger than the noise $\left(\approx O\left(10^{-4}\right)\right)$, noise does not play an important role here.

The function $g_{n}$ is spatially localized and has its support on the right-hand interval; it can be numerically confirmed that $\left\langle\varphi_{2}^{1}, g_{2}\right\rangle_{L^{2}}>0$ and $\left\langle\varphi_{3}^{2}, g_{3}\right\rangle_{L^{2}}>\left\langle\varphi_{3}^{1}, g_{3}\right\rangle_{L^{2}}>0$ hold. The former inequality indicates that the right-most stripe is forced to split under the perturbation $g_{2}$ (see Fig. 6(d)). The latter inequality indicates that the secondary instability $\varphi_{3}^{2}$ dominates the dynamics and that the right-most stripe is again forced to split (see Fig. 7(e)). The numerical simulation shown in Fig. 11 agrees with this theoretical prediction.

\subsection{Robust splitting and symmetry}

It is known that under the Neumann conditions, each $n$-stripe solution belongs to a subspace $D_{n}$ of $D_{\infty}$ under reflection and rotation when we regard it as a solution on a circle (see [37] and [39]). The stripe-splitting dynamics starting from a single stripe can be regarded as symmetrycreating rather than symmetry-breaking if we stop the domain growth and then wait for an infinite time for relaxation. However, because the relaxation time for real growth problems is not infinite, such a symmetry-creating process does not occur and each stripe does not belong to $D_{n}$ in a strict sense. There always exists some deviation from $D_{n}$-symmetry that causes symmetry-breaking splitting for slow growth rates.

Crampin et al. [7] found numerically that simultaneous splitting is a robust feature on exponentially growing domains. Furthermore, a similarity law for the solutions was derived in this study by taking appropriate scalings for $x$ and $t$. This was referred to as the matching condition, and was used to explain the robustness of the simultaneous splitting. However, the argument of Crampin et al. is incomplete because it is based on the hypothesis that asymmetric splitting, such 
as splitting from a 2-stripe to a 3-stripe solution, never occurs. In other words, it was supposed that the solution always belongs to a subspace that is symmetric under reflection and rotation. In contrast, we have proved in this paper that asymmetric instabilities $\mathrm{BP}_{n}$ exist under generic assumptions, which implies that asymmetric splitting can be observed even on exponentially growing domains (see Fig. 12(b)). This means that if the asymmetric instabilities can artificially be suppressed until the domain size reaches the saddle-node point, which is responsible for simultaneous splitting, then mode-doubling can robustly be obtained. Otherwise there is always competition between the instabilities and the winner depends on the growth rate and boundary conditions.

\subsection{Universality}

We have employed the Gray-Scott model as being representative of our system, but this does not mean that the numerical and analytical results obtained in this article are specific to this model. In fact, qualitatively similar stripe splitting can be observed in a large class of reactiondiffusion systems.

One such example is the Gierer-Meinhardt model [40], which is described as follows:

$$
\left\{\begin{array}{l}
u_{t}=D_{u} u_{x x}-\mu u+\frac{\rho_{0}\left(u^{2} /\left(1+\rho_{3} u^{2}\right)+\rho_{1}\right)}{v}, \\
v_{t}=D_{v} v_{x x}-k_{1} v+\rho_{0} \frac{u^{2}}{1+\rho_{3} u^{2}}+\rho_{2} .
\end{array}\right.
$$

It is numerically confirmed that a stable, single-stripe solution exists when the domain is sufficiently large and has a saddle-node bifurcation point (Figs. 13(a) and (b)). Furthermore, it is also numerically confirmed that the stationary, single-stripe solution at the saddle-node bifurcation point satisfies assumptions (S1) to (S6). For example, as shown in Fig. 13(b), the decay rate of $v$ is much slower than that of $u$ because $D_{v}$ is taken to be much larger than $D_{u}\left(D_{v} / D_{u}=40\right)$. Therefore, the signs of $M_{0}, \widetilde{M}_{0}, M_{1}$, and $\widetilde{M}_{1}$ are determined by the asymptotic behavior of the profile of $v$. Looking at the profile of the single-stripe solution and the eigenfunctions, we can easily confirm that all of these constants are positive because $\tilde{a}_{0}, \tilde{a}_{0}^{*}$ and $\tilde{b}_{0}$ are positive and $\tilde{b}_{0}^{*}$ is negative.

Numerical simulations of (15) on growing domains exhibit the same qualitative behavior as previously: splitting sequences of type-A, type-M, and type-S are observed and depend on the growth speed (type-A and type-S splitting are shown in Fig. 14).

\subsection{Limitations of theoretical predictions by linearized spectra}

Finally, we discuss the limitations of our theoretical predictions based on the linearized principle. Due to the nature of the linearization, detailed information can be obtained from the spectra in the vicinity of the $P_{n}$-branch. However, when the orbit deviates from the $P_{n}$ branch and enters a nonlinear regime, much more subtle competition exists between the growth speed, the moduli of the associated unstable eigenvalues, and the detailed profiles of the eigenfunctions as well as nonlinearities. This can be illustrated by the splitting from a 4-stripe to a 5-stripe solution shown in Fig. 15(b). As predicted by the linear analysis, the profile of the most unstable mode $\varphi_{4}^{1}$ corresponds to alternate splitting when $\gamma$ is between $\mathrm{BP}_{4}$ and $\mathrm{SN}_{4}$. When a positive perturbation of $\varphi_{4}^{1}$ is added to $P_{4}$, an alternate-type instability grows at the first stage of splitting as in Fig. 15(a). However, our numerics reveals that only the third stripe from the left eventually splits and the leftmost one doesn't; that is, a transition from a 4-stripe to a 5-stripe (not 6-stripe) solution is 


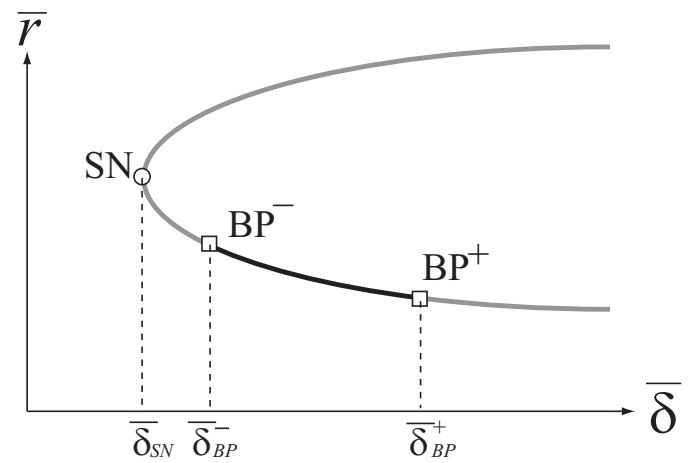

Figure 10: Schematic bifurcation diagram of (10) for $\eta<0 . \mathrm{BP}^{-}$and $\mathrm{SN}$ correspond to $\mathrm{BP}_{N}$ and $\mathrm{SN}_{N}$, respectively.

observed (Fig. 15(a)). Exactly the same breakdown also occurs for the growing domain case, as shown in Fig. 15(b). The profile of the eigenfunction corresponding to the most unstable mode is the key to understanding the mechanism of breakdown of the alternate splitting. The amplitude of the eigenfunction is larger in the central region than in the boundary region as in Fig. 15(a). This implies that the speed of deformation in the central region is larger than that in the boundary region. The difference in speed is small at the infinitesimal level; however, it increases in the nonlinear regime and induces the breakdown of alternate splitting. In fact, as shown in Fig. 15(a), the splitting process involving the third stripe from the left is completed before the left-most stripe starts to split.

\section{Acknowledgment}

We thank the anonymous referees for useful suggestions and comments. This work is partially supported by KAKENHI 21120003, KAKENHI (B) 21340019, and KAKENHI (B) 22740064.

\section{Appendix}

\section{Appendix A. Proofs of Theorems}

Proof of Theorem 1

The proof outlined here is similar to those given in [38] and [32].

Proposition 1 (Proposition 3.1 of [32]). Suppose that assumptions (S1)-(S3) hold. There exist positive constants $C$ and $h^{*}$ such that for $\boldsymbol{h}$ with $\min \boldsymbol{h}>h^{*}$, the operator $\widetilde{L}(\boldsymbol{h})$ has $2 N$ semisimple eigenvalues $\left\{\lambda_{j}(\boldsymbol{h})\right\}_{j=0,1, \ldots, 2 N-1}$ with $\left|\lambda_{j}(\boldsymbol{h})\right| \leq C \delta(\boldsymbol{h})$. Multiple eigenvalues are repeated as many times as their multiplicities indicate. Other spectra of $\widetilde{L}(\boldsymbol{h})$ are located to the left of $z=-\rho_{0}$ for a positive constant $\rho_{0}$.

Let $E(\boldsymbol{h})$ be the eigenspace corresponding to eigenvalues $\left\{\lambda_{j}(\boldsymbol{h})\right\}_{j=0,1, \ldots, 2 N-1}$. The adjoint operator $\widetilde{L}^{*}(\boldsymbol{h})$ also has $2 N$ semi-simple eigenvalues $\left\{\lambda_{j}^{*}(\boldsymbol{h})\right\}_{j=0,1, \ldots, 2 N-1}$ with $\left|\lambda_{j}^{*}(\boldsymbol{h})\right| \leq C \bar{\delta}(\boldsymbol{h})$. Let $E^{*}(\boldsymbol{h})$ be the eigenspace corresponding to eigenvalues $\left\{\lambda_{j}^{*}(\boldsymbol{h})\right\}_{j=0,1, \ldots, 2 N-1}$. 


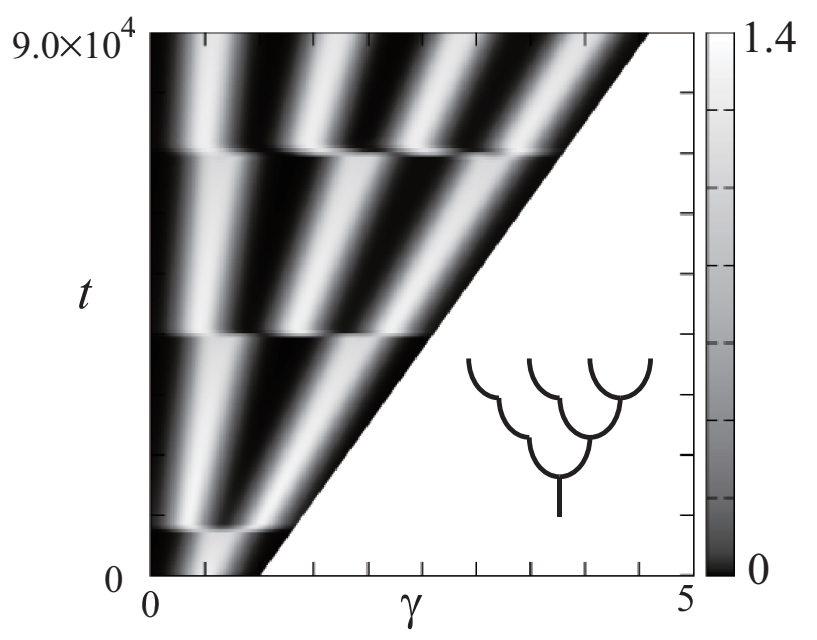

Figure 11: Splitting sequence of (14) for $c=4.0 \times 10^{-5}$.

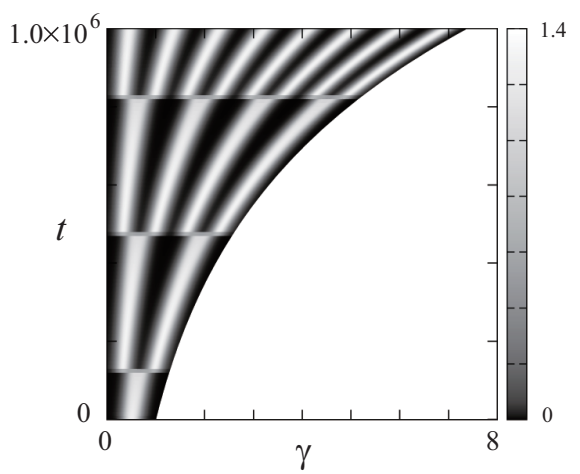

(a)

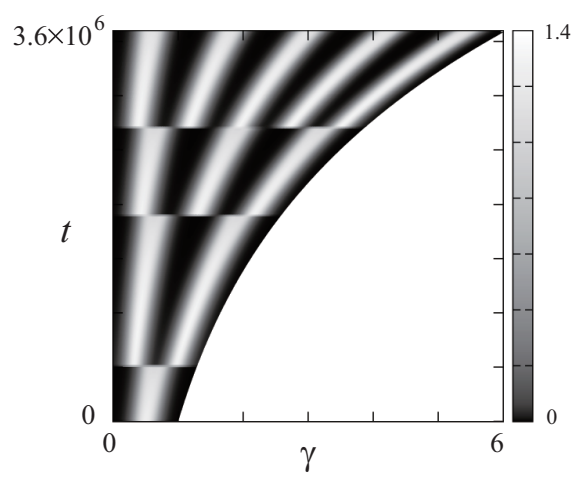

(b)

Figure 12: Splitting sequence of (6) on exponentially growing domains. (a) Simultaneous splitting for $c^{\prime}=2.0 \times 10^{-6}$. (b) Alternate splitting for $c^{\prime}=5.0 \times 10^{-7}$. 


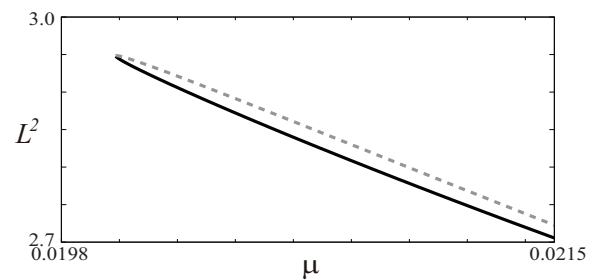

(a)
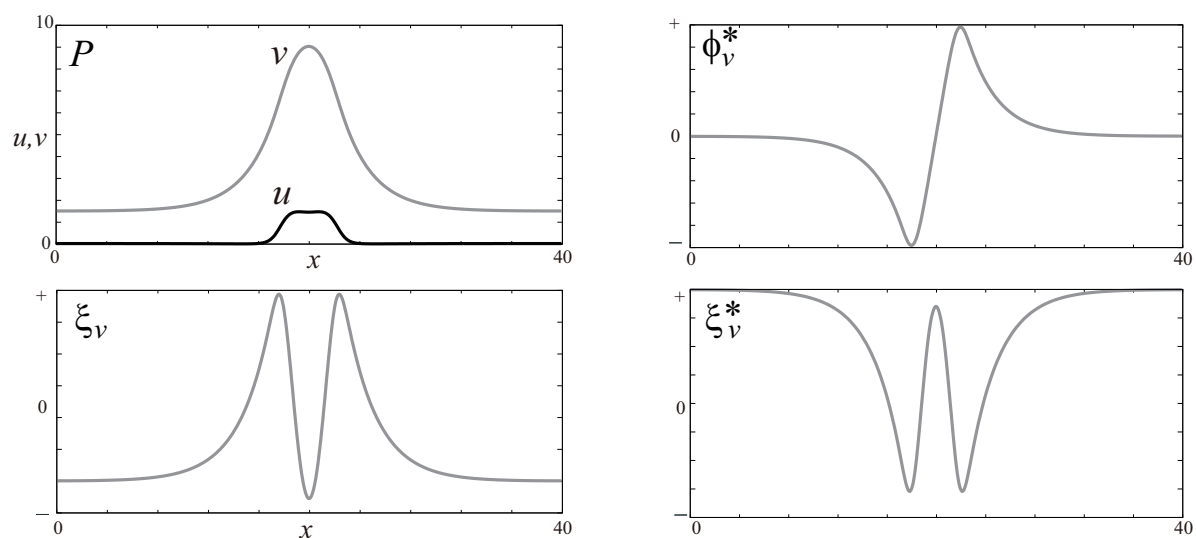

(b)

Figure 13: (a) Bifurcation diagram of single-stripe solution of (15) when $\mu$ is taken as a bifurcation parameter. The saddle-node point is located at $\mu=1.9988 \times 10^{-2}$. (b) Profiles of single-stripe solution $P$ and $v$-components of $\phi^{*}, \xi$ and $\xi^{*}$ at the saddle-node point. The parameters of (15) were set to $D_{u}=0.003, D_{v}=0.12, k_{1}=0.02, \rho_{0}=0.2, \rho_{1}=$ $0.003, \rho_{2}=0.03, \rho_{3}=0.3$, and $L=40$.
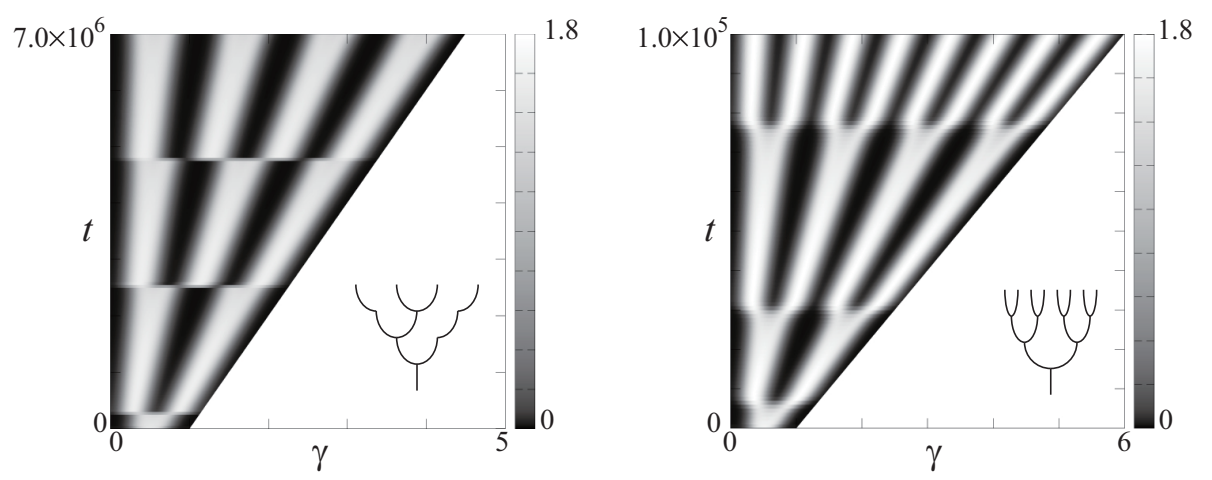

Figure 14: Splitting sequences for (15) when $\mu=0.018$ : alternate splitting for $c=5.0 \times 10^{-7}$ (left) and simultaneous splitting for $c=5.0 \times 10^{-5}$ (right). The profiles of $u$ are displayed. The parameter values $D_{u}, D_{v}, k_{1}, \rho_{0}, \rho_{1}, \rho_{2}$ and $\rho_{3}$ are same as in Fig.13. 

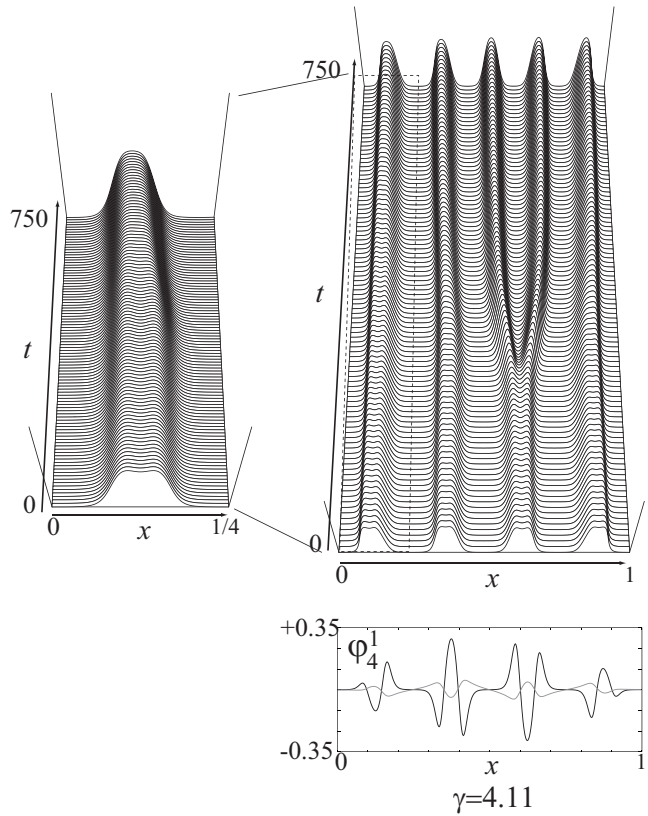

(a)

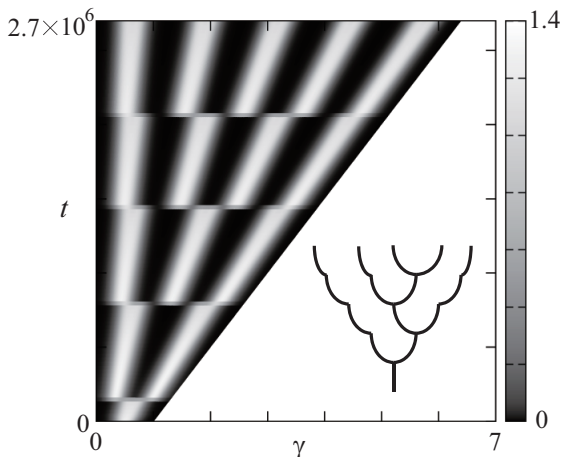

(b)

Figure 15: (a)Perturbation experiments using the Gray-Scott model on a fixed domain for $\gamma=4.11$, which lies between $\mathrm{BP}_{4}$ and $\mathrm{SN}_{4}$. The leftmost hump expands and its middle part becomes slightly dented, although it is a very small deformation even in the magnified inlet. The profile of the eigenfuction $\varphi_{4}^{1}$ corresponding to the most unstable mode (i.e., alternate type) shows the difference of amplitude in the central and boundary regions. (b) A breakdown of alternate splitting on growing domain for $c=2.0 \times 10^{-6}$. 
Proposition 2 (Proposition 3.2 of [32]). Suppose that $(S 1)-(S 3)$ hold. $E(\boldsymbol{h})$ (and $E^{*}(\boldsymbol{h})$ ) are spanned by $2 N$ functions $\left\{\boldsymbol{\phi}_{j}(\boldsymbol{h})(\cdot)\right\}$ and $\left\{\xi_{j}(\boldsymbol{h})(\cdot)\right\}\left(\left\{\boldsymbol{\phi}_{j}^{*}(\boldsymbol{h})(\cdot)\right\}\right.$ and $\left.\left\{\xi_{j}^{*}(\boldsymbol{h})(\cdot)\right\}\right)(j=0,1, \ldots, N-1)$ such that

$$
\begin{aligned}
& \boldsymbol{\phi}_{j}(\boldsymbol{h})(x)=P_{x}\left(x-x_{j}\right)+O(\delta(\boldsymbol{h})), \\
& \xi_{j}(\boldsymbol{h})(x)=\xi\left(x-x_{j}\right)+O(\delta(\boldsymbol{h})), \\
& \boldsymbol{\phi}_{j}^{*}(\boldsymbol{h})(x)=\phi^{*}\left(x-x_{j}\right)+O(\delta(\boldsymbol{h})), \\
& \xi_{j}^{*}(\boldsymbol{h})(x)=\xi^{*}\left(x-x_{j}\right)+O(\delta(\boldsymbol{h})), \\
& \left\langle\boldsymbol{\phi}_{j}(\boldsymbol{h}), \boldsymbol{\phi}_{k}^{*}(\boldsymbol{h})\right\rangle_{L^{2}}=0(j \neq k), \\
& \left\langle\boldsymbol{\phi}_{j}(\boldsymbol{h}), \boldsymbol{\phi}_{j}^{*}(\boldsymbol{h})\right\rangle_{L^{2}}=1
\end{aligned}
$$

hold, where $O(\delta(\boldsymbol{h}))$ means that $\|O(\delta(\boldsymbol{h}))\|_{H^{2}} \leq C \delta(\boldsymbol{h})$.

Let the operator $Q(\boldsymbol{h})$ be the projection from $X$ to $E(\boldsymbol{h})$ and let $R(\boldsymbol{h}):=\operatorname{Id}-Q(\boldsymbol{h})$, where Id is the identity on $X$. Note that $Q$ and $R$ are orthogonal.

Let $E^{\perp}(\boldsymbol{h}):=R(\boldsymbol{h}) X$. Note that $E^{\perp}(\boldsymbol{h})$ is characterized by

$$
E^{\perp}(\boldsymbol{h})=\left\{\boldsymbol{v} \in X ;\left\langle\boldsymbol{v}, \boldsymbol{\phi}_{j}^{*}(\boldsymbol{h})\right\rangle_{L^{2}}=\left\langle\boldsymbol{v}, \xi_{j}^{*}(\boldsymbol{h})\right\rangle_{L^{2}}=0(j=0,1, \ldots, N-1)\right\}
$$

Let $\boldsymbol{h}^{*}:=\left(h^{*}, h^{*}, \ldots, h^{*}\right), \hat{\boldsymbol{h}}:=\left(\hat{h}_{1}, \hat{h}_{2}, \ldots, \hat{h}_{N}\right) \in \mathbb{R}^{N}$ with $\min \hat{\boldsymbol{h}}>h^{*}$. We can then show that there exists a homeomorphism $\Pi(\boldsymbol{h})$ from $E^{\perp}(\hat{\boldsymbol{h}})$ to $E^{\perp}(\boldsymbol{h})$ for $\boldsymbol{h}=\left(h_{1}, h_{2}, \ldots, h_{N}\right) \in \mathbb{R}^{N}$ with $h_{j}>\hat{h}_{j}$ (see Lemma 4.1 of [38]).

Next, we set $\rho_{1}>0$ and define $H\left(\hat{\boldsymbol{h}}, \rho_{1}\right):=\left\{\boldsymbol{h}=\left(h_{1}, h_{2}, \ldots, h_{N}\right) ; \hat{h}_{j}<h_{j}<\hat{h}_{j}+\rho_{1}\right\}$, and $\mathcal{M}=\mathcal{M}\left(\hat{\boldsymbol{h}}, \rho_{1}\right):=\left\{\Xi(l) S(\cdot, \boldsymbol{h}, \boldsymbol{r}) ; l \in \mathbb{R}^{1}, \boldsymbol{h} \in H\left(\hat{\boldsymbol{h}}, \rho_{1}\right),|\boldsymbol{r}|<r^{*}\right\}$. Then there exists a positive constant $C_{1}$ that depends only on $\rho_{1}$ and is independent of $\hat{h}$ with $\hat{h}>h^{*}$ for sufficiently large $h^{*}$, such that for any $\boldsymbol{h} \in H\left(\hat{\boldsymbol{h}}, \rho_{1}\right)$ the map $\Pi(\boldsymbol{h})$ satisfies

$$
\begin{aligned}
& \|\Pi(\boldsymbol{h})\| \leq C_{1},\left\|\Pi^{-1}(\boldsymbol{h})\right\| \leq C_{1},\left\|\frac{\partial}{\partial h_{j}} \Pi(\boldsymbol{h})\right\| \leq C_{1}, \\
& \|\Pi(\boldsymbol{h})\|_{\infty} \leq C_{1},\left\|\Pi^{-1}(\boldsymbol{h})\right\|_{\infty} \leq C_{1},\left\|\frac{\partial}{\partial h_{j}} \Pi(\boldsymbol{h})\right\|_{\infty} \leq C_{1} .
\end{aligned}
$$

Here, $\|\cdot\|_{\infty}$ is the operator norm with respect to the sup-norm $\|\cdot\|_{\infty}$ on $\mathbb{R}^{1}$.

Let $\widetilde{A}=\widetilde{L}(\hat{\boldsymbol{h}})$ and let $X^{\omega}$ be the space with the norm $\|\cdot\|_{\omega}$ defined by the fractional power of $\widetilde{A}^{\omega}$ [41]. We set $\omega$ in the interval $3 / 4<\omega<1$ such that $X^{\omega}$ is embedded into $B U^{1}$, where $B U^{j}\left(\mathbb{R}^{1}\right)$ is the space consisting of uniformly continuous and bounded functions on $\mathbb{R}^{1}$ up to their $j$-th order derivatives.

Proposition 3 (Proposition 3.3 of [32]). There exists a neighborhood $U=U\left(\hat{h}, \rho_{1}\right)$ of $\mathcal{M}\left(\hat{h}, \rho_{1}\right)$ in $X^{\omega}$ such that any $\boldsymbol{u} \in U$ can be uniquely written in the form of

$$
\boldsymbol{u}=\Xi(l)[S(x ; \boldsymbol{h}, \boldsymbol{r})+\Pi(\boldsymbol{h}) \boldsymbol{w}]
$$

for $l \in \mathbb{R}, \boldsymbol{h} \in H\left(\hat{\boldsymbol{h}}, \rho_{1}\right)$ and $\boldsymbol{w} \in E^{\perp}(\hat{\boldsymbol{h}})$.

We transform equation (8) for $\boldsymbol{u}$ to one expressed in terms of $(\boldsymbol{w}, l, \boldsymbol{h}, \boldsymbol{r})$ :

$$
\boldsymbol{u}(t, x)=\Xi(l)[S(x ; \boldsymbol{h}, \boldsymbol{r})+\Pi(\boldsymbol{h}) \boldsymbol{w}]
$$


for $l \in \mathbb{R}^{1}, \boldsymbol{h} \in H\left(\hat{\boldsymbol{h}}, \rho_{1}\right), \max |\boldsymbol{r}|<r^{*}$ and $\boldsymbol{w} \in E^{\perp}(\hat{\boldsymbol{h}})$. Because $\Xi^{\prime}(l)=-\Xi(l) \frac{\partial}{\partial x}$ holds, we obtain

$$
\begin{aligned}
\boldsymbol{u}_{t} & =i \Xi^{\prime}(l)[S(x ; \boldsymbol{h}, \boldsymbol{r})+\Pi(\boldsymbol{h}) \boldsymbol{w}] \\
& +\Xi(l)\left(\frac{\partial}{\partial(\boldsymbol{h}, \boldsymbol{r})}[S(x ; \boldsymbol{h}, \boldsymbol{r})+\Pi(\boldsymbol{h}) \boldsymbol{w}](\dot{\boldsymbol{h}}, \dot{\boldsymbol{r}})+\Pi(\boldsymbol{h}) \boldsymbol{w}_{t}\right) \\
& =\Xi(l)\left(-i \frac{\partial}{\partial x}[S(x ; \boldsymbol{h}, \boldsymbol{r})+\Pi(\boldsymbol{h}) \boldsymbol{w}]+\frac{\partial}{\partial(\boldsymbol{h}, \boldsymbol{r})}[S(x ; \boldsymbol{h}, \boldsymbol{r})+\Pi(\boldsymbol{h}) \boldsymbol{w}](\dot{\boldsymbol{h}}, \dot{\boldsymbol{r}})+\Pi(\boldsymbol{h}) \boldsymbol{w}_{t}\right)
\end{aligned}
$$

and

$$
\begin{aligned}
\mathcal{A}(\boldsymbol{u})+\eta g(\boldsymbol{u}) & =\mathcal{A}(\Xi(l) \hat{\boldsymbol{u}})+\eta g(\Xi(l) \hat{\boldsymbol{u}}) \\
& =\Xi(l)(\mathcal{A}(\hat{\boldsymbol{u}})+\eta g(\hat{\boldsymbol{u}})),
\end{aligned}
$$

where $\hat{\boldsymbol{u}}:=S(x ; \boldsymbol{h}, \boldsymbol{r})+\Pi(\boldsymbol{h}) \boldsymbol{w}$. From (A.1) and (A.2) we can write

$$
-i \frac{\partial}{\partial x}[S(x ; \boldsymbol{h}, \boldsymbol{r})+\Pi(\boldsymbol{h}) \boldsymbol{w}]+\frac{\partial}{\partial(\boldsymbol{h}, \boldsymbol{r})}[S(x ; \boldsymbol{h}, \boldsymbol{r})+\Pi(\boldsymbol{h}) \boldsymbol{w}](\dot{\boldsymbol{h}}, \dot{\boldsymbol{r}})+\Pi(\boldsymbol{h}) \boldsymbol{w}_{t}=\mathcal{A}(\hat{\boldsymbol{u}})+\eta g(\hat{\boldsymbol{u}}) .
$$

By substituting $l=x_{0}$ and defining $P^{\dagger}(\cdot, \boldsymbol{h}):=\Xi\left(-x_{j^{\dagger}}\right) P(\cdot ; \boldsymbol{h}), \boldsymbol{\xi}^{\dagger}(\cdot ; \boldsymbol{h}, \boldsymbol{r}):=\Xi\left(-x_{j^{\dagger}}\right) \boldsymbol{\xi}(\cdot ; \boldsymbol{h}, \boldsymbol{r})$, $S^{\dagger}:=\Xi\left(-x_{j^{\dagger}}\right) S(\cdot, \boldsymbol{h}, \boldsymbol{r})$, and $\boldsymbol{v}^{\dagger}:=\Xi\left(-x_{j^{\dagger}}\right) \Pi(\boldsymbol{h}) \boldsymbol{w}$ into both sides of (A.3), we obtain

$$
\begin{aligned}
& -\frac{\partial}{\partial x}\left[S^{\dagger}(x ; \boldsymbol{h}, \boldsymbol{r})+\boldsymbol{v}^{\dagger}\right] \dot{q}_{0}+\frac{\partial}{\partial(\boldsymbol{h}, \boldsymbol{r})}\left[S^{\dagger}(x ; \boldsymbol{h}, \boldsymbol{r})+\boldsymbol{v}^{\dagger}\right](\dot{\boldsymbol{h}}, \dot{\boldsymbol{r}})+\Pi(\boldsymbol{h}) \boldsymbol{w}_{t} \\
& =\mathcal{A}\left(S^{\dagger}(x ; \boldsymbol{h}, \boldsymbol{r})+\boldsymbol{v}^{\dagger}\right)+\eta g\left(S^{\dagger}(x ; \boldsymbol{h}, \boldsymbol{r})+\boldsymbol{v}^{\dagger}\right) .
\end{aligned}
$$

From Lemma 3.1 of [32], we have

$$
\left|\boldsymbol{v}^{\dagger}(x)\right| \leq O\left(\delta(\boldsymbol{h})+|\boldsymbol{r}|^{2}+|\eta|\right) .
$$

Let $\mathcal{L}(X ; X)$ be the set of bounded bilinear operators from $X$ to $X$. Because $\mathcal{L}(X, \mathcal{L}(X ; X))$ is identified with $\mathcal{L}(X \times X ; X)$, we represent $\left(F^{\prime}(S) \boldsymbol{u}\right)^{\prime} \boldsymbol{v} \in \mathcal{L}(X ; \mathcal{L}(X ; X))(\boldsymbol{u}, \boldsymbol{v} \in X)$ as $F^{\prime \prime}(S) \boldsymbol{u} \cdot \boldsymbol{v} \in$ $\mathcal{L}(X \times X ; X)$, and write $F^{\prime \prime}(S) \boldsymbol{u} \cdot \boldsymbol{u}$ as $F^{\prime \prime}(S) \boldsymbol{u}^{2}$ for simplicity.

We then calculate the inner product of (A.4) with $\boldsymbol{\phi}^{*}(\boldsymbol{h})(x):=\Pi\left(-x_{j^{*}}\right) \boldsymbol{\phi}_{j}^{*}(\boldsymbol{h})(x)$ and $\boldsymbol{\xi}^{*}(\boldsymbol{h})(x)=$ $\Pi\left(-x_{j^{+}}\right) \boldsymbol{\xi}_{j}^{*}(\boldsymbol{h})(x)$. Next, we expand the expression to obtain

$$
\begin{aligned}
& \mathcal{A}\left(S^{\dagger}(x ; \boldsymbol{h}, \boldsymbol{r})+\boldsymbol{v}^{\dagger}\right)+\eta g\left(S^{\dagger}(x ; \boldsymbol{h}, \boldsymbol{r})+\boldsymbol{v}^{\dagger}\right) \\
= & \mathcal{A}\left(P^{\dagger}\right)+\mathcal{A}^{\prime}\left(P^{\dagger}\right) \boldsymbol{\xi}^{\dagger}+\mathcal{A}^{\prime}\left(P^{\dagger}\right) \boldsymbol{v}^{\dagger}+\frac{1}{2} F^{\prime \prime}\left(P^{\dagger}\right)\left(\boldsymbol{\xi}^{\dagger}\right)^{2}+F^{\prime \prime}\left(P^{\dagger}\right) \boldsymbol{\xi}^{\dagger} \cdot \boldsymbol{v}^{\dagger}+\frac{1}{2} F^{\prime \prime}\left(P^{\dagger}\right)\left(\boldsymbol{v}^{\dagger}\right)^{2} \\
& +\eta g\left(P^{\dagger}\right)+\eta g^{\prime}\left(P^{\dagger}\right) \boldsymbol{\xi}^{\dagger}+\frac{1}{2} \eta g^{\prime \prime}\left(P^{\dagger}\right)\left(\boldsymbol{\xi}^{\dagger}\right)^{2}+\eta g^{\prime}\left(P^{\dagger}\right) \boldsymbol{v}^{\dagger}+O\left(\left|\boldsymbol{\xi}^{\dagger}\right|^{3}+\left|\boldsymbol{v}^{\dagger}\right|^{3}+\left|\eta \| \boldsymbol{v}^{\dagger}\right|^{2}\right) .
\end{aligned}
$$


Because $\xi$ is even and $\phi^{*}$ is odd, we have

$$
\begin{aligned}
\left\langle\frac{\partial}{\partial x} S^{\dagger}(x ; \boldsymbol{h}, \boldsymbol{r}), \boldsymbol{\phi}^{*}\right\rangle_{L^{2}} & =\left\langle\frac{\partial}{\partial x} \Xi\left(-x_{j^{\dagger}}\right) S(\cdot, \boldsymbol{h}, \boldsymbol{r}), \boldsymbol{\phi}^{*}\right\rangle_{L^{2}} \\
& =\left\langle\sum_{j=0}^{N-1}\left[P\left(x-x_{j}+x_{j^{\dagger}}\right)+r_{j} \xi\left(x-x_{j}+x_{j^{\dagger}}\right)\right], \boldsymbol{\phi}^{*}\right\rangle_{L^{2}} \\
& =\left\langle P(x)+r_{j^{\dagger}} \xi(x)+\sum_{j=0, j \neq j^{\dagger}}^{N-1}\left[P\left(x-x_{j}+x_{j^{\dagger}}\right)+r_{j} \xi\left(x-x_{j}+x_{j^{\dagger}}\right)\right], \boldsymbol{\phi}^{*}\right\rangle_{L^{2}} \\
& =1+O(\delta(\boldsymbol{h})) .
\end{aligned}
$$

Similarly, because $x_{j^{\dagger}}=x_{0}+\sum_{j=0}^{j^{\dagger}-1} h_{j}$,

$$
\left\langle\frac{\partial}{\partial h_{k}} S^{\dagger}(x ; \boldsymbol{h}, \boldsymbol{r}), \boldsymbol{\phi}^{*}\right\rangle_{L^{2}}= \begin{cases}-1+O(\delta(\boldsymbol{h})) & \left(j^{\dagger} \geq k\right), \\ O(\delta(\boldsymbol{h})) & \left(j^{\dagger}<k\right) .\end{cases}
$$

From Proposition 2, (A.7), (A.8), and $\left\langle\boldsymbol{v}^{\dagger}, \boldsymbol{\phi}^{*}\right\rangle_{L^{2}}=0$, we have

$$
\begin{aligned}
& \left\langle-\frac{\partial}{\partial x}\left[S^{\dagger}(x ; \boldsymbol{h}, \boldsymbol{r})+\boldsymbol{v}^{\dagger}\right] \dot{q}_{0}+\frac{\partial}{\partial(\boldsymbol{h}, \boldsymbol{r})}\left[S^{\dagger}(x ; \boldsymbol{h}, \boldsymbol{r})+\boldsymbol{v}^{\dagger}\right](\dot{\boldsymbol{h}}, \dot{\boldsymbol{r}}), \boldsymbol{\phi}^{*}\right\rangle_{L^{2}} \\
& =-\dot{q}_{0}(1+O(\delta(\boldsymbol{h})))+\sum_{j=1}^{j^{\dagger}} \dot{h}_{j}(1+O(\delta(\boldsymbol{h})))+O(\delta(\boldsymbol{h})(|\dot{\boldsymbol{q}}|+|\dot{\boldsymbol{r}}|)) \\
& =-\dot{q}_{j^{\dagger}}+O(\bar{\delta}(|\dot{\boldsymbol{q}}|+|\dot{\boldsymbol{r}}|)),
\end{aligned}
$$

where we have used $\dot{x}_{0}=\dot{q}_{0}$ and $\sum_{j=1}^{j^{\dagger}} \dot{h}_{j}=-\dot{q}_{0}+\dot{q}_{j^{\dagger}}$. Taking the inner product of the right-hand side of (A.4) (or (A.6)) with $\boldsymbol{\phi}^{*}$, we can write

$$
\begin{aligned}
& \left\langle\mathcal{A}\left(S^{\dagger}(x ; \boldsymbol{h}, \boldsymbol{r})+\boldsymbol{v}^{\dagger}\right)+\eta g\left(S^{\dagger}(x ; \boldsymbol{h}, \boldsymbol{r})+\boldsymbol{v}^{\dagger}\right), \boldsymbol{\phi}^{*}\right\rangle_{L^{2}} \\
& =\mathcal{H}_{j^{\dagger}}(\boldsymbol{h})+\mathcal{G}_{j^{\dagger}}(\boldsymbol{h}, \boldsymbol{r})+O\left(\bar{\delta}^{2}+|\boldsymbol{r}|^{3}+|\eta|^{3 / 2}\right) .
\end{aligned}
$$

where

$$
\mathcal{H}_{j}(\boldsymbol{h})=M_{0}\left(e^{-\alpha h_{j+1}}-e^{-\alpha h_{j}}\right)\left(1+O\left(e^{-\gamma^{\prime} \min \boldsymbol{h}}\right)\right)
$$

for a constant $\gamma^{\prime}>0$. The derivation of $\mathcal{H}_{j}(\boldsymbol{h})$ has been given in the proof of Theorem 2.3 of [38]. Similarly, the function $\mathcal{G}_{j}(\boldsymbol{h}, \boldsymbol{r})$ is given by

$$
\mathcal{G}_{j}(\boldsymbol{h}, \boldsymbol{r})=M_{1}\left(r_{j+1} e^{-\alpha h_{j+1}}-r_{j-1} e^{-\alpha h_{j}}\right)\left(1+O\left(e^{-\gamma^{\prime} \min } \boldsymbol{h}_{)}\right) .\right.
$$

Because

$$
e^{-\alpha h_{j}}=e^{-\alpha \bar{h}}\left(1-\alpha\left(q_{j}-q_{j+1}\right)\right)+O\left(\bar{\delta}|\boldsymbol{q}|^{2}\right),
$$

we use (A.9) and (A.10) to obtain

$$
\dot{q}_{j^{\dagger}}=O\left(\bar{\delta}(|\boldsymbol{q}|+|\boldsymbol{r}|)+\bar{\delta}^{2}+|\eta|^{3 / 2}+\bar{\delta}|\dot{\boldsymbol{r}}|\right) .
$$


Since $h_{j}=\bar{h}+q_{j+1}-q_{j}$, we have

$$
\dot{\boldsymbol{h}}=O\left(\bar{\delta}(|\boldsymbol{q}|+|\boldsymbol{r}|)+\bar{\delta}^{2}+|\eta|^{3 / 2}+\bar{\delta}|\dot{\boldsymbol{r}}|\right) .
$$

Next, we consider the inner product of the right-hand side of (A.4) with $\xi^{*}$. From (A.5), we have

$$
\begin{aligned}
\left\langle F^{\prime \prime}\left(P^{\dagger}\right) \boldsymbol{\xi}^{\dagger} \cdot \boldsymbol{v}^{\dagger}, \boldsymbol{\xi}^{*}\right\rangle_{L^{2}} & =O\left(\bar{\delta}(\boldsymbol{h})+|\boldsymbol{r}|^{2}+|\eta|\right) r_{j^{\dagger}} \\
& \left.=M_{3}^{j^{\dagger}} \bar{\delta} r_{j^{\dagger}}+o(\bar{\delta}|\boldsymbol{r}|)+O\left(|\boldsymbol{r}|^{2}+|\eta|\right)\right),
\end{aligned}
$$

where $M_{3}^{j^{\dagger}} \in \mathbb{R}$. From the symmetry of the $N$-stripe solution (see (S2)), the left-hand side of (A.14) has the same value for all $j^{\dagger}$ if we take $q_{j}=0$ and $r_{j}=r^{\prime} \neq 0(j=0,1, \ldots, N-1)$. Therefore, $M_{3}^{j^{\dagger}}$ is independent of $j^{\dagger}$ and we replace $M_{3}^{j^{\dagger}}$ by $M_{3}^{\prime}$.

From (A.5), we have

$$
\left\langle F^{\prime \prime}\left(P^{\dagger}\right) \boldsymbol{v}^{\dagger^{2}}, \boldsymbol{\xi}^{*}\right\rangle_{L^{2}}=O\left(\bar{\delta}^{2}+|\boldsymbol{r}|^{3}+|\eta|^{3 / 2}\right) .
$$

By taking the inner product of (A.6) with $\xi^{*}$ and dividing the right-hand side by $\widetilde{M}_{0} / M_{0}$, we have from (A.15)

$$
\begin{aligned}
& \frac{M_{0}}{\widetilde{M}_{0}}\left\langle\mathcal{A}\left(S^{\dagger}(x ; \boldsymbol{h}, \boldsymbol{r})+\boldsymbol{v}^{\dagger}\right)+\eta g\left(S^{\dagger}(x ; \boldsymbol{h}, \boldsymbol{r})+\boldsymbol{v}^{\dagger}\right), \boldsymbol{\xi}^{*}\right\rangle_{L^{2}} \\
& =r_{j^{\dagger}}^{2}-\eta M_{2}+M_{3} \bar{\delta} r_{j^{\dagger}}+\frac{M_{0}}{\widetilde{M}_{0}} \widetilde{\mathcal{H}}_{j^{\dagger}}(\boldsymbol{h})-\frac{M_{0}}{\widetilde{M}_{0}} \widetilde{\mathcal{G}}_{j^{\dagger}}(\boldsymbol{h}, \boldsymbol{r})+o(\bar{\delta}|\boldsymbol{r}|)+O\left(\bar{\delta}^{2}+|\boldsymbol{r}|^{3}+|\eta|^{3 / 2}\right),
\end{aligned}
$$

where $M_{3}=M_{0} M_{3}^{\prime} / \widetilde{M}_{0}$ and

$$
\begin{aligned}
\widetilde{\mathcal{H}}_{j^{\dagger}}(\boldsymbol{h}) & =\widetilde{M}_{0}\left(e^{-\alpha h_{j+1}}+e^{-\alpha h_{j}}\right)\left(1+O\left(e^{-\gamma^{\prime} \min } \boldsymbol{h}\right)\right), \\
\widetilde{\mathcal{G}}_{j^{\prime}}(\boldsymbol{h}, \boldsymbol{r}) & =\widetilde{M}_{1}\left(r_{j+1} e^{-\alpha h_{j+1}}+r_{j-1} e^{-\alpha h_{j}}\right)\left(1+O\left(e^{-\gamma^{\prime} \min \boldsymbol{h}}\right)\right) .
\end{aligned}
$$

The derivations of $\widetilde{\mathcal{H}}_{j}(\boldsymbol{h})$ and $\widetilde{\mathcal{G}}_{j}(\boldsymbol{h}, \boldsymbol{r})$ are quite similar to those of (A.11) and (A.12). From the symmetry of the $N$-stripe solution, the left-hand side of (A.17) and (A.18) have the same value for all $j$ if we take $h_{j}=\bar{h}$ and $r_{j}=r^{\prime} \neq 0(j=0,1, \ldots, N-1)$.

Next, we consider the inner product of the left-hand side of (A.4) with $\xi^{*}$. Because $\xi$ and $\xi^{*}$ are even and $P_{x}$ is odd,

$$
\begin{aligned}
\left\langle\frac{\partial}{\partial x} S^{\dagger}(x ; \boldsymbol{h}, \boldsymbol{r}), \boldsymbol{\xi}^{*}\right\rangle_{L^{2}} & =O(\delta(\boldsymbol{h})), \\
\left\langle\frac{\partial}{\partial h_{k}} S^{\dagger}(x ; \boldsymbol{h}, \boldsymbol{r}), \boldsymbol{\xi}^{*}\right\rangle_{L^{2}} & =O(\delta(\boldsymbol{h})) .
\end{aligned}
$$

In similar fashion to (A.9), we use (A.19) and (A.20) to obtain

$$
\begin{aligned}
& \left\langle-\frac{\partial}{\partial x}\left[S^{\dagger}(x ; \boldsymbol{h}, \boldsymbol{r})+\boldsymbol{v}^{\dagger}\right] \dot{q}_{0}+\frac{\partial}{\partial(\boldsymbol{h}, \boldsymbol{r})}\left[S^{\dagger}(x ; \boldsymbol{h}, \boldsymbol{r})+\boldsymbol{v}^{\dagger}\right](\dot{\boldsymbol{h}}, \dot{\boldsymbol{r}}), \boldsymbol{\xi}^{*}\right\rangle_{L^{2}} \\
& =\frac{\widetilde{M}_{0} M_{1}}{\alpha M_{0}^{2}} \dot{j}_{j^{\dagger}}+O(\bar{\delta}(|\dot{\boldsymbol{q}}|+|\dot{\boldsymbol{r}}|)) .
\end{aligned}
$$


From (A.4), (A.13), (A.16) and (A.21), we can write

$$
\begin{aligned}
\dot{r}_{j^{\dagger}} & =\frac{\alpha M_{0}}{M_{1}}\left(r_{j^{\dagger}}^{2}+\eta M_{2}+M_{3} \bar{\delta} r_{j^{\dagger}}+\frac{M_{0}}{\widetilde{M}_{0}} \widetilde{\mathcal{H}}_{j^{\dagger}}(\boldsymbol{q} ; \bar{\delta})-\frac{M_{0}}{\widetilde{M}_{0}} \widetilde{\mathcal{G}}_{j^{\dagger}}(\boldsymbol{r} ; \bar{\delta})\right) \\
& +o(\bar{\delta}|\boldsymbol{r}|)+O\left(\bar{\delta}^{2}+|\boldsymbol{r}|^{3}+|\eta|^{3 / 2}+(|\boldsymbol{q}|+|\boldsymbol{r}|) \delta^{1+\frac{\gamma^{\prime}}{\alpha}}\right) .
\end{aligned}
$$

In order to obtain accurate estimates of $\dot{\boldsymbol{q}}$, we substitute (A.13) and (A.22) into the higher order terms of the right-hand side of (A.9), we obtain

$$
\dot{q}_{j^{\dagger}}=-\mathcal{H}_{j^{\dagger}}(\boldsymbol{h})-\mathcal{G}_{j^{\dagger}}(\boldsymbol{h}, \boldsymbol{r})+O\left(\bar{\delta}^{2}+|\boldsymbol{r}|^{3}+|\eta|^{3 / 2}\right) .
$$

Proof of Theorem 2

We define the variables $y_{j}(t):=q_{j}(t)-r_{j}(t)$ and $z_{j}(t):=q_{j}(t)+r_{j}(t)$. Then, from (10), $\left(y_{0}, z_{0}, y_{1}, z_{1}, \ldots, y_{N-1}, z_{N-1}\right)$ satisfies the following equation:

$$
\left\{\begin{array}{l}
\dot{y}_{j}=\left(2 z_{j-1}-y_{j}-z_{j}\right) \bar{\delta}-N_{1}\left(\frac{y_{j}-z_{j}}{2}\right)^{2}+N_{2} \bar{\delta}+N_{3} \eta, \\
\dot{z}_{j}=\left(2 y_{j+1}-y_{j}-z_{j}\right) \bar{\delta}+N_{1}\left(\frac{y_{j}-z_{j}}{2}\right)^{2}-N_{2} \bar{\delta}-N_{3} \eta,
\end{array} \quad(j=0,1, \ldots, N-1) .\right.
$$

Here, $y_{-1}=-z_{0}, y_{N}=-z_{N-1}, z_{-1}=-y_{0}$, and $z_{N}=-y_{N-1}$ for Neumann boundary conditions, whereas $y_{-1}=y_{N-1}, y_{N}=y_{0}, z_{-1}=z_{N-1}$, and $z_{N}=z_{0}$ for periodic boundary conditions. The $N$-stripe solution, $\left(q_{j}, r_{j}\right)=(\bar{q}, \bar{r})$, is given by

$$
\left(\bar{y}_{0}, \bar{z}_{0}, \bar{y}_{1}, \bar{z}_{1}, \ldots, \bar{y}_{N-1}, \bar{z}_{N-1}\right)=(-\bar{r}, \bar{r},-\bar{r}, \bar{r}, \ldots,-\bar{r}, \bar{r}) .
$$

Let $T_{2 N}$ be the linearized matrix of (A.23) corresponding to $q_{j}=\bar{q}(\bar{\delta})$ and $r_{j}=\bar{r}(\bar{\delta})(j=$ $0,1, \ldots, N-1) . T_{2 N}$ is symmetric tri-diagonal:

$$
T_{2 N}(\bar{\delta})=\left(\begin{array}{ccccccc}
a-b_{0} & b_{1} & & & & & \\
b_{1} & a & b_{0} & & & & \\
& b_{0} & a & b_{1} & & & \\
& & \ddots & \ddots & \ddots & & \\
& & & b_{1} & a & b_{0} & \\
& & & & b_{0} & a & b_{1} \\
& & & & & b_{1} & a-b_{0}
\end{array}\right)
$$

Here, $a=a(\bar{\delta}):=-\bar{\delta}+N_{1} \bar{r}(\bar{\delta}), b_{0}=b_{0}(\bar{\delta}):=2 \bar{\delta}, b_{1}=b_{1}(\bar{\delta}):=-\bar{\delta}-N_{1} \bar{r}(\bar{\delta})$. We note that

$$
a+b_{0}+b_{1}=0
$$

holds for all $\bar{\delta}$. For convenience, we define $a^{-}:=-\bar{\delta}+N_{1} \bar{r}_{-}(\bar{\delta}), b_{1}^{+}:=-\bar{\delta}-N_{1} \bar{r}_{+}(\bar{\delta})$ and $b_{1}^{-}:=-\bar{\delta}-N_{1} \bar{r}_{-}(\bar{\delta})$.

The properties of the eigenvalues and eigenfunctions of tri-diagonal matrices have been studied by Wilkinson [42]. 
Theorem 4 (see [42] p.300 and p.316). Let $C_{n}$ be a symmetric tri-diagonal matrix with nonzero off-diagonal elements of the form

$$
C_{n}=\left(\begin{array}{ccccc}
\alpha_{0} & \beta_{1} & & & \\
\beta_{1} & \alpha_{1} & \beta_{2} & & \\
& \ddots & \ddots & \ddots & \\
& & \beta_{n-2} & \alpha_{n-2} & \beta_{n-1} \\
& & & \beta_{n-1} & \alpha_{n-1}
\end{array}\right), \quad \beta_{i} \neq 0 .
$$

Then $C_{n}$ has $n$ real, distinct, and simple eigenvalues $\lambda_{i}(0 \leq i \leq n-1)$. The eigenvector $\boldsymbol{x}^{i}=$ ${ }^{t}\left(x_{0}^{i}, x_{1}^{i}, \ldots, x_{n-1}^{i}\right)$ corresponding to $\lambda_{i}$ is given by

$$
x_{0}^{i}=1, \quad x_{k}^{i}=(-1)^{k} p_{k}\left(\lambda_{i}\right) / \beta_{1} \beta_{2} \ldots \beta_{k} \quad(1 \leq k \leq n-1),
$$

where $p_{k}(\lambda)$ denotes the leading principal minor of order $k$ of $\left(C_{k}-\lambda I_{k}\right)$ with $p_{0}(\lambda)=1$ :

$$
\begin{aligned}
& p_{1}(\lambda)=\alpha_{0}-\lambda, \\
& p_{j}(\lambda)=\left(\alpha_{j-1}-\lambda\right) p_{j-1}(\lambda)-\beta_{j-1}^{2} p_{j-2}(\lambda) \quad(j=2,3, \ldots, n) .
\end{aligned}
$$

Theorem 5 (see [42] p.300). Let $p_{0}(\mu), p_{1}(\mu), \ldots, p_{n}(\mu)$ be evaluated for some value of $\mu$. Then the number of agreements in sign of successive members of this sequence is the number of eigenvalues of $C_{n}$ that are strictly greater than $\mu$. In particular, if we assume that $\lambda_{l}$ is a maximum eigenvalue of $C_{n}$, then $p_{j}\left(\lambda_{l}\right)$ and $p_{j+1}\left(\lambda_{l}\right)$ have different signs for $j=0,1, \ldots, n-1$.

Regarding the signs of $a^{-}$and $b_{1}^{ \pm}$, we can formulate the following series of lemmas under assumptions (S4), (S5) and (S6) with $\eta<0$.

Lemma 1. Assume that (S4), (S5) and (S6) hold, and $\eta<0$. The signs of $a^{-}$and $b_{1}^{ \pm}$are determined as follows.

$$
\begin{array}{ll}
a^{-}(\bar{\delta})<0, & \text { for } \quad \bar{\delta}>\bar{\delta}_{S N}, \\
a^{-}(\bar{\delta})=0, & \text { for } \quad \bar{\delta}=\bar{\delta}_{S N}, \\
b_{1}^{+}(\bar{\delta})<0, & \text { for } \quad \bar{\delta} \geq \bar{\delta}_{S N}, \\
b_{1}^{-}(\bar{\delta})>0, & \text { for } \quad \bar{\delta}_{B P}^{-}<\bar{\delta}<\bar{\delta}_{B P}^{+}, \\
b_{1}^{-}(\bar{\delta})=0, & \text { for } \quad \bar{\delta}=\bar{\delta}_{B P}^{-}, \\
b_{1}^{-}(\bar{\delta})<0, & \text { for } \quad \bar{\delta}_{S N} \leq \bar{\delta}<\bar{\delta}_{B P}^{-} .
\end{array}
$$

First, we focus on the eigenvalues and eigenfunctions that correspond to the lower branch. The eigenvalues for the 1 -stripe solution $(N=1)$ can be obtained by simple calculation. Thus, we obtain the following lemma.

Lemma 2. The eigenvalues of $T_{2}$ corresponding to the lower branch are $-2 b_{0}(\bar{\delta})$ and $2 a^{-}(\bar{\delta})$.

From Lemmas 1 and 2, we have

$$
\begin{array}{lll}
\left(n_{+}, n_{0}, n_{-}\right)=(0,0,2) & \text { for } & \bar{\delta}>\bar{\delta}_{S N}, \\
\left(n_{+}, n_{0}, n_{-}\right)=(0,1,1) & \text { for } & \bar{\delta}=\bar{\delta}_{S N} .
\end{array}
$$


Next, we consider the $N$-stripe $(N \geq 2)$ eigenvalues.

Lemma 3. If $N \geq 2, T_{2 N}$ corresponding to the lower branch has zero eigenvalues of multiplicity $N-1$ at $\bar{\delta}=\bar{\delta}_{B P}^{-}$and one zero eigenvalue at $\bar{\delta}_{S N}$. There is no zero eigenvalue for $\bar{\delta} \in\left(\bar{\delta}_{S N}, \bar{\delta}_{B P}^{-}\right) \cup$ $\left(\bar{\delta}_{B P}^{-}, \bar{\delta}_{B P}^{+}\right)$.

Proof. From Lemma $1, b_{1}^{-}=0$ for $\bar{\delta}=\bar{\delta}_{B P}^{-}$. Thus, we have

$$
\begin{aligned}
& \left|T_{2 N}\left(\bar{\delta}_{B P}^{-}\right)-\lambda I_{2 N}\right|
\end{aligned}
$$

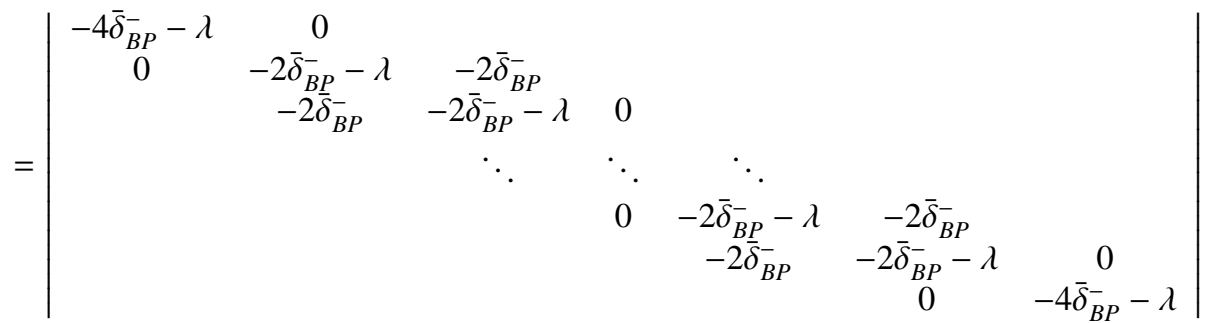

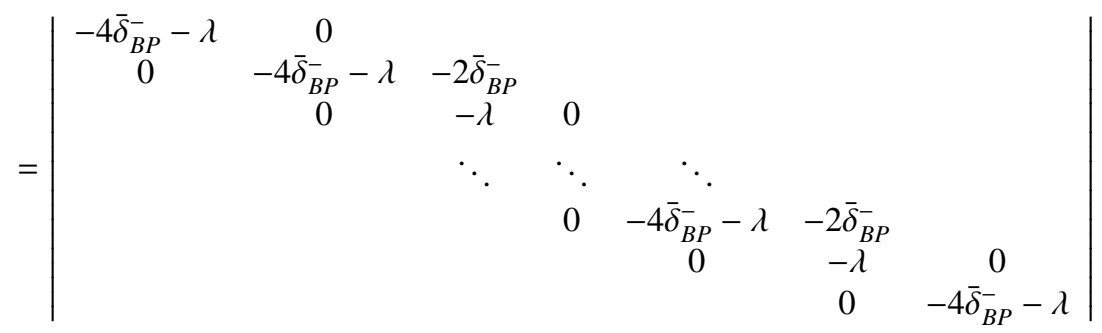

$$
=\lambda^{N-1}\left(-4 \bar{\delta}_{B P}^{-}-\lambda\right)^{N+1}
$$

Here, in order to obtain (A.27), we add the $n+1$-th column of (A.26) to the $n$-th column of (A.26) and then subtract the $n$-th row of (A.26) from the $n+1$-th row of (A.26) for $n=2,4, \cdots, 2(N-1)$. Thus, the eigenvalues of $T_{2 N}\left(\bar{\delta}_{B P}^{-}\right)$are zero with multiplicity $N-1$ and $-4 \bar{\delta}_{B P}^{-}$with multiplicity $N+1$.

Let us consider the number of 0 eigenvalue at $\bar{\delta}=\bar{\delta}_{S N}$. Because $a=0$ at $\bar{\delta}=\bar{\delta}_{S N}$ from 
Lemma 1, we have

$$
\begin{aligned}
& \left|T_{2 N}\left(\bar{\delta}_{S N}\right)-\lambda I_{2 N}\right| \\
& =\left|\begin{array}{ccccccc}
-b_{0}-\lambda & b_{1} & & & & & \\
b_{1} & -\lambda & b_{0} & & & & \\
& b_{0} & -\lambda & b_{1} & & & \\
& & \ddots & \ddots & \ddots & & \\
& & & b_{1} & -\lambda & b_{0} & \\
& & & & b_{0} & -\lambda & b_{1} \\
& & & & & b_{1} & -b_{0}-\lambda
\end{array}\right| \\
& =\left|\begin{array}{ccccccc}
-b_{0}-b_{1}-\lambda & b_{1} & & & & & \\
b_{0}+b_{1}+\lambda & -\lambda & b_{0} & & & & \\
-b_{0}-b_{1}-\lambda & b_{0} & -\lambda & b_{1} & & & \\
\vdots & & \ddots & \ddots & \ddots & & \\
b_{0}+b_{1}+\lambda & & & b_{1} & -\lambda & b_{0} & \\
-b_{0}-b_{1}-\lambda & & & & b_{0} & -\lambda & b_{1} \\
b_{0}+b_{1}+\lambda & & & & & b_{1} & -b_{0}-\lambda
\end{array}\right| \\
& =\left|\begin{array}{ccccccc}
-\lambda & b_{1} & & & & & \\
0 & b_{1}-\lambda & b_{0} & & & & \\
0 & b_{0}-b_{1} & -\lambda & b_{1} & & & \\
\vdots & \vdots & \ddots & \ddots & \ddots & & \\
0 & b_{1} & & b_{1} & -\lambda & b_{0} & \\
0 & -b_{1} & & & b_{0} & -\lambda & b_{1} \\
0 & b_{1} & & & & b_{1} & -b_{0}-\lambda
\end{array}\right| \\
& =-\lambda\left|\begin{array}{cccccc}
b_{1}-\lambda & b_{0} & & & & \\
b_{0}-b_{1} & -\lambda & b_{1} & & & \\
\vdots & \ddots & \ddots & \ddots & & \\
b_{1} & & b_{1} & -\lambda & b_{0} & \\
-b_{1} & & & b_{0} & -\lambda & b_{1} \\
b_{1} & & & & b_{1} & -b_{0}-\lambda
\end{array}\right| .
\end{aligned}
$$

Here in order to obtain (A.29), we subtract the $n$-th column of (A.28) from the 1-st column of (A.28) for $n=2,4, \ldots, 2 N$, and add the $n+1$-th column of (A.28) to the 1-st column of (A.28) for $n=2,4, \ldots, 2(N-1)$. In order to obtain (A.30), we add the 1-st row to the $n$-th row for $n=2,4, \ldots, 2 N$, and subtract the 1-st row from the $n+1$-th row for $n=2,4, \ldots, 2(N-1)$. Therefore $T\left(\bar{\delta}_{S N}\right)$ has 0 eigenvalue at $\bar{\delta}=\bar{\delta}_{S N}$. Since $b_{0} \neq 0$ and $b_{1} \neq 0$ at $\bar{\delta}=\bar{\delta}_{S N}$ from Lemma 1 , it is proved that $T\left(\bar{\delta}_{S N}\right)$ has distinct eigenvalues from Theorem 4; that is, $\bar{\delta}=\bar{\delta}_{S N}$ has only one eigenvalue.

Assume that $T_{2 N}$ has a zero eigenvalue for $\bar{\delta} \in\left(\bar{\delta}_{S N}, \bar{\delta}_{B P}^{-}\right) \cup\left(\bar{\delta}_{B P}^{-}, \bar{\delta}_{B P}^{+}\right)$. Let $\tilde{\boldsymbol{x}}={ }^{t}\left(\tilde{x}_{0}, \tilde{x}_{1}, \ldots, \tilde{x}_{2 N-1}\right) \neq$ $\mathbf{0}$ be an eigenvector corresponding to the zero eigenvalue. If $x_{0} \neq 0$, we can take $x_{0}=1$ without loss of generality. Therefore, because $a^{-}=-b_{0}-b_{1}^{-}$and $T_{2 N} \tilde{\boldsymbol{x}}=\mathbf{0}$, we obtain the following $N$ 
equations.

$$
\begin{aligned}
\tilde{x}_{1}-\tilde{x}_{0}= & \frac{2 b_{0}}{b_{1}^{-}} \tilde{x}_{0}=\frac{2 b_{0}}{b_{1}^{-}}, \\
\tilde{x}_{2}-\tilde{x}_{1}= & \frac{b_{1}^{-}}{b_{0}}\left(\tilde{x}_{1}-\tilde{x}_{0}\right)=2, \\
\tilde{x}_{3}-\tilde{x}_{2}= & \frac{b_{0}}{b_{1}^{-}}\left(\tilde{x}_{2}-\tilde{x}_{1}\right)=\frac{2 b_{0}}{b_{1}^{-}}, \\
& \vdots \\
\tilde{x}_{2 N-2}-\tilde{x}_{2 N-3}= & \frac{b_{1}^{-}}{b_{0}}\left(\tilde{x}_{2 N-3}-\tilde{x}_{2 N-4}\right)=2, \\
\tilde{x}_{2 N-1}-\tilde{x}_{2 N-2}= & \frac{b_{0}}{b_{1}^{-}}\left(\tilde{x}_{2 N-2}-\tilde{x}_{2 N-3}\right)=\frac{2 b_{0}}{b_{1}^{-}}, \\
\tilde{x}_{2 N-1}-\tilde{x}_{2 N-2}= & \frac{-2 b_{0}}{b_{1}^{-}} \tilde{x}_{2 N-1},
\end{aligned}
$$

where we note $b_{0} \neq 0$ and $b_{1}^{-} \neq 0$ from Lemma 1. Equations (A.35) and (A.36) yield $\tilde{x}_{2 N-1}=-1$. We also obtain $\tilde{x}_{2 N-1}=(2 N-1)+(2 N) b_{0} / b_{1}^{-}$by summing equations (A.31) to (A.35). Therefore,

$$
2 N-1+2 N \frac{b_{0}}{b_{1}^{-}}=-1 \text {. }
$$

That is, $b_{0} / b_{1}^{-}=-1$ for $\bar{\delta} \in\left(\bar{\delta}_{S N}, \bar{\delta}_{B P}^{-}\right) \cup\left(\bar{\delta}_{B P}^{-}, \bar{\delta}_{B P}^{+}\right)$, which indicates that $a=b_{0}+b_{1}^{-}+a=0$. However this is a contradiction, since $a \neq 0$ for $\bar{\delta} \in\left(\bar{\delta}_{S N}, \bar{\delta}_{B P}^{-}\right) \cup\left(\bar{\delta}_{B P}^{-}, \bar{\delta}_{B P}^{+}\right)$from Lemma 1.

If $\tilde{x}_{0}=0$, we have $\tilde{x}_{j}=0$ for $j=1,2, \ldots, N-1$ from (A.31) to (A.36), a contradiction. Therefore, $T_{2 N}$ has no zero eigenvalue for $\bar{\delta} \in\left(\bar{\delta}_{S N}, \bar{\delta}_{B P}^{-}\right) \cup\left(\bar{\delta}_{B P}^{-}, \bar{\delta}_{B P}^{+}\right)$.

From Lemmas 1 and 3, we can conclude that $\lambda=2 a(\bar{\delta})$ is the eigenvalue of $T_{2 N}$ corresponding to the saddle-node bifurcation, because $a(\bar{\delta})=a^{-}=0$ at $\bar{\delta}=\bar{\delta}_{S N}$.

By using Theorem 5, Lemma 1 and Lemma 3, we obtain the following lemma.

Lemma 4. Assume that (S4), (S5) and (S6) hold, and $\eta<0$. If $N \geq 2, T_{2 N}$ corresponding to the lower branch has $N-1$ distinct positive eigenvalues for $\bar{\delta} \in\left[\bar{\delta}_{S N}, \bar{\delta}_{B P}^{-}\right)$, and no positive eigenvalues for $\bar{\delta} \in\left[\bar{\delta}_{B P}^{-}, \bar{\delta}_{B P}^{+}\right)$.

Proof. Because $b_{0}>0$ and $b_{1}^{-} \leq 0$ for $\bar{\delta} \in\left[\bar{\delta}_{B P}^{-}, \bar{\delta}_{B P}^{+}\right)$from Lemma 1,

$$
\begin{aligned}
\left\langle\tilde{\boldsymbol{x}}, T_{2 N} \tilde{\boldsymbol{x}}\right\rangle= & -b_{0}\left[2 \tilde{x}_{0}^{2}+\left(\tilde{x}_{1}-\tilde{x}_{2}\right)^{2}+\cdots+\left(\tilde{x}_{2 N-3}-\tilde{x}_{2 N-2}\right)^{2}+2 \tilde{x}_{2 N-1}^{2}\right] \\
& -b_{1}^{-}\left[\left(\tilde{x}_{0}-\tilde{x}_{1}\right)^{2}+\cdots+\left(\tilde{x}_{2 N-2}-\tilde{x}_{2 N-1}\right)^{2}\right]<0,
\end{aligned}
$$

which implies that the maximum eigenvalue of the symmetric matrix $T_{2 N}(N \geq 2)$ is not positive for $\bar{\delta} \in\left[\bar{\delta}_{B P}^{-}, \bar{\delta}_{B P}^{+}\right)$.

From Lemma $3, \lambda=2 a(\bar{\delta})$ is an eigenvalue of $T_{2 N}$ that is equal to 0 at the saddle-node point $\bar{\delta}=\bar{\delta}_{S N}$. The sequences of $\tilde{p}_{k}$, the leading principal minor of order $k$ of $\left(T_{k}-\lambda I_{k}\right)$ with $\tilde{p}_{0}(\lambda)=1$, 
given by (A.24) and (A.25) for $\bar{\delta}=\bar{\delta}_{S N}$, yield

$$
\begin{aligned}
\tilde{p}_{2 N}(0) & =0, \\
\tilde{p}_{2 j+1}(0) & =-\left(b_{0}\right)^{2} \tilde{p}_{2 j-1}(0) \quad(j=1,2, \ldots, N-1), \\
\tilde{p}_{2 j}(0) & =-\left(b_{1}^{-}\right)^{2} \tilde{p}_{2 j-2}(0) \quad(j=1,2, \ldots, N-1), \\
\tilde{p}_{1}(0) & =-b_{0} .
\end{aligned}
$$

Therefore, the signs of $\tilde{p}_{k}$ are determined:

$$
\begin{aligned}
& \tilde{p}_{0}(0)=1>0, \\
& \tilde{p}_{1}(0)=-b_{0}<0, \\
& \tilde{p}_{2 j}(0)>0, \quad(j \text { is even and } j \leq N-1) \\
& \tilde{p}_{2 j}(0)<0, \quad(j \text { is odd and } j \leq N-1) \\
& \tilde{p}_{2 j-1}(0)>0, \quad(j \text { is even and } j \leq N) \\
& \tilde{p}_{2 j-1}(0)<0, \quad(j \text { is odd and } j \leq N) \\
& \tilde{p}_{2 N}(0)=0 .
\end{aligned}
$$

Thus, from Theorem 5 there exist $N-1$ positive eigenvalues, $N$ negative eigenvalues and one zero eigenvalue at $\bar{\delta}=\bar{\delta}_{S N}$.

From Lemma $3, T_{2 N}(\bar{\delta})$ has no zero eigenvalue for $\bar{\delta}_{S N}<\bar{\delta}<\bar{\delta}_{B P}^{-}$and $T_{2 N}(\bar{\delta})$ has a zero eigenvalue with multiplicity $N-1$ at $\bar{\delta}_{B P}^{-}$, which implies that there exist $N-1$ positive eigenvalues for $\bar{\delta} \in\left[\bar{\delta}_{S N}, \bar{\delta}_{B P}^{-}\right)$.

From Lemmas 2 to 4 , we obtain (13). The following Lemma 5, regarding the eigenfunctions corresponding to the saddle-node bifurcation, can be proved easily.

Lemma 5. The eigenfunction of $T_{2 N}$ corresponding to $\lambda=2 a^{-}$is given by $q_{j}^{s}=0, r_{j}^{s}=1$ $(j=0,1, \ldots, N-1)$.

The signs of $r_{j}^{l}$ are given by the following lemma.

Lemma 6. Assume that (S4), (S5) and (S6) hold, and $\eta<0$. For $\bar{\delta} \in\left[\bar{\delta}_{S N}, \bar{\delta}_{B P}^{-}\right), r_{j}^{l}$ and $r_{j+1}^{l}$ have different signs for $j=0,1, \ldots, N-2$.

Proof. From Theorem 4, the eigenvectors corresponding to $\lambda$ for $\bar{\delta} \neq \bar{\delta}_{B P}^{ \pm}$are given by

$$
y_{j}=\frac{\tilde{p}_{2 j}(\lambda)}{\left(b_{0}\right)^{j}\left(b_{1}^{-}\right)^{j}}, z_{j}=\frac{-\tilde{p}_{2 j+1}(\lambda)}{\left(b_{0}\right)^{j}\left(b_{1}^{-}\right)^{j+1}} \quad(j=0,1, \ldots, N-1) .
$$

From Theorem 5, the signs of $\tilde{p}_{k}\left(\lambda_{l}\right)$ and $\tilde{p}_{k+1}\left(\lambda_{l}\right)(k=0,1, \ldots, 2 N-2)$ are different for $\bar{\delta} \epsilon$ $\left(\bar{\delta}_{S N}, \bar{\delta}_{B P}^{-}\right)$. Because $b_{1}^{-}$is negative for $\bar{\delta} \in\left[\bar{\delta}_{S N}, \bar{\delta}_{B P}^{-}\right)$from Lemma 1 , we can conclude from (A.37) that $r_{j}^{l}=\left(y_{j}^{l}-z_{j}^{l}\right) / 2$ and $r_{j+1}^{l}=\left(y_{j+1}^{l}-z_{j+1}^{l}\right) / 2$ have different signs.

The orthogonality between the eigenvectors corresponding to $\mathrm{BP}_{n}$ and $N$ stripes is guaranteed by the following Lemma.

Lemma 7. Let $\tilde{\boldsymbol{x}}=\left(\tilde{x}_{0}, \tilde{x}_{1}, \ldots, \tilde{x}_{2 N-1}\right)$ be an eigenvector of $T_{2 N}$ corresponding to the eigenvalue $\lambda \neq 2 a(\bar{\delta})$. Then $\tilde{\boldsymbol{x}}$ and $\left(\bar{y}_{0}, \bar{z}_{0}, \bar{y}_{1}, \bar{z}_{1}, \ldots, \bar{y}_{N-1}, \bar{z}_{N-1}\right)$ are orthogonal. 
Proof. Because $T_{2 N} \tilde{\boldsymbol{x}}=\lambda \tilde{\boldsymbol{x}}$, we have

$$
\sum_{i=0}^{2 N-1}(-1)^{i}\left(a-b_{0}-b_{1}\right) \tilde{x}_{i}=\lambda \sum_{i=0}^{2 N-1}(-1)^{i} \tilde{x}_{i}
$$

Because $a+b_{0}+b_{1}=0$, it follows that

$$
(2 a-\lambda) \sum_{i=0}^{2 N-1}(-1)^{i} \tilde{x}_{i}=0 .
$$

Therefore, $\tilde{\boldsymbol{x}}$ and $\left(\bar{y}_{0}, \bar{z}_{0}, \bar{y}_{1}, \bar{z}_{1}, \ldots, \bar{y}_{N-1}, \bar{z}_{N-1}\right)=-(1,-1,1,-1, \ldots, 1,-1)$ are orthogonal when $\lambda \neq 2 a$.

From Lemma 3, the eigenvector corresponding to $\lambda=2 a(\bar{\delta})$ is responsible for the saddle-node bifurcation, hence Lemma 7 yields the result that the unstable eigenvectors for $\bar{\delta} \in\left(\bar{\delta}_{S N}, \bar{\delta}_{B P}^{-}\right)$are orthogonal to (12).

Finally, we have the following Lemma 8 concerning the stability of the upper branch.

Lemma 8. Assume that (S4), (S5) and (S6) hold, and $\eta<0$. The solution of equation (11) is unstable for $\bar{\delta} \geq \bar{\delta}_{S N}$.

Proof. For $N \geq 2$, because $T_{2 N}$ is a symmetric matrix, the minimum eigenvalue $\lambda_{s}$ and the maximum eigenvalue $\lambda_{l}$ satisfy $\lambda_{s} \leq\left\langle\tilde{\boldsymbol{x}}, T_{2 N} \tilde{\boldsymbol{x}}\right\rangle /\|\tilde{\boldsymbol{x}}\|^{2} \leq \lambda_{l}$. For the upper branch, $b_{1}=b_{1}^{+}$is negative by Lemma 1 . Thus, by taking $\tilde{\boldsymbol{x}}={ }^{t}\left(\tilde{x}_{0}, \ldots, \tilde{x}_{2 N-1}\right)={ }^{t}(0,1, \ldots, 1,0)$, we obtain

$$
\begin{aligned}
\left\langle\tilde{\boldsymbol{x}}, T_{2 N} \tilde{\boldsymbol{x}}\right\rangle= & -b_{0}\left[2 \tilde{x}_{0}^{2}+\left(\tilde{x}_{1}-\tilde{x}_{2}\right)^{2}+\cdots+\left(\tilde{x}_{2 N-3}-\tilde{x}_{2 N-2}\right)^{2}+2 \tilde{x}_{2 N-1}^{2}\right] \\
& -b_{1}\left[\left(\tilde{x}_{0}-\tilde{x}_{1}\right)^{2}+\cdots+\left(\tilde{x}_{2 N-2}-\tilde{x}_{2 N-1}\right)^{2}\right] \\
= & -2 b_{1}>0
\end{aligned}
$$

which shows that the maximum eigenvalue is positive.

Thus, Theorem 2 is proved.

\section{Proof of Theorem 3}

Let $U_{2 N}(\bar{\delta})$ be a $2 N \times 2 N$ linearized matrix of (A.23) with periodic boundary conditions for $y_{j}=\bar{q}(\bar{\delta})+\bar{r}(\bar{\delta})=\bar{r}(\bar{\delta})$ and $z_{j}=\bar{q}(\bar{\delta})-\bar{r}(\bar{\delta})=-\bar{r}(\bar{\delta})$. That is,

$$
U_{2 N}(\bar{\delta})=\left(\begin{array}{ccccccc}
a & b_{1} & & & & & b_{0} \\
b_{1} & a & b_{0} & & & & \\
& b_{0} & a & b_{1} & & & \\
& & \ddots & \ddots & \ddots & & \\
& & & b_{1} & a & b_{0} & \\
& & & & b_{0} & a & b_{1} \\
b_{0} & & & & & b_{1} & a
\end{array}\right) .
$$

The eigenvalues of $U_{2 N}(\bar{\delta})$ can be calculated using the following lemma. 
Lemma 9. Let $U_{N}^{+}(\bar{\delta})$ and $U_{N}^{-}(\bar{\delta})$ be $N \times N$ matrices such that

$$
\begin{aligned}
& U_{N}^{ \pm}(\bar{\delta}):=\left(\begin{array}{ccccccc}
a \pm b_{0} & b_{1} & & & & & \\
b_{1} & a & b_{0} & & & & \\
& b_{0} & a & b_{1} & & & \\
& & \ddots & \ddots & \ddots & & \\
& & & b_{1} & a & b_{0} & \\
& & & & b_{0} & a & b_{1} \\
& & & & & b_{1} & a \pm b_{0}
\end{array}\right) \quad(N: \text { even }) \\
& U_{N}^{+}(\bar{\delta}):=\left(\begin{array}{ccccccc}
a+b_{0} & b_{1} & & & & & \\
b_{1} & a & b_{0} & & & & \\
& b_{0} & a & b_{1} & & & \\
& & \ddots & \ddots & \ddots & & \\
& & & b_{0} & a & b_{1} & \\
& & & & b_{1} & a & b_{0} \\
& & & & & b_{0} & a+b_{1}
\end{array}\right) \\
& U_{N}^{-}(\bar{\delta}):=\left(\begin{array}{ccccccc}
a-b_{1} & b_{0} & & & & & \\
b_{0} & a & b_{1} & & & & \\
& b_{1} & a & b_{0} & & & \\
& & \ddots & \ddots & \ddots & & \\
& & & b_{1} & a & b_{0} & \\
& & & & b_{0} & a & b_{1} \\
& & & & & b_{1} & a-b_{0}
\end{array}\right) \quad \text { (N: odd). }
\end{aligned}
$$

The eigenvalues of $U_{2 N}$ are calculated by solving the equation

$$
\left|U_{N}^{+}-\lambda I_{N}\right|\left|U_{N}^{-}-\lambda I_{N}\right|=0,
$$

where $I_{N}$ is the $N \times N$ identity matrix.

Proof.

$$
\begin{aligned}
\left|U_{2 N}(\bar{\delta})-\lambda I_{2 N}\right| & =\left|\begin{array}{cc}
U_{N}^{\prime}(\bar{\delta})-\lambda I_{N} & B_{N}^{\prime}(\bar{\delta}) \\
B_{N}^{\prime \prime}(\bar{\delta}) & U_{N}^{\prime \prime}(\bar{\delta})-\lambda I_{N}
\end{array}\right| \\
& =\left|U_{N}^{\prime}(\bar{\delta})+\widetilde{B}_{N}^{\prime}(\bar{\delta})-\lambda I_{N}\right|\left|U_{N}^{\prime \prime}(\bar{\delta})-\widetilde{B}_{N}^{\prime \prime}(\bar{\delta})-\lambda I_{N}\right| \\
& =\left|U_{N}^{+}(\bar{\delta})-\lambda I_{N}\right|\left|U_{N}^{-}(\bar{\delta})-\lambda I_{N}\right|,
\end{aligned}
$$


where $U_{N}^{\prime}, U_{N}^{\prime \prime}, B_{N}^{\prime}, B_{N}^{\prime \prime}, \widetilde{B_{N}^{\prime}}$, and $\widetilde{B_{N}^{\prime \prime}}$ are defined as follows.

Case I) $N$ is even.

$$
\begin{aligned}
& U_{N}^{\prime}(\bar{\delta})=U_{N}^{\prime \prime}(\bar{\delta}):=\left(\begin{array}{ccccccc}
a & b_{1} & & & & & \\
b_{1} & a & b_{0} & & & & \\
& b_{0} & a & b_{1} & & & \\
& & \ddots & \ddots & \ddots & & \\
& & & b_{1} & a & b_{0} & \\
& & & & b_{0} & a & b_{1} \\
& & & & & b_{1} & a
\end{array}\right), \\
& B_{N}^{\prime}(\bar{\delta})=B_{N}^{\prime \prime}(\bar{\delta}):=\left(\begin{array}{ccccccc} 
& & & & & 0 & b_{0} \\
& & & & 0 & 0 & 0 \\
& & & 0 & 0 & 0 & \\
& & . & . & . & & \\
& 0 & 0 & 0 & & & \\
0 & 0 & 0 & & & & \\
b_{0} & 0 & & & & &
\end{array}\right), \\
& \widetilde{B}_{N}^{\prime}(\bar{\delta})=\widetilde{B}_{N}^{\prime \prime}(\bar{\delta}):=\left(\begin{array}{ccccccc}
b_{0} & 0 & & & & & \\
0 & 0 & 0 & & & & \\
& 0 & 0 & 0 & & & \\
& & \ddots & \ddots & \ddots & & \\
& & & 0 & 0 & 0 & \\
& & & & 0 & 0 & 0 \\
& & & & & 0 & b_{0}
\end{array}\right),
\end{aligned}
$$


Case II) $N$ is odd.

$$
\begin{aligned}
& U_{N}^{\prime}(\bar{\delta}):=\left(\begin{array}{ccccccc}
a & b_{1} & & & & & \\
b_{1} & a & b_{0} & & & & \\
& b_{0} & a & b_{1} & & & \\
& & \ddots & \ddots & \ddots & & \\
& & & b_{0} & a & b_{1} & \\
& & & & b_{1} & a & b_{0} \\
& & & & & b_{0} & a
\end{array}\right), \quad U_{N}^{\prime \prime}(\bar{\delta}):=\left(\begin{array}{ccccccc}
a & b_{0} & & & & & \\
b_{0} & a & b_{1} & & & & \\
& b_{1} & a & b_{0} & & & \\
& & \ddots & \ddots & \ddots & & \\
& & & b_{1} & a & b_{0} & \\
& & & & b_{0} & a & b_{1} \\
& & & & & b_{1} & a
\end{array}\right),
\end{aligned}
$$

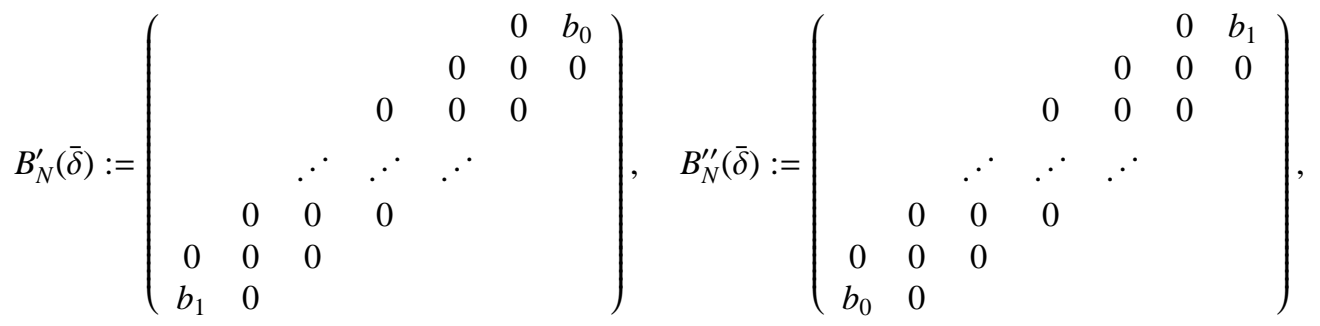

$$
\begin{aligned}
& \widetilde{B}_{N}^{\prime}(\bar{\delta}):=\left(\begin{array}{ccccccc}
b_{0} & 0 & & & & & \\
0 & 0 & 0 & & & & \\
& 0 & 0 & 0 & & & \\
& & \ddots & \ddots & \ddots & & \\
& & & 0 & 0 & 0 & \\
& & & & 0 & 0 & 0 \\
& & & & & 0 & b_{1}
\end{array}\right), \quad \widetilde{B}_{N}^{\prime \prime}(\bar{\delta}):=\left(\begin{array}{ccccccc}
b_{1} & 0 & & & & & \\
0 & 0 & 0 & & & & \\
& 0 & 0 & 0 & & & \\
& & \ddots & \ddots & \ddots & & \\
& & & 0 & 0 & 0 & \\
& & & 0 & 0 & 0 \\
& & & & & 0 & b_{0}
\end{array}\right) .
\end{aligned}
$$

In order to obtain (A.39), we add the $(2 N-n+1)$-th column of (A.38) to the $n$-th column of (A.38) for $n=1, \ldots, N$. Then we subtract the $n$-th row of (A.38) from the $(2 N-n+1)$-th row of (A.38) for $n=1, \ldots, N$.

First, we perform a linear stability analysis of the solutions of the lower branch. The stability of 1-stripe solutions can be proved in a similar way to Lemma 1. Thus, we omit proofs of Lemmas 10 and 11.

Lemma 10. The eigenvalues of $U_{2}$ corresponding to the lower branch are 0 and $2 a^{-}$.

From Lemmas 1 and 10, it is proved that, for $N=1$, the eigenvalues of the lower branch consist of one zero eigenvalue and one negative eigenvalue.

The number of zero eigenvalues corresponding to the lower branch for $N \geq 2$ is given by the following Lemma.

Lemma 11. If $N \geq 2, U_{2 N}$ corresponding to the lower branch has zero eigenvalues of multiplicity $N$ at $\bar{\delta}=\bar{\delta}_{B P}^{-}$, zero eigenvalues of multiplicity 2 at $\bar{\delta}=\bar{\delta}_{S N}$, and one zero eigenvalue for $\bar{\delta} \in\left(\bar{\delta}_{S N}, \bar{\delta}_{B P}^{-}\right) \cup\left(\bar{\delta}_{B P}^{-}, \bar{\delta}_{B P}^{+}\right)$

From Lemma 11, we see that $\lambda=2 a(\bar{\delta})$ is the eigenvalue of $U_{2 N}$ corresponding to the saddlenode bifurcation because $a(\bar{\delta})=0$ for $\bar{\delta}=\bar{\delta}_{S N}$ from Lemma 1 .

Next, we consider the number of positive eigenvalues of $P_{n}$ for the lower branch. 
Lemma 12. If $N \geq 2, U_{2 N}$ corresponding to the lower branch has $N-1$ positive eigenvalues for $\bar{\delta} \in\left[\bar{\delta}_{S N}, \bar{\delta}_{B P}^{-}\right)$and no positive eigenvalues for $\bar{\delta} \in\left[\bar{\delta}_{B P}^{-}, \bar{\delta}_{B P}^{+}\right)$.

Proof. $\quad U_{2 N}$ is a symmetric matrix and

$$
\begin{aligned}
\left\langle\tilde{\boldsymbol{x}}, U_{2 N} \tilde{\boldsymbol{x}}\right\rangle /\|\tilde{\boldsymbol{x}}\|^{2}= & {\left[-b_{0}\left(\left(\tilde{x}_{1}-\tilde{x}_{2}\right)^{2}+\left(\tilde{x}_{3}-\tilde{x}_{4}\right)^{2}+\cdots+\left(\tilde{x}_{2 N-1}-\tilde{x}_{0}\right)^{2}\right)\right.} \\
& \left.-b_{1}\left(\left(\tilde{x}_{0}-\tilde{x}_{1}\right)^{2}+\left(\tilde{x}_{2}-\tilde{x}_{3}\right)^{2}+\cdots+\left(\tilde{x}_{2 N-2}-\tilde{x}_{2 N-1}\right)^{2}\right)\right] /\|\tilde{\boldsymbol{x}}\|^{2}
\end{aligned}
$$

holds for $\tilde{\boldsymbol{x}}={ }^{t}\left(\tilde{x}_{0}, \tilde{x}_{1}, \ldots, \tilde{x}_{2 N-1}\right)$. Because $b_{0}$ and $b_{1}^{-}$are positive by Lemma $1,\left\langle\tilde{\boldsymbol{x}}, U_{2 N} \tilde{\boldsymbol{x}}\right\rangle \leq$ 0 holds for $\bar{\delta} \in\left[\bar{\delta}_{B P}^{-}, \bar{\delta}_{B P}^{+}\right)$. Therefore, $U_{2 N}$ with respect to $\left(q_{j}, r_{j}\right)=\left(0, \bar{r}_{-}\right)$has no positive eigenvalue for $\bar{\delta} \in\left[\bar{\delta}_{B P}^{-}, \bar{\delta}_{B P}^{+}\right.$).

In a manner similar to the proof of Lemma 4, we can prove the following. If $N$ is even, $U_{2 N}^{+}$ has $N / 2-1$ positive eigenvalues and $U_{2 N}^{-}$has $N / 2$ positive eigenvalues for $\bar{\delta}=\bar{\delta}_{S N}$; if $N$ is odd, $U_{N}^{+}$and $U_{N}^{-}$have $(N-1) / 2$ positive eigenvalues for $\bar{\delta}=\bar{\delta}_{S N}$. Therefore, $U_{2 N}$ has $N-1$ positive eigenvalues for $\bar{\delta}=\bar{\delta}_{S N}$. Because $U_{2 N}$ has no zero eigenvalue except for that arising from rotational invariance, $U_{2 N}$ has $N-1$ positive eigenvalues for $\bar{\delta} \in\left[\bar{\delta}_{S N}, \bar{\delta}_{B P}^{-}\right)$.

Lemma 13. Assume that $N \geq 2$ and $\bar{\delta} \in\left[\bar{\delta}_{S N}, \bar{\delta}_{B P}^{-}\right)$. If $N$ is even, the positive eigenvalues of $U_{2 N}$ corresponding to the lower branch consist of one distinct maximum eigenvalue and $(N-2) / 2$ pairs of distinct eigenvalues. If $N$ is odd, the positive eigenvalues of $U_{2 N}$ corresponding to the lower branch consist of $(N-1) / 2$ pairs of distinct eigenvalues.

Proof. From Lemma 1 and Theorem 4, it is apparent that the symmetric tri-diagonal matrices $U_{N}^{+}(\bar{\delta})$ and $U_{N}^{-}(\bar{\delta})$ have distinct eigenvalues. Therefore, from Lemma 9, the multiplicity of positive eigenvalues of $U_{2 N}(\bar{\delta})$ for $\bar{\delta} \in\left[\bar{\delta}_{S N}, \bar{\delta}_{B P}^{-}\right)$is at most 2.

Case I) $N$ is even.

Let $\varphi_{i}^{+}:={ }^{t}\left(y_{0}^{i}, z_{0}^{i}, y_{1}^{i}, z_{1}^{i}, \ldots, y_{N-1}^{i}, z_{N-1}^{i}\right)$ be eigenvectors corresponding to the eigenvalue $\tilde{\lambda}_{i}$ of $U_{N}^{+}(\bar{\delta})$. We define

$$
\widetilde{\varphi}_{i}^{+}:={ }^{t}\left(y_{N-1}^{i}, z_{N-1}^{i}, y_{N-2}^{i}, z_{N-2}^{i}, \ldots, y_{0}^{i}, z_{0}^{i}\right) .
$$

Then,

$$
\boldsymbol{\varphi}_{i}:={ }^{t}\left({ }^{t} \boldsymbol{\varphi}_{i}^{+},{ }^{t} \widetilde{\boldsymbol{\varphi}}_{i}^{+}\right) \quad(i=0, \ldots, N-1)
$$

are eigenvectors of $U_{2 N}(\bar{\delta})$ corresponding to $\tilde{\lambda}_{i}$.

From the condition of rotational invariance, $\varphi_{i}^{\prime}={ }^{t}\left(y_{N-1}^{i}, z_{N-1}^{i}, y_{0}^{i}, z_{0}^{i}, \ldots, y_{N-2}^{i}, z_{N-2}^{i}\right)$ is an eigenvector of $U_{2 N}$ corresponding to $\tilde{\lambda}_{i}$. If $\boldsymbol{\varphi}_{i}$ and $\boldsymbol{\varphi}_{i}^{\prime}$ are linearly dependent, i.e., $\tilde{a} \boldsymbol{\varphi}_{i}+\tilde{b} \boldsymbol{\varphi}_{i}^{\prime}=0$ for $\tilde{a} \neq 0$ and $\tilde{b} \neq 0$, then $(-\tilde{a} / \tilde{b})^{N}=1$ holds. We define

$$
\begin{array}{ll}
\boldsymbol{\varphi}_{0}:={ }^{t}\left(y_{0}^{0}, z_{0}^{0}, y_{1}^{0}, z_{1}^{0}, \ldots, y_{N-1}^{0}, z_{N-1}^{0}\right), & y_{j}^{0}=-1, z_{j}^{0}=-1, \\
\boldsymbol{\varphi}_{1}:={ }^{t}\left(y_{0}^{1}, z_{0}^{1}, y_{1}^{1}, z_{1}^{1}, \ldots, y_{N-1}^{1}, z_{N-1}^{1}\right), & y_{j}^{1}=(-1)^{j+1}, z_{j}^{1}=(-1)^{j}, \\
\boldsymbol{\varphi}_{2}:={ }^{t}\left(y_{0}^{2}, z_{0}^{2}, y_{1}^{2}, z_{1}^{2}, \ldots, y_{N-1}^{2}, z_{N-1}^{2}\right), & y_{j}^{2}=(-1)^{j+1}, z_{j}^{2}=(-1)^{j+1}, \\
\boldsymbol{\varphi}_{3}:={ }^{t}\left(y_{0}^{3}, z_{0}^{3}, y_{1}^{3}, z_{1}^{3}, \ldots, y_{N-1}^{3}, z_{N-1}^{3}\right), & y_{j}^{3}=-1, z_{j}^{3}=1 .
\end{array}
$$

Now, $\varphi_{j}(j=0,1,2,3)$ are eigenvectors corresponding to the eigenvalues $\tilde{\lambda}_{0}=0, \tilde{\lambda}_{1}=-2 b_{1}^{-}$, $\tilde{\lambda}_{2}=-2 b_{0}$, and $\tilde{\lambda}_{3}=2 a^{-}$. From Lemma $1, \tilde{\lambda}_{1}>0, \tilde{\lambda}_{2}<0$, and $\tilde{\lambda}_{3}<0$ for $\bar{\delta} \in\left(\bar{\delta}_{S N}, \bar{\delta}_{B P}^{-}\right)$. From Theorem 4, $\tilde{\lambda}_{i}(i=0,1,2,3)$ are distinct for $\bar{\delta} \in\left(\bar{\delta}_{S N}, \bar{\delta}_{B P}^{-}\right)$. In addition, $\varphi_{j}$ and $\varphi_{j}^{\prime}$ are 
linearly independent except for the above four eigenvectors, and they have the same eigenvalue. Therefore, the positive eigenvalues for $\bar{\delta} \in\left(\bar{\delta}_{S N}, \bar{\delta}_{B P}\right)$ consist of $(N-2) / 2$ pairs of distinct eigenvalues and one distinct eigenvalue. In addition, $\tilde{\lambda}_{1}$ is the maximum eigenvalue for $\bar{\delta} \in$ $\left[\bar{\delta}_{S N}, \bar{\delta}_{B P}^{-}\right.$), because $\left\langle\tilde{\boldsymbol{x}}, U_{2 N} \tilde{\boldsymbol{x}}\right\rangle /\|\tilde{\boldsymbol{x}}\|^{2}$ is maximum for $\tilde{\boldsymbol{x}}=\boldsymbol{\varphi}_{1}$.

\section{Case II) $N$ is odd.}

Let $\varphi_{i}^{+}:={ }^{t}\left(y_{0}^{i}, z_{0}^{i}, y_{1}^{i}, z_{1}^{i}, \ldots, y_{(N-1) / 2-1}^{i}, z_{(N-1) / 2-1}^{i} y_{(N-1) / 2}^{i}\right)$ be an eigenvector corresponding to the eigenvalue $\tilde{\lambda}_{i}$ of $U_{N}^{+}$, and define $\widetilde{\varphi}_{i}^{+}:={ }^{t}\left(y_{(N-1) / 2}^{i}, z_{(N-1) / 2-1)}^{i}, y_{(N-1) / 2-1}^{i}, \ldots, z_{1}^{i}, y_{1}^{i}, z_{0}^{i}, y_{0}^{i}\right)$. Then $\boldsymbol{\varphi}_{i}:={ }^{t}\left({ }^{t} \boldsymbol{\varphi}_{i}^{+},{ }^{\boldsymbol{\varphi}_{i}^{+}}\right)$is an eigenvector corresponding to $\tilde{\lambda}_{i}$. If $\boldsymbol{\varphi}_{i}$ and $\boldsymbol{\varphi}_{i}^{\prime}={ }^{t}\left(y_{N-1}^{i}, z_{N-1}^{i}, y_{0}^{i}, z_{0}^{i}, \ldots, y_{N-2}^{i}, z_{N-2}^{i}\right)$ are linearly dependent, i.e., $\tilde{a} \varphi_{i}+\tilde{b} \varphi_{i}^{\prime}=0$ for $\tilde{a} \neq 0$ and $\tilde{b} \neq 0$, then $(-\tilde{a} / \tilde{b})^{N}=1$ holds. Thus we define

$$
\begin{array}{lll}
\varphi_{0}:={ }^{t}\left(y_{0}^{0}, z_{0}^{0}, y_{1}^{0}, z_{1}^{0}, \ldots, y_{N-1}^{0}, z_{N-1}^{0}\right), & y_{j}^{0}=-1, z_{j}^{0}=-1, \\
\varphi_{1}:=\quad{ }^{t}\left(y_{0}^{1}, z_{0}^{1}, y_{1}^{1}, z_{1}^{1}, \ldots, y_{N-1}^{1}, z_{N-1}^{1}\right), & y_{j}^{1}=-1, z_{j}^{1}=1 .
\end{array}
$$

Now, $\varphi_{j}(j=0,1)$ are eigenvectors corresponding to $\tilde{\lambda}_{j}$, where $\tilde{\lambda}_{0}=0$ and $\tilde{\lambda}_{1}=2 a^{-}<0$. By a similar argument to that used in the case where $N$ is even, we can conclude that the positive eigenvalues of $U_{2 N}$ consist of $(N-1) / 2$ pairs.

We obtain the following lemma directly from the rotational invariance of the $N$-stripe solutions.

Lemma 14. If ${ }^{t}\left(q_{0}^{i}, q_{1}^{i}, \ldots, q_{N-1}^{i}, r_{0}^{i}, r_{1}^{i}, \ldots, r_{N-1}^{i}\right)$ is an eigenvector of (10) with periodic boundary conditions, then the index-rotated eigenvector ${ }^{t}\left(q_{N-1}^{i}, q_{0}^{i}, q_{1}^{i} \ldots, q_{N-2}^{i}, r_{N-1}^{i}, r_{0}^{i}, r_{1}^{i}, \ldots, r_{N-1}^{i}\right)$ is an eigenvector of (10).

The signs of $r_{j}^{l}$ and $r_{j+1}^{l}$ are given by the following lemma.

Lemma 15. If $N$ is even, $r_{j}^{l}$ and $r_{j+1}^{l}$ have different signs for $\bar{\delta} \in\left[\bar{\delta}_{S N}, \bar{\delta}_{B P}^{-}\right)$. If $N$ is odd, there exist two linearly independent eigenfunctions corresponding to the maximum eigenvalues, which are given by

$$
\left(0, r_{1}^{l}, r_{2}^{l}, \ldots, r_{N}^{l}\right)
$$

and

$$
\left(r_{N}^{l}, 0, r_{1}^{l}, r_{2}^{l}, \ldots, r_{N-1}^{l}\right),
$$

where $r_{j}^{l}$ and $r_{j+1}^{l}(j=1,2, \ldots, N-1)$ have different signs.

Proof.

Case I) $N$ is even.

As shown in the proof of Lemma 13, $\tilde{\lambda}_{1}$ is the maximum eigenvalue and $\varphi_{1}$ is given by (A.40). Therefore $r_{j}^{l}$ and $r_{j+1}^{l}$ have different signs for $j=0,1, \ldots, N-2$, and $r_{0}^{l}$ and $r_{N-1}^{l}$ have different signs.

Case II) $N$ is odd.

From Lemma 13, there exist two maximum eigenvalues. From Lemma 4, the eigenvectors corresponding to the maximum eigenvalue $\tilde{\lambda}_{l}$ of $U_{2 N}$ for $\left(q_{j}, r_{j}\right)=\left(0, \bar{r}_{-}\right)$satisfy the following 
conditions:

$$
\begin{aligned}
& y_{j}^{l}=\frac{p_{j}^{+}\left(\tilde{\lambda}_{l}\right)}{\left(b_{0}\right)^{j}\left(b_{1}^{-}\right)^{j}}, z_{j}^{i}=\frac{-p_{j+1}^{+}\left(\tilde{\lambda}_{l}\right)}{\left(b_{0}\right)^{j}\left(b_{1}^{-}\right)^{j+1}}, \quad(j=0,1, \ldots,(N-1) / 2-1), \\
& y_{j}^{i}=z_{j}^{i}=\frac{p_{j}^{+}\left(\tilde{\lambda}_{l}\right)}{\left(b_{0}\right)^{j}\left(b_{1}^{-}\right)^{j}}, \quad(j=(N-1) / 2), \\
& y_{j}^{i}=z_{N-j-1}^{i}, z_{j}^{i}=y_{N-j-1}^{i}, \quad(j=(N-1) / 2+1,(N-1) / 2+2, \ldots, N-1) .
\end{aligned}
$$

From Theorem 5, $p_{j}^{+}\left(\tilde{\lambda}_{l}\right)$ and $p_{j}^{-}\left(\tilde{\lambda}_{l}\right)$ have different signs for $\bar{\delta} \in\left[\bar{\delta}_{S N}, \bar{\delta}_{B P}^{-}\right)$because, by Lemma 1 , $b_{1}^{-}$is negative for $\bar{\delta} \in\left[\bar{\delta}_{S N}, \bar{\delta}_{B P}^{-}\right)$. Therefore, by using Lemma 14 inductively, Lemma 15 holds.

The properties of the eigenvectors are obtained in a similar way in the case of Neumann boundary conditions.

Lemma 16. The eigenfunction of $U_{2 N}$ corresponding to eigenvalue $\tilde{\lambda}_{s}=2 a^{-}$is given by $q_{j}^{s}=$ $0, r_{j}^{s}=1$.

The following lemma can be proved in a parallel manner to the proof of Lemma 7. Therefore, we omit it here.

Lemma 17. Let $\tilde{\boldsymbol{x}}=\left(\tilde{x}_{0}, \tilde{x}_{1}, \ldots, \tilde{x}_{2 N-1}\right)$ be an eigenvector of $U_{2 N}$ corresponding to an eigenvalue $\tilde{\lambda} \neq 2 a$. Then $\tilde{\boldsymbol{x}}$ and $\left(\bar{y}_{0}, \bar{z}_{0}, \bar{y}_{1}, \bar{z}_{1}, \ldots, \bar{y}_{N-1}, \bar{z}_{N-1}\right)$ are orthogonal.

Finally, we have the following Lemma 18 concerning the stability of the upper branch.

Lemma 18. The stationary solution (11) is unstable for $\bar{\delta} \geq \bar{\delta}_{S N}$.

Proof. Because $U_{2 N}$ is a symmetric matrix, the maximum eigenvalue $\tilde{\lambda}_{l}$ is bounded: $\left\langle\tilde{\boldsymbol{x}}, U_{2 N} \tilde{\boldsymbol{x}}\right\rangle /\|\tilde{\boldsymbol{x}}\|^{2} \leq$ $\tilde{\lambda}_{l}$, where

$$
\begin{aligned}
\left\langle\tilde{\boldsymbol{x}}, U_{2 N} \tilde{\boldsymbol{x}}\right\rangle /\|\tilde{\boldsymbol{x}}\|^{2}= & {\left[-b_{0}\left(\left(\tilde{x}_{1}-\tilde{x}_{2}\right)^{2}+\left(\tilde{x}_{3}-\tilde{x}_{4}\right)^{2}+\cdots+\left(\tilde{x}_{2 N-1}-\tilde{x}_{0}\right)^{2}\right)\right.} \\
& \left.-b_{1}\left(\left(\tilde{x}_{0}-\tilde{x}_{1}\right)^{2}+\left(\tilde{x}_{2}-\tilde{x}_{3}\right)^{2}+\cdots+\left(\tilde{x}_{2 N-2}-\tilde{x}_{2 N-1}\right)^{2}\right)\right] /\|\tilde{\boldsymbol{x}}\|^{2},
\end{aligned}
$$

for $\tilde{\boldsymbol{x}}={ }^{t}\left(\tilde{x}_{0}, \tilde{x}_{1}, \ldots, \tilde{x}_{2 N-1}\right)$. Because $b_{1}^{+}$is negative for $\bar{\delta} \geq \bar{\delta}_{S N}$ by Lemma $1,\left\langle\tilde{\boldsymbol{x}}, U_{2 N} \tilde{\boldsymbol{x}}\right\rangle$ attains a positive value for $\tilde{\boldsymbol{x}}={ }^{t}(-1,1,1,-1, \ldots)$, which shows that the maximum eigenvalue is positive. Therefore, the upper branch is unstable for $\bar{\delta} \geq \bar{\delta}_{S N}$.

Theorem 3 follows from Lemmas 10 to 18 .

\section{References}

[1] S. Kondo, R. Asai, A reaction-diffusion wave on the skin of the marine angelfish Pomacanthus, Nature 376 (1995) 765-768.

[2] A. M. Turing, The chemical basis of morphogenesis, Phil. Trans. R. Soc. B 237 (1952) 37-72.

[3] T. Höfer and P. K. Maini, Turing patterns in fish skin?, Nature 380 (1996) 678.

[4] P. M. Kulesa et al., On a model mechanism for the spatial patterning of teeth primordia in the Alligator, J. Theor. Biol. 180 (1996) 287-296.

[5] M. Hirata, K. Nakamura, T. Kanemaru, Y. Shibata, S. Kondo, Pigment cell organization in the hypodermis of zebrafish, Dev. Dynam. 227 (2003) 497-503.

[6] P. T. Saunders, M. W. Ho, Reliable segmentation by successive bifurcation, Bull. Math. Biol. 57 (1995) $539-556$. 
[7] E. J. Crampin, E. A. Gaffney, P. K. Maini, Reaction and diffusion on growing domains: Scenarios for robust pattern formation, Bull. Math. Biol. 61 (1999) 1093-1120.

[8] E. J. Crampin, E. A. Gaffney, P. K. Maini, Mode-doubling and tripling in reaction-diffusion patterns on growing domains: a piecewise linear model, J. Math. Biol. 44 (2002) 107-128.

[9] E. J. Crampin, W. W. Hackborn, P. K. Maini, Pattern formation in reaction-diffusion models with nonuniform growth, Bull. Math. Biol., 64 (2002) 747-769.

[10] R. G. Plaza et al., The effect of growth and curvature on pattern formation, J. Dyn. Differ. Equ. 16 (2004) 1093 1121.

[11] A. A. Neville, P. C. Matthews, H. M. Byrne, Interactions between pattern formation and domain growth, Bull. Math. Biol. 68 (2006) 1975-2003.

[12] T. Miura, K. Shiota, G. Morriss-Kay, P. K. Maini, Mixed mode pattern in Doublefoot mutant mouse limb - Turing reaction-diffusion model on a growing domain during limb development, J. Theor. Biol. 240 (2006) 562-573.

[13] K. J. Painter, P. K. Maini, H. G. Othmer, Stripe formation in juvenile Pomacanthus explained by a generalized Turing mechanism with chemotaxis, Proc. Natl. Acad. Sci. USA 96 (1999) 5549-5554.

[14] C. Varea, J. L. Argón, R. A. Barrio, Confined Turing patterns in growing systems, Phys. Rev. E 56 (1997) 12501253.

[15] P. Gray, S. K. Scott, Autocatalytic reactions in the isothermal, continuous stirred tank reactor: oscillations and instabilities in the system A $+2 B \rightarrow 3 B, B \rightarrow$ C, Chem. Eng. Sci. 39 (1984) 1087-1097.

[16] J. E. Pearson, Complex patterns in a simple system, Science 216 (1993) 189-192.

[17] A. Madzvamuse, R. D. K. Thomas, P. K. Maini, A. J. Wathen, A numerical approach to the study of spatial pattern formation in the ligaments of arcoid bivalves, Bull. Math. Biol. 64 (2002) 501-530.

[18] I. Barrass, E. J. Crampin, P. K. Maini, Mode transitions in a model reaction-diffusion system driven by domain growth and noise, Bull. Math. Biol. 68 (2006) 981-995.

[19] A. Doelman, W. Eckhaus, T. J. Kaper, Slowly-modulated two-pulse solutions in the Gray-Scott model I: Asymptotic construction and stability, SIAM J. Appl. Math. 61 (2000) 1080-1102.

[20] A. Doelman, T. J. Kaper, P. Zegeling, Pattern formation in the one-dimensional Gray-Scott model, Nonlinearity 10 (1997) 523-563.

[21] A. Doelman, R. A. Gardner, T. J. Kaper, Stability analysis of singular patterns in the 1D Gray-Scott model: a matched asymptotics approach, Physica D 122 (1998) 1-36.

[22] A. Doelman, W. Eckhaus, T. J. Kaper, Slowly modulated two-pulse solutions in the Gray-Scott model II: geometric theory, bifurcations, and splitting dynamics, SIAM J. Appl. Math. 61 (2001) 2036-2062.

[23] A. Doelman, R. A. Gardner, T. J. Kaper, Large stable pulse solutions in reaction-diffusion equations, Indiana Univ. Math. J. 50 (2001) 443-507.

[24] A. Doelman, R. A. Gardner, T. J. Kaper, A stability index analysis of 1-D patterns of the Gray-Scott model, Mem. Amer. Math. Soc. 155 (2002) no. 737

[25] T. Kolokolnikov, M. J. Ward, J. Wei, The existence and stability of spike equilibria in the one-dimensional GrayScott model: The pulse-splitting regime, Physica D 202 (2005) 258-293.

[26] M. J. Ward, J. Wei, The existence and stability of asymmetric spike patterns in the Schnakenburg model, Studies in Appl. Math. 109 (2002) 229-264.

[27] C. Muratov, V. V. Osipov, Traveling spike auto-solitons in the Gray-Scott model, Physica D 155 (2001) $112-131$.

[28] C. Muratov, V. V. Osipov, Stability of the static spike autosolitons in the Gray-Scott model, SIAM J. Appl. Math. 62 (2002) 1463-1487.

[29] C. Muratov, V. V. Osipov, Static spike autosolitons in the Gray-Scott model, J. Phys. A 33 (2000) 8893-8916.

[30] S. Zelik, A. Mielke, Multi-pulse evolution and space-time chaos in dissipative systems, Mem. Amer. Math. Soc. 198 (2009) no. 925.

[31] Y. Nishiura, D. Ueyama, A skeleton structure of self-replicating dynamics, Physica D, 130 (1999) 73-104.

[32] S. -I. Ei, Y. Nishiura, K. -I. Ueda, $2^{n}$-splitting or edge-splitting? - A Manner of Splitting in Dissipative Systems, Japan J. Ind. Appl. Math. 18 (2001) 181-205.

[33] V. Petrov, S. K. Scott, K. Showalter, Excitability, wave reflection, and wave splitting in a cubic autocatalysis reaction-diffusion system, Phil. Trans. R. Soc. Lond. A 347 (1994) 631-642.

[34] P. De Kepper, J. J. Perraud, B. Rudovics, E. Dulos, Experimental study of stationary Turing patterns and their interaction with traveling waves in a chemical system, Int. J. Bifurcation and Chaos 4 (1994) 1215-1231.

[35] William H. Press, B. P. Flannery, S. A. Teukolsky, S. A. Teukolsky, Numerical Recipes in C: The Art of Scientific Computing, second ed., Cambridge University Press, Cambridge, 1992.

[36] K. J. Lee, W. D. McCormick, J. E. Pearson, H. L. Swinney, Experimental observation of self-replicating spots in a reaction- diffusion system, Nature 369 (1994) 215-218.

[37] H. Fujii, M. Mimura, Y. Nishiura, A picture of the global bifurcation diagram in ecological interacting and diffusing systems, Physica D 5 (1982) 1-42.

[38] S. -I. Ei, The motion of weakly interacting pulses in reaction-diffusion systems, J. Dyn. Differ. Equ. 14 (2002) 
$85-137$.

[39] M. Golubitsky, I. Stewart, The Symmetry Perspective: From Equilibrium to Chaos in Phase Space and Physical Space, Birkhauser Verlag, 2003.

[40] A. Gierer, H. Meinhardt, A theory of biological pattern formation, Kybernetik 12 (1972) 30-39.

[41] D. Henry, Geometric Theory of Semilinear Parabolic Equations. Series: Lecture Notes in Mathematics, Vol. 840, Springer-Verlag, 1981.

[42] J. H. Wilkinson, The Algebraic Eigenvalue Problem, Clarendon Press, Oxford, 1965. 